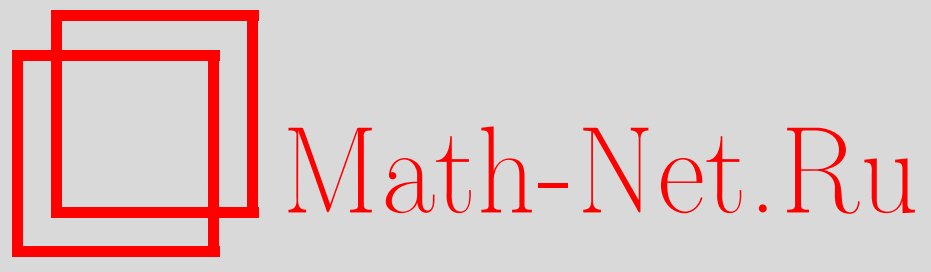

Н. Г. Мощевитин, Сингулярные диофантовы системы А. Я. Хинчина и их применение, УМH, 2010, том 65, выпуск $3,43-126$

DOI: https://doi.org/10.4213/rm9354

Использование Общероссийского математического портала Math-Net.Ru подразумевает, что вы прочитали и согласны с пользовательским соглашением http://www . mathnet.ru/rus/agreement

Параметры загрузки:

IP: 18.234 .197 .8

26 апреля 2023 г., 12:50:53

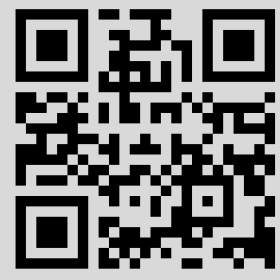


Моему замечательному учителю Валерию Васильевичу Козлову к его шестидесятилетию

\section{Сингулярные диофантовы системы А. Я. Хинчина и их применение}

\section{Н. Г. Мощевитин}

Работа представляет собой обзор классических и новых методов из теории диофантовых приближений и ее приложений, так или иначе связанных с применением результатов А.Я. Хинчина о существовании вещественных чисел, допускающих аномально хорошие приближения рациональными числами.

Библиография: 145 названий.

Ключевые слова: многомерные диофантовы приближения, цепные дроби, сингулярные системы Хинчина, наилучшие приближения, диофантовы неравенства, теоремы переноса, задача Козлова, метод ПересаШлага.

\section{СОДЕРЖАНИЕ}

1. Вокруг определения сингулярной системы ................... 46

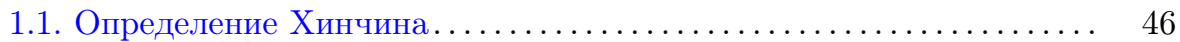

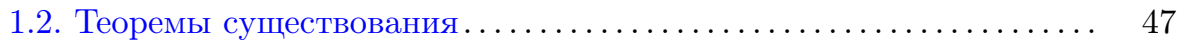

1.3. Сингулярные системы и наилучшие приближения............ 49

2. Подпространства, порожденные наилучшими приближениями . . . . . . 53

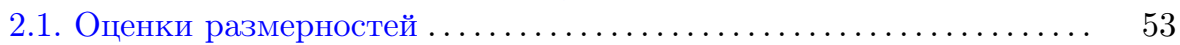

2.2. Вырождение размерности, $R(\Theta)=2 \ldots \ldots \ldots \ldots \ldots \ldots \ldots \ldots . \ldots \ldots$

2.3. Вырождение размерности, $R(\Theta)=3 \ldots \ldots \ldots \ldots \ldots \ldots \ldots \ldots . \ldots \ldots$

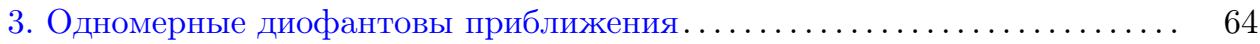

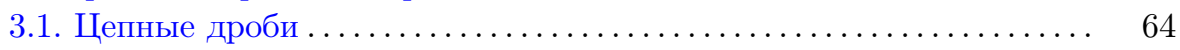

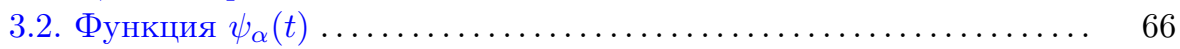

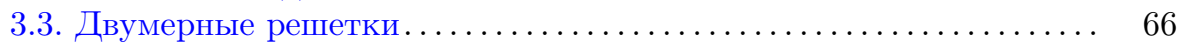

4. Сингулярные системы в задаче о совместных диофантовых приближе-

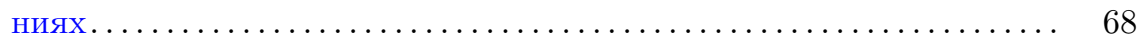

4.1. Независимость векторов наилучших приближений .......... 68

4.2. Вырождение размерности наилучших совместных приближений 71

Работа выполнена при поддержке РФФИ (грант № 09-01-00371а) и программы “Ведущие научные школы" (грант НШ-691.2008.1).

(C) Н. Г. Мощевитин, 2010 
5. Сингулярность и диофантов тип............................ 72

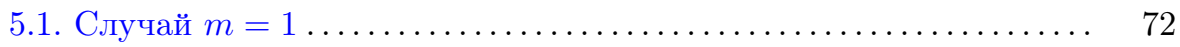

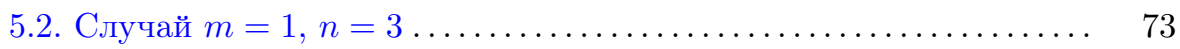

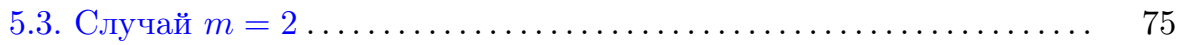

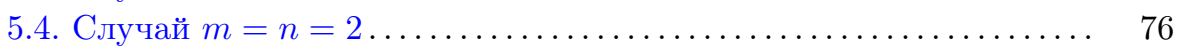

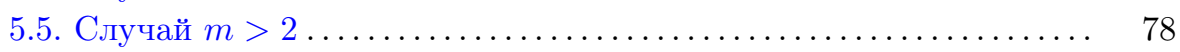

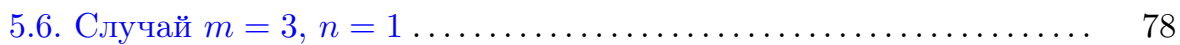

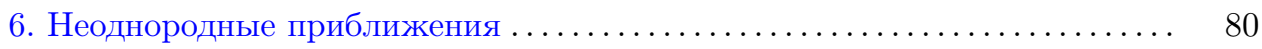

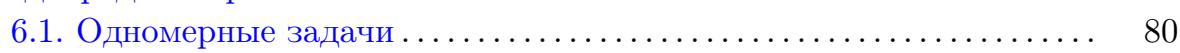

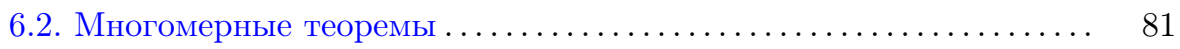

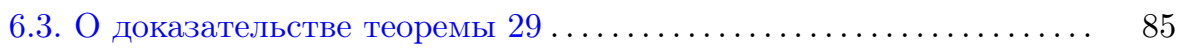

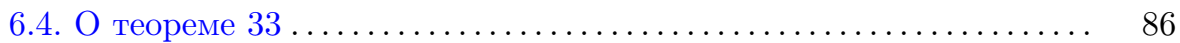

7. Пространства решеток ............................ 90

7.1. Метрическая теорема Давенпорта-Шмидта................ 90

7.2. Задача о последовательных минимумах .................. 91

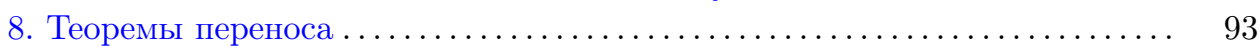

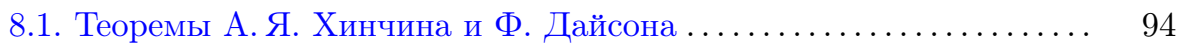

8.2. Результаты В. Ярника и А. Апфельбека...................... 94

8.3. Теоремы М. Лорана............................. 97

9. О размерности Хаусдорфа множеств сингулярных систем ........... 98

10. Приближения с целыми неотрицательными числами . . . . . . . . . . . 100

10.1. Двумерные приближения . . . . . . . . . . . . . . . . . . 100

10.2. О линейных формах с числом переменных $k>2 \ldots \ldots \ldots \ldots \ldots 101$

11. Задача В. В. Козлова................................. 102

11.1. Лемма Переса и теорема Халаса....................... 104

11.2. Индивидуальная возвращаемость ..................... 105

11.3. Равномерная возвращаемость . . . . . . . . . . . . . . . . . . . 108

12. Сингулярные системы специального вида.................... 111

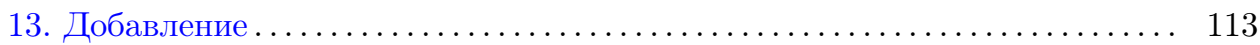

13.1. О лакунарных последовательностях ................... 113

13.2. О некоторых метрических результатах ................. 114

13.3. О применении метода Переса-Шлага ..................... 115

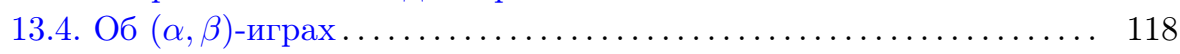

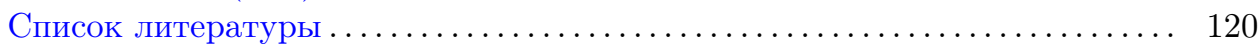

В 1926 г. А.Я. Хинчин обнаружил (см. [1]), что в двумерных задачах теории диофантовых приближений возникают явления, существенно отличающиеся от тех, которые имеют место в задаче о приближении рациональными числами одного иррационального числа. В частности, он конструктивно доказал существование двумерных вещественных векторов, "аномально хорошо" приближаемых рациональными числами (в смысле линейной формы и в смысле совместных приближений). В дальнейшем конструкция работы [1] была обобщена на случай произвольного количества линейных форм от произвольного количества целочисленных переменных, а матрицы коэффициентов линейных форм, допускающих аномально хорошие приближения, получили название сингулярных. 
В настоящей работе мы делаем обзор результатов из теории диофантовых приближений и ее приложений, тем или иным образом связанных с использованием сингулярных матриц А.Я. Хинчина. В основном, эта часть теории диофантовых приближений была построена в статьях А.Я. Хинчина [2]-[8] и В. Ярника [9]-[15].

Основополагающая работа [1] является одной из лучших (скорее, самой лучшей) работой А.Я. Хинчина в области теории диофантовых приближений. Многие статьи А. Я. Хинчина были недавно переизданы в книге его избранных трудов [16]. К сожалению, замечательные работы В. Ярника малодоступны, на долгое время они были забыты.

Данный обзор начинается с обсуждения определения сингулярной матрицы, теорем существования и связи с наилучшими диофантовыми приближениями (раздел 1). В разделе 2 излагаются общие результаты о подпространствах, порожденных наилучшими приближениями. Отдельно рассматривается задача о совместных приближениях (раздел 4) и восходящая к В. Ярнику задача о связи равномерных и индивидуальных диофантовых характеристик (раздел 5). Там же дается усиление одного результата В. Ярника. В нескольких местах излагаются результаты автора о вырождении размерности подпространств, порожденных наилучшими приближениями. Задачам о неоднородных линейных диофантовых приближениях посвящен раздел 6. В разделе 7 рассматриваются задачи теории решеток, связанные с вопросами диофантовых приближений. В разделе 8 перечисляются классические и современные результаты, касающиеся принципа переноса. В разделе 9 собраны известные автору утверждения о размерности Хаусдорфа множеств сингулярных матриц.

Вопросы, связанные с применением теории диофантовых приближений, естественным образом возникают в некоторых задачах классической механики, имеющих отношение к так называемой проблеме “малых знаменателей”, например, в теории возмущений условно периодических движений (см. [17]). В частности, оказалось, что сингулярные системы А.Я. Хинчина могут быть применены для построения систем с "быстро разбегающимися траекториями", что было сделано В. В. Козловым и Н. Г. Мощевитиным [18]. Более того, многомерный диофантов анализ оказался основным инструментом при решении задачи В. В. Козлова об осцилляции интеграла условно периодической функции. Этой задаче посвящен раздел 11 настоящего обзора.

В добавлении (раздел 13) мы хотели упомянуть ряд утверждений и теорем, имеющих отношение к некоторым затронутым вопросам и их развитию. Это, во-первых, результаты, которые получаются с помощью оригинального метода, предложенного недавно Ю. Пересом и В. Шлагом [19]. Во-вторых, это основные понятия и некоторые результаты теории выигрышных множеств, построенной В. М. Шмидтом [20].

Некоторые вопросы, затронутые в настоящей статье (равно как и многие другие результаты теории диофантовых приближений), нашли свое отражение в замечательном обзоре М. Вальдшмидта [21]. Вопросам теории диофантовых приближений посвящены ставшие классическими монографии Дж.Ф. Коксмы [22], Дж. В. С. Касселса [23] и В. М. Шмидта [24].

Кроме перечисленных выше, в настоящем обзоре затронуты и некоторые другие задачи. Это, например, задача о диофантовых приближениях с поло- 
жительными числами (раздел 10) и проблемы существования матриц с чрезвычайно сингулярными диофантовыми свойствами (раздел 12).

Автор считает своей приятной обязанностью поблагодарить всех участников теоретико-числовых семинаров и спецкурсов механико-математического факультета МГУ и Независимого московского университета за многочисленные обсуждения задач и теорем из настоящего обзора. В особенности автор благодарен И. П. Рочеву и О.Н. Герману. Также автор глубоко признателен В. А. Быковскому за исправление ряда неточностей, имевшихся в первоначальном варианте статьи.

\section{1. Вокруг определения сингулярной системы}

Всюду ниже $\mathbf{x}=\left(x_{1}, \ldots, x_{m}\right)$ обозначает целочисленный вектор. Через $\|\cdot\|$ обозначено расстояние до ближайшего целого числа. Рассматривается матрица

$$
\Theta=\left(\begin{array}{ccc}
\theta_{1}^{1} & \ldots & \theta_{1}^{m} \\
\ldots \ldots & \ldots & \ldots \\
\theta_{n}^{1} & \ldots & \theta_{n}^{m}
\end{array}\right)
$$

состоящая из вещественных чисел $\theta_{j}^{i}, 1 \leqslant j \leqslant n, 1 \leqslant i \leqslant m$, и соответствующая ей система линейных форм

$$
\mathbf{L}(\mathbf{x})=\mathbf{L}_{\Theta}(\mathbf{x})=\left\{L_{j}(\mathbf{x}), 1 \leqslant j \leqslant n\right\}, \quad L_{j}(\mathbf{x})=\sum_{i=1}^{m} \theta_{j}^{i} x_{i} .
$$

Через ${ }^{t} \Theta$ будем обозначать матрицу, транспонированную к матрице $\Theta$.

Из теоремы Минковского о выпуклом теле следует, что для любой матрицы $\Theta$ и при любом вещественном $t \geqslant 1$ система диофантовых неравенств

$$
\max _{1 \leqslant j \leqslant n}\left\|L_{j}(\mathbf{x})\right\| \leqslant \frac{1}{t}, \quad 0<\max _{1 \leqslant i \leqslant m}\left|x_{i}\right| \leqslant t^{n / m},
$$

имеет целочисленное решение $\mathbf{x} \in \mathbb{Z}^{m}$.

1.1. Определение Хинчина. Сначала мы сформулируем определение сингулярной системы именно в том виде, в каком его давал А.Я. Хинчин (см. [7]). Матрица $\Theta$ (или, как писал сам А.Я. Хинчин, набор вещественных чисел $\theta_{j}^{i}$ с $1 \leqslant i \leqslant m$ и $\left.1 \leqslant j \leqslant n\right)$ представляет собой сингулярную систему, если при любом вещественном $\varepsilon>0$ найдется некоторое $t_{0}=t_{0}(\varepsilon)$ такое, что при всяком $t \geqslant t_{0}$ система диофантовых неравенств

$$
\max _{1 \leqslant j \leqslant n}\left\|L_{j}(\mathbf{x})\right\| \leqslant \frac{1}{t}, \quad 0<\max _{1 \leqslant i \leqslant m}\left|x_{i}\right|<\varepsilon t^{n / m},
$$

имеет целочисленное решение $\mathbf{x} \in \mathbb{Z}^{m}$.

Отметим, что матрицу $\Theta$, не являющуюся сингулярной, А. Я. Хинчин называл регулярной (он использовал терминологию регулярная система чисел $\theta_{j}^{i}$ ).

Таким образом, матрица $\Theta$ является регулярной, если найдется положительное $\mu(\Theta)$ такое, что для некоторой последовательности положительных 
чисел $t_{\nu}$, стремящейся к бесконечности, в областях пространства $\mathbb{R}^{n}$, задаваемых неравенствами

$$
\max _{1 \leqslant j \leqslant n}\left\|L_{j}(\mathbf{x})\right\| \leqslant \frac{1}{t_{\nu}}, \quad 0<\max _{1 \leqslant i \leqslant m}\left|x_{i}\right| \leqslant \mu(\Theta) t_{\nu}^{n / m},
$$

отсутствуют ненулевые целые точки. То же самое можно сказать несколько по-другому (как, например, это сделано в работе Г. Давенпорта и В. М. Шмидта [25]): матрица $\Theta$ является регулярной, если найдется положительное $\mu(\Theta)$ такое, что для некоторой последовательности положительных чисел $t_{\nu}$, стремящейся к бесконечности, в областях пространства $\mathbb{R}^{n}$, задаваемых неравенствами

$$
\max _{1 \leqslant j \leqslant n}\left\|L_{j}(\mathbf{x})\right\| \leqslant \frac{\mu(\Theta)}{t_{\nu}}, \quad 0<\max _{1 \leqslant i \leqslant m}\left|x_{i}\right| \leqslant \mu(\Theta) t_{\nu}^{n / m},
$$

отсутствуют ненулевые целые точки.

В этой статье мы будем использовать, в основном, матричную терминологию. Тем не менее, иногда удобно употреблять понятие сингулярной системы вещественных чисел.

1.2. Теоремы существования. При $n=m=1$, как легко видеть, сингулярными системами в смысле определения Хинчина являются только рациональные числа $\theta_{1}^{1}$ (это утверждение мы прокомментируем ниже в п. 3.1). Впервые существование сингулярных систем было доказано А. Я. Хинчиным в 1926 г. в работе [1] при $m=2, n=1$ и при $m=1, n=2$. Здесь мы приведем формулировки соответствующих утверждений ([1; Hilfssatz I и Satz 2], см. также [23; гл. V, теорема XIV]).

ТЕОРема 1. Пусть $\psi(t)$ - положительная, непрерывная и убывающая к нулю при $t \rightarrow+\infty$ функиия вещественного переменного $t$. Тогда существуют два линейно независимых вместе с единицей над $\mathbb{Z}$ вещественных числа $\theta^{1}$ и $\theta^{2}$ такие, что для каждого достаточно большого $t$ система диофантовых неравенств

$$
\left\|x_{1} \theta^{1}+x_{2} \theta^{2}\right\|<\psi(t), \quad 0<\max _{j=1,2}\left|x_{j}\right|<t,
$$

имеет иелочисленное решение $\left(x_{1}, x_{2}\right) \in \mathbb{Z}^{2}$.

Теорема 2. Пусть $\psi(t)$ - положительная, непрерьвная и убывающая $к$ нулю при $t \rightarrow+\infty$ функиия вещественного переменного $t$ и при этом функиця $t \psi(t)$ монотонно возрастает $\kappa$ бесконечности при $t \rightarrow+\infty$. Тогда существуют два линейно независимых вместе с единицей над $\mathbb{Z}$ вещественных числа $\theta_{1}, \theta_{2}$ такие, что для каждого достаточно большого $t$ система диобантовых неравенств

$$
\max _{j=1,2}\left\|x \theta_{j}\right\|<\psi(t), \quad 0<x<t
$$

разрешима в челых числах $x$.

Итак, в случае $m=2$ и $n=1$ теорема 1 устанавливает существование сингулярных систем с соответствующей функцией $\psi(t)$, сколь угодно быстро стремящейся к нулю. В случае $m=1$ и $n=2$ теорема 2 устанавливает существование сингулярных систем только в случае, когда соответствующая функция $t \mapsto t \psi(t)$ монотонно возрастает к бесконечности. 
В связи с этим уместно следующее определение. Пусть непрерывная функция $\psi(t)$ монотонно убывает к нулю и $\psi(t)=o\left(t^{-m / n}\right)$ при $t \rightarrow+\infty$. Матрицу $\Theta$ (или набор $m n$ вещественных чисел) мы будем называть $\psi$-сингулярным, если при всяком достаточно большом $t$ система диофантовых неравенств

$$
\max _{1 \leqslant j \leqslant n}\left\|L_{j}(\mathbf{x})\right\| \leqslant \psi(t), \quad 0<\max _{1 \leqslant i \leqslant m}\left|x_{i}\right|<t,
$$

имеет целочисленное решение $\mathbf{x} \in \mathbb{Z}^{m}$.

Сформулированная выше теорема 1, в более сильном варианте, непосредственно обобщается на случай произвольной размерности следующим образом.

Теорема 3. Пусть $n$ - произвольное натуральное число, $m$ - натуральное число, не меньшее, чем 2. Предположим также, что $\psi(t)$ - положительная, непрерьвная и убъвающая $\kappa$ нулю при $t \rightarrow+\infty$ функция вещественного переменного $t$. Рассмотрим множество $\mathscr{M} \subset \mathbb{R}^{m n}$, состоящее из матрич $\Theta$ таких, что над $\mathbb{Z}$

- числа $\theta_{j}^{i}$ c $1 \leqslant i \leqslant m, 1 \leqslant j \leqslant n$ линейно независимы вместе с единицей

- матрица $\Theta$ является $\psi$-сингулярной.

Тогда для любого отрытого множества $\mathscr{G} \subset \mathbb{R}^{m n}$ пересечение $\mathscr{M} \cap \mathscr{G}$ имеет мощность континуума.

Отметим, что при $m=1$ дело обстоит несколько по-другому. Итак, пусть $m=1$ и $\Theta=\left\{\theta_{1}, \ldots, \theta_{n}\right\}$ есть некоторый набор вещественных чисел. Условимся обозначать через $\operatorname{dim}_{\mathbb{Z}} \Theta$ максимально возможное количество линейно независимых над $\mathbb{Z}$ чисел из набора $\theta_{1}, \ldots, \theta_{n}, 1$. Сформулируем обобщение теоремы 2 .

TEOPEMA 4. Пусть $m=1 u n \geqslant 2$.

(i) Пусть $\psi(t)$ - положительная, непрерывная и убывающая $к$ нулю при $t \rightarrow+\infty$ функция вещественного переменного $t$, причем

$$
\lim _{t \rightarrow \infty} \psi(t) t=+\infty
$$

Рассмотрим множество $\mathscr{M} \subset \mathbb{R}^{n}$ наборов вещественных чисел $\Theta=\left\{\theta_{j}\right.$, $1 \leqslant j \leqslant n\}$ maкux, чmо

$-\operatorname{dim}_{\mathbb{Z}} \Theta=n+1$,

- система $\Theta$ является $\psi$-сингулярной.

Тогда для любого отрытого множества $\mathscr{G} \subset \mathbb{R}^{n}$ пересечение $\mathscr{M} \cap \mathscr{G}$ имеет мошность континуума.

(ii) Пусть $n \geqslant 2$ и для положительнозначной функции $\psi(t)$ выполнено

$$
\limsup _{t \rightarrow+\infty} \psi(t) t<+\infty
$$

Тогда, если набор $\Theta=\left\{\theta_{1}, \ldots, \theta_{n}\right\}$ представляет собой $\psi$-сингулярную систему, то $\operatorname{dim}_{\mathbb{Z}} \Theta \leqslant 2$. Более того, если

$$
\limsup _{t \rightarrow+\infty} \psi(t) t=0
$$

mо $\operatorname{dim}_{\mathbb{Z}} \Theta=1$, m.е. все числа $\theta_{j} c 1 \leqslant j \leqslant n$ суть рациональные. 
Теорема 2 и часть (i) теоремы 4 имеются у В. Ярника [14], частный случай рассмотрен у К. Шаботи и Е. Лютц [26]. Отметим, что В. Ярник формулирует и доказывает несколько более сильный результат: существование матриц $\Theta$, состоящих из вещественных чисел $\theta_{j}^{i}$ в теореме 3 , алгебраически независимых в совокупности.

Утверждение (ii) теоремы 4 очевидным образом вытекает из следствия 5 п. 4.1 (с. 70), которое тоже принадлежит В. Ярнику (см. [9] и [14]), в предположении (6) результат мгновенно получается из следствия 1 п. 4.1. Таким образом, в случае $m=1$ и $n \geqslant 2$ при условии (5) нетривиальных сингулярных систем не существует.

Теорема 4 допускает некоторое уточнение, имеющееся у Ж. Леска [27].

Теорема 5 (ЖК. Леска [27]). Пусть $m=1 u n \geqslant 2$. Пусть $\psi(t)-$ положительная, непрерьвная и убывающая $к$ нулю при $t \rightarrow+\infty$ функиия вещественного переменного $t$, для которой

$$
\limsup _{t \rightarrow \infty} \psi(t) t=+\infty .
$$

Тогда множество $\psi$-сингулярных наборов $\Theta=\left\{\theta_{j}, 1 \leqslant j \leqslant n\right\}$, состоящее из алгебраически независимых вещественных чисел, в пересечении с произвольным отрытым множеством $\mathscr{G} \subset \mathbb{R}^{n}$ имеет мощность континуума.

Теорему 5 уместно сопоставить с формулой В. Ярника (53) и с теоремой 17. Отметим, что Ж. Леска в [28] получил $p$-адический вариант теоремы 3.

Приведем теорему существования, доказанную А. Апфельбеком [29].

Теорема 6 (А. Апфельбек [29]). Пусть $n, m \geqslant 2$. Пусть непрерывная функция $\psi(t)$ такова, что функиия $t \mapsto t \psi(t)$ убъвает. Тогда найдется $\psi$-сингулярная матрица $\Theta$, состоящая из линейно независимых вместе с единицей над $\mathbb{Z}$ чисел $\theta_{j}^{i}$, такая, что матрица ${ }^{t} \Theta$ тоже $\psi$-сингулярна.

Использование в определении сингулярной системы прилагательного "сингулярный" объясняется тем, что такие системы при каждом значении размерностей $m, n$, как отмечал сам А.Я. Хинчин (см. [7]), образуют множество нулевой меры Лебега. Этот факт связан с простым применением леммы Бореля-Кантелли. Подробное доказательство можно найти в [23; гл. V, §7]. Более сильный результат был получен Г. Давенпортом и В. М. Шмидтом [25]. Мы обсудим его ниже в п. 7.1.

1.3. Сингулярные системы и наилучшие приближения. Для целочисленного вектора $\mathbf{x}=\left(x_{1}, \ldots, x_{m}\right) \in \mathbb{Z}^{m}$ рассмотрим величины

$$
M(\mathbf{x})=\max _{1 \leqslant i \leqslant m}\left|x_{i}\right|, \quad \zeta(\mathbf{x})=\max _{1 \leqslant j \leqslant n}\left\|L_{j}(\mathbf{x})\right\| .
$$

Мы будем называть целочисленную точку $\mathbf{x}=\left(x_{1}, \ldots, x_{m}\right)$ наилучшим nриближением для матрицы $\Theta$, если

$$
\zeta(\mathbf{x})=\min _{\mathbf{x}^{\prime}} \zeta\left(\mathbf{x}^{\prime}\right),
$$

где минимум берется по всем ненулевым целым точкам $\mathbf{x}^{\prime}=\left(x_{1}^{\prime}, \ldots, x_{m}^{\prime}\right) \in \mathbb{Z}^{m}$, подчиненным условию

$$
0<M\left(\mathbf{x}^{\prime}\right) \leqslant M(\mathbf{x})
$$


При таком определении оказывается, что наилучшие приближения будут встречаться парами: вместе с каждой точкой $\mathbf{x}$, являющейся наилучшим приближением, наилучшим приближением будет также точка $-\mathbf{x}$. Однако, если рассмотреть такую пару наилучших приближений $\pm \mathbf{x}$, то для нее величины $M(\mathbf{x})$ и $\zeta(\mathbf{x})$ будут определяться однозначно и вне зависимости от выбора знака \pm .

Отметим следующее. Вообще говоря, может оказаться, что для двух целочисленных точек $\mathbf{x}_{1} \neq \pm \mathbf{x}_{2}$ с одинаковым значением $M\left(\mathbf{x}_{1}\right)=M\left(\mathbf{x}_{2}\right) \neq 0$ выполняется

$$
\max _{1 \leqslant j \leqslant n}\left\|L_{j}\left(\mathbf{x}_{1}\right)\right\|=\max _{1 \leqslant j \leqslant n}\left\|L_{j}\left(\mathbf{x}_{2}\right)\right\| .
$$

Но если, например, предположить, что все числа $\theta_{j}^{i}, 1 \leqslant i \leqslant m, 1 \leqslant j \leqslant n$, линейно независимы вместе с единицей над $\mathbb{Z}$, то равенство (9) невозможно. Таким образом, в случае, когда все числа $\theta_{j}^{i}$ с $1 \leqslant i \leqslant m, 1 \leqslant j \leqslant n$ линейно независимы вместе с единицей над $\mathbb{Z}$, наилучшие приближения можно перенумеровать и расположить в виде бесконечной последовательности

$$
\pm \mathbf{x}_{1}, \pm \mathbf{x}_{2}, \ldots, \pm \mathbf{x}_{\nu}, \pm \mathbf{x}_{\nu+1}, \ldots
$$

так, чтобы выполнялись неравенства

$$
\begin{gathered}
M\left(\mathbf{x}_{1}\right)<M\left(\mathbf{x}_{2}\right)<\cdots<M\left(\mathbf{x}_{\nu}\right)<M\left(\mathbf{x}_{\nu+1}\right)<\cdots, \\
\zeta\left(\mathbf{x}_{1}\right)>\zeta\left(\mathbf{x}_{2}\right)>\cdots>\zeta\left(\mathbf{x}_{\nu}\right)>\zeta\left(\mathbf{x}_{\nu+1}\right)>\cdots
\end{gathered}
$$

Для краткости мы будем использовать обозначения

$$
M_{\nu}=M\left(\mathbf{x}_{\nu}\right), \quad \zeta_{\nu}=\zeta\left(\mathbf{x}_{\nu}\right)
$$

В дальнейшем нам иногда придется иметь дело с наилучшими приближениями для матриц $\Theta$, не удовлетворяющих условию линейной независимости. В этом случае мы не можем определить последовательность точек $\mathbf{x}_{\nu}$ однозначно. Тем не менее, последовательности $(10),(11)$ величин $M\left(\mathbf{x}_{\nu}\right), \zeta\left(\mathbf{x}_{\nu}\right)$ однозначно определяются (только каждому из значений $M\left(\mathbf{x}_{\nu}\right), \zeta\left(\mathbf{x}_{\nu}\right)$, вообще говоря, может соответствовать несколько пар целочисленных точек $\pm \mathbf{x}_{\nu}$ и последовательности, вообще говоря, могут быть конечными).

В связи со сказанным выше мы даем следующее определение. Матрицу $\Theta$ мы будем называть правильной, если последовательности (10), (11) бесконечны и для каждого достаточно большого значения $\nu$ векторы $\mathbf{x}_{\nu}$ определены однозначно с точностью до знака.

Надо сделать еще одно замечание. Определим единичные векторы $\mathbf{e}^{k}, 1 \leqslant$ $k \leqslant n$ (у $k$-го вектора единица стоит на $k$-м месте, а остальные компоненты равны нулю), и рассмотрим в $\mathbb{R}^{n}$ набор, состоящий из $m+n$ векторов

$$
\theta^{1}, \ldots, \theta^{m}, \mathbf{e}^{1}, \ldots, \mathbf{e}^{n}
$$

(здесь $\theta^{j}$ обозначает $j$-й столбец матрицы $\Theta$ ). Легко видеть, что последовательности наилучших приближений $(10),(11)$ являются бесконечными в том и только том случае, когда набор векторов (12) является линейно независимым над $\mathbb{Z}$. В этом случае В. Ярник предлагал говорить, что матрица $\Theta$ является 
невырожденной (в настоящей статье мы тоже будем использовать эту терминологию). В противном случае последовательности (10), (11) будут конечными и для последнего значения индекса $\nu$ будет выполнено $\zeta_{\nu}=0$.

Если хотя бы одно из чисел $m$ или $n$ равно единице, то матрица $\Theta$ представляет собой один столбец или одну строку и может быть отождествлена просто с набором из $m$ или $n$ вещественных чисел, и можно говорить о величине $\operatorname{dim}_{\mathbb{Z}} \Theta$ как о характеристике этого набора вещественных чисел. Именно в этом смысле обозначение $\operatorname{dim}_{\mathbb{Z}} \Theta$ употреблялось в теореме 4. В этом же смысле это обозначение нам понадобится и в дальнейшем. В частности, получается, что для матрицы $\Theta$ величина $\operatorname{dim}_{\mathbb{Z}} \Theta$ определена только при $m=1$ или $n=1$. Например, при $m=1$ невырожденность матрицы $\Theta$ означает, что хотя бы одно из чисел $\theta_{j}=\theta_{j}^{1}$ является иррациональным, т. е. $\operatorname{dim}_{\mathbb{Z}} \Theta \geqslant 2$. При $n=1$ невырожденность матрицы $\Theta$ означает, что числа $1, \theta_{1}^{1}, \ldots, \theta_{1}^{m}$ линейно независимы над $\mathbb{Z}$, т. е. $\operatorname{dim}_{\mathbb{Z}} \Theta=m+1$.

Нам понадобится еще одна характеристика независимости. Для матрицы $\Theta$ через $\mathrm{DIM}_{\mathbb{Z}} \Theta$ обозначим максимальное количество векторов из набора (12) таких, что они линейно независимы над $\mathbb{Z}$. Заметим, что при $n=1$ всегда выполнено

$$
\mathrm{DIM}_{\mathbb{Z}} \Theta=\operatorname{dim}_{\mathbb{Z}} \Theta
$$

а при $m=1$ величина $\operatorname{DIM}_{\mathbb{Z}} \Theta$ может принимать только два значения $n$ и $n+1$. В последнем случае вместо равенства (13), естественно, имеет место равенство

$$
\operatorname{DIM}_{\mathbb{Z}}{ }^{t} \Theta=\operatorname{dim}_{\mathbb{Z}}{ }^{t} \Theta
$$

Очевидно, что для произвольной матрицы $\Theta$ имеют место неравенства

$$
n \leqslant \mathrm{DIM}_{\mathbb{Z}} \Theta \leqslant n+m .
$$

Невырожденность матрицы $\Theta$ эквивалентна равенству

$$
\mathrm{DIM}_{\mathbb{Z}} \Theta=m+n
$$

Поясним взаимосвязь между правильностью и невырожденностью матрицы $\Theta$. Если $m=1$ или $n=1$, то из невырожденности следует правильность. В других случаях это неверно. Приведем пример.

Пусть $\xi$ - иррациональное число, $m=n=2$ и матрица $\Theta$ имеет вид

$$
\Theta=\left(\begin{array}{ll}
\theta_{1}^{1} & \theta_{1}^{2} \\
\theta_{2}^{1} & \theta_{2}^{2}
\end{array}\right)=\left(\begin{array}{ll}
\xi & 0 \\
0 & \xi
\end{array}\right) .
$$

Тогда

$$
\operatorname{DIM}_{\mathbb{Z}} \Theta=\operatorname{DIM}_{\mathbb{Z}}{ }^{t} \Theta=4
$$

и матрица является невырожденной. Но, как легко видеть, каждому значению $\nu$ соответствуют векторы

$$
\left( \pm q_{\nu}, \pm q_{\nu}\right), \quad\left( \pm q_{\nu}, 0\right), \quad\left(0, \pm q_{\nu}\right)
$$

$\left(q_{\nu}-\right.$ знаменатель некоторой подходящей к $\xi$ дроби) с одинаковыми значениями величин $M_{\nu}, \zeta_{\nu}$. Однако набор вещественных чисел $1, \theta_{j}^{i}$ с $1 \leqslant i, j \leqslant 2$ линейно зависим над $\mathbb{Z}$, поскольку среди чисел $\theta_{j}^{i}$ имеются нули. 
Продолжим анализ понятия наилучшего приближения. Обозначим через $\mathbf{y}_{\nu}=\left(y_{1, \nu}, \ldots, y_{n, \nu}\right) \in \mathbb{Z}^{n}$ целочисленный вектор, состоящий из чисел $y_{j, \nu}$ таких, что

$$
\left\|L_{j}\left(\mathbf{x}_{\nu}\right)\right\|=\left|L_{j}\left(\mathbf{x}_{\nu}\right)+y_{j, \nu}\right|
$$

Нам понадобится обозначение

$$
\mathbf{z}_{\nu}=\left(x_{1, \nu}, \ldots, x_{m, \nu}, y_{1, \nu}, \ldots, y_{n, \nu}\right) \in \mathbb{Z}^{d}, \quad d=m+n,
$$

для “расширенного" вектора наилучших приближений.

То, что "расширенный” вектор

$$
\mathbf{z}=\left(x_{1}, \ldots, x_{m}, y_{1}, \ldots, y_{n}\right)
$$

является наилучшим приближением, означает, что внутри параллелепипеда

$$
\begin{array}{r}
\left\{\mathbf{z}^{\prime}=\left(x_{1}^{\prime}, \ldots, x_{m}^{\prime}, y_{1}^{\prime}, \ldots, y_{n}^{\prime}\right): M\left(\mathbf{x}^{\prime}\right) \leqslant M(\mathbf{x}),\right. \\
\left.\max _{1 \leqslant j \leqslant n}\left|L_{j}\left(\mathbf{x}^{\prime}\right)+y_{j}^{\prime}\right| \leqslant \max _{1 \leqslant j \leqslant n}\left|L_{j}(\mathbf{x})+y_{j}\right|\right\}
\end{array}
$$

нет других целых точек кроме 0. Более того, внутри параллелепипедов вида

$$
\begin{aligned}
\left\{\mathbf{z}^{\prime}=\left(x_{1}^{\prime}, \ldots, x_{m}^{\prime}, y_{1}^{\prime}, \ldots, y_{n}^{\prime}\right): M\left(\mathbf{x}^{\prime}\right) \leqslant M\left(\mathbf{x}_{\nu+1}\right),\right. \\
\left.\max _{1 \leqslant j \leqslant n}\left|L_{j}(\mathbf{x})+y_{j}^{\prime}\right| \leqslant \zeta_{\nu}=\max _{1 \leqslant j \leqslant n}\left|L_{j}\left(\mathbf{x}_{\nu}\right)+y_{j, \nu}\right|\right\}
\end{aligned}
$$

нет других целых точек кроме 0. Отсюда по теореме Минковского о выпуклом теле получаем, что

$$
\zeta_{\nu}^{n} M_{\nu+1}^{m} \leqslant 1
$$

Кроме того, отметим два простых и важных свойства.

1. Каждый расширенный вектор $\mathbf{z}_{\nu}$ является примитивным, т. е.

$$
\text { н.о.д. }\left(x_{1, \nu}, \ldots, x_{m, \nu}, y_{1, \nu}, \ldots, y_{n, \nu}\right)=1 \text {. }
$$

2. Любые два последовательных вектора наилучших приближений $\mathbf{z}_{\nu}, \mathbf{z}_{\nu+1}$ дополнимы до базиса решетки $\mathbb{Z}^{d}$.

Свойство матрицы $\Theta$ быть сингулярной легко может быть переформулировано на языке наилучших приближений.

ПреДЛОЖЕНИЕ 1. Пусть для непреръвной и монотонной бункции $\psi(t)$ въполнено условие $\psi(t)=o\left(t^{-m / n}\right)$ при $t \rightarrow+\infty$. Невырожденная матрица $\Theta$ является $\psi$-сингулярной тогда и только тогда, когда для всех достаточно больиих значений $\nu$ выполняется

$$
\zeta_{\nu} \leqslant \psi\left(M_{\nu+1}\right)
$$

Связь свойства системы вещественных чисел $\Theta$ быть сингулярной с понятием наилучших приближений в работах А. Я. Хинчина явно не отмечалась. Эта 
связь неявно используется в работах В. Ярника и в книге Дж. В. С. Касселса [23]. В частности, у В. Ярника (см. [9], [11], [13], [14]) используется кусочно постоянная функция

$$
\psi_{\Theta}(t)=\min _{\mathbf{x} \in \mathbb{Z}^{m}: 0<M(\mathbf{x}) \leqslant t} \max _{1 \leqslant j \leqslant n}\left\|L_{j}(\mathbf{x})\right\|,
$$

а в книге [23] в ряде доказательств (см. [23; гл. V, §§ 6, 7]) используется функция

$$
\eta(\rho)=\min _{\mathbf{x} \in \mathbb{Z}^{m}: 0<\left|x_{1}\right|^{2}+\cdots+\left|x_{m}\right|^{2} \leqslant \rho^{2}} \max _{1 \leqslant j \leqslant n}\left\|L_{j}(\mathbf{x})\right\| .
$$

Точки разрыва этих функций фактически определяют наилучшие приближения (у В. Ярника в sup-норме, как это сделано в настоящей статье, а в книге [23] - в евклидовой норме).

Заметим, наконец, что матрица $\Theta$ невырождена тогда и только тогда, когда функция $\psi_{\Theta}(t)$ никогда не обращается в нуль при $t \geqslant 1$. Невырожденная матрица $\Theta$ является $\psi$-сингулярной, если и только если при всех достаточно больших значениях $t$ для функции Ярника (20) выполнено

$$
\psi_{\Theta}(t) \leqslant \psi(t) .
$$

\section{2. Подпространства, порожденные наилучшими приближениями}

В этом разделе мы обсудим свойства линейных подпространств в $\mathbb{R}^{d}$, в которых содержатся векторы наилучших приближений $\mathbf{z}_{\nu}$.

2.1. Оценки размерностей. Под размерностью $\operatorname{dim} \Lambda$ решетки $\Lambda$ мы будем подразумевать размерность минимального линейного подпространства $\operatorname{span} \Lambda \subset \mathbb{R}^{d}$, содержащего решетку $\Lambda$. Рассмотрим в пространстве $\mathbb{R}^{d}$ линейное подпространство $\pi$. Пересечение $\pi \cap \mathbb{Z}^{d}$ представляет собой некоторую решетку $\Lambda=\Lambda(\pi)$ (возможно, состоящую из одной точки 0). Подпространство $\pi \subseteq \mathbb{R}^{d}$ мы будем называть вполне ращиональным, если

$$
\operatorname{dim} \pi=\operatorname{dim} \Lambda(\pi) .
$$

Далее, для данного линейного подпространства $\pi$ определим $\mathfrak{H}(\pi)$ как максимальное вполне рациональное подпространство, содержащееся в $\pi$. Определим также $\mathfrak{R}(\pi)$ как минимальное вполне рациональное подпространство, содержащее подпространство $\pi$. При этом

$$
\mathfrak{H}(\pi) \subseteq \pi \subseteq \mathfrak{R}(\pi) .
$$

Определим теперь векторы

$$
\bar{\theta}_{i}=\left(\theta_{i}^{1}, \ldots, \theta_{i}^{m}, 0, \ldots, 0,1,0, \ldots, 1\right), \quad 1 \leqslant i \leqslant n
$$

(единица здесь стоит на $(m+i)$-м месте).

Рассмотрим в $\mathbb{R}^{d}$ с $d=m+n$ линейное подпространство $\mathscr{N}(\Theta)$, натянутое на векторы $\bar{\theta}_{1}, \ldots, \bar{\theta}_{m}$, и ортогональное дополнение $\mathscr{L}(\Theta)$ к нему. Очевидно, что

$$
\operatorname{dim} \mathscr{N}(\Theta)=n, \quad \operatorname{dim} \mathscr{L}(\Theta)=m
$$


Рассмотрим теперь подпространства

$$
\mathfrak{H}_{\Theta}=\mathfrak{H}(\mathscr{L}(\Theta)), \quad \mathfrak{R}_{\Theta}=\mathfrak{R}(\mathscr{L}(\Theta)) .
$$

Для них

$$
\operatorname{DIM}_{\mathbb{Z}} \Theta+\operatorname{dim} \mathfrak{H}_{\Theta}=d, \quad \operatorname{DIM}_{\mathbb{Z}}{ }^{t} \Theta=\operatorname{dim} \mathfrak{R}_{\Theta} .
$$

В частности, невырожденность матрицы $\Theta$, согласно первому из равенств $(21)$, равносильна условию $\mathfrak{H}_{\Theta}=\{\mathbf{0}\}$.

Следующее утверждение общеизвестно. Оно фактически доказано В. Ярником в [13]. Случай $m=1$ есть у Дж. Лагариаса [30]. Случай $m=2$ и $n=1$ имеется у Г. Давенпорта и В. М. Шмидта в [31] (см. также работы автора [32], [33]).

Для правильной матрицы $\Theta$ рассмотрим величину

$$
\begin{gathered}
R(\Theta)=\min \left\{r: \text { найдутся подрешетка } \Lambda \subseteq \mathbb{Z}^{n+m}, \operatorname{dim} \Lambda=r, \text { и } \nu_{0} \in \mathbb{N}\right. \\
\text { такие, что } \left.\mathbf{z}_{\nu} \in \Lambda \text { для любого } \nu \geqslant \nu_{0}\right\} .
\end{gathered}
$$

Пусть $\Lambda_{\Theta}$ именно та решетка, которая фигурирует в определении величины $R(\Theta)$, и $\pi_{\Theta}=\operatorname{span} \Lambda_{\Theta}$. Положим

$$
K(\Theta)=\operatorname{dim}\left(\pi_{\Theta} \cap \mathscr{L}(\Theta)\right) \geqslant 1 .
$$

Последнее неравенство справедливо по той причине, что векторы наилучших приближений, лежащие в подпространстве $\pi_{\Theta}$, становятся сколь угодно близкими к подпространству $\mathscr{L}(\Theta)$.

ТЕОРема 7. Для правилъной матрицъ $\Theta$ выполнено следующее:

(i) $2 \leqslant R(\Theta) \leqslant \operatorname{DIM}_{\mathbb{Z}}{ }^{t} \Theta$;

(ii) если $m=1$, mо $R(\Theta)=\operatorname{DIM}_{\mathbb{Z}}{ }^{t} \Theta=\operatorname{dim}_{\mathbb{Z}} \Theta$;

(iii) если $m>n$, то $R(\Theta) \geqslant 3$;

(iv) если $K(\Theta)=1$ и $R(\Theta)>2$, mо $m<n$.

Поясним доказательства утверждений теоремы 7.

1. Нижняя оценка утверждения (i) следует из линейной независимости векторов $\mathbf{z}_{\nu}, \mathbf{z}_{\nu+1}$.

Для того чтобы доказать верхнюю оценку из (i) и утверждение (ii), введем несколько обозначений, которые понадобятся не только сейчас, но и в дальнейшем.

Расстояние между множествами $\mathscr{A}, \mathscr{B} \subset \mathbb{R}^{d}$ будем обозначать $\operatorname{dist}(\mathscr{A}, \mathscr{B})$ (нам удобно считать, что в данном случае имеется в виду расстояние в sup-норме). Если у нас имеется некоторая решетка $\Lambda \subset \mathbb{R}^{d}$ такая, что $\Lambda \cap \operatorname{span} \Lambda=\Lambda$, и если $\operatorname{dim} \Lambda<d$, то расстояние от точек множества $\mathbb{Z}^{d} \backslash \Lambda$ до линейного подпространства $\operatorname{span} \Lambda$ отделено от нуля. Его мы будем обозначать

$$
\rho(\Lambda)=\operatorname{dist}\left(\mathbb{Z}^{d} \backslash \Lambda, \operatorname{span} \Lambda\right)>0 .
$$

Если $\pi \subset \mathbb{R}^{d}$ есть вполне рациональное линейное подпространство, то будем обозначать

$$
\rho(\pi)=\rho\left(\pi \cap \mathbb{Z}^{d}\right) .
$$


2. Итак, теперь мы можем прокомментировать доказательство верхней оценки из утверждения (i).

Поскольку $\rho\left(\Re_{\Theta}\right)>0$, но $\operatorname{dist}\left(\mathbf{z}_{\nu}, \mathfrak{R}_{\Theta}\right) \leqslant \operatorname{dist}\left(\mathbf{z}_{\nu}, \mathscr{L}(\theta)\right) \rightarrow 0, \nu \rightarrow+\infty$, то при всех достаточно больших значениях $\nu$ имеем $\mathbf{z}_{\nu} \in \mathfrak{R}_{\Theta} \cap \mathbb{Z}^{n+1}$, и все доказано.

3. Прокомментируем доказательство утверждения (ii). Заметим, что при условии $m=1$ пространство $\mathscr{L}(\Theta)$ одномерно, $d=m+1$.

Если $\operatorname{dim}_{\mathbb{Z}} \Theta=r$, то это означает, что вектор $\Theta$ лежит в некотором вполне рациональном линейном подпространстве $\pi \subset \mathbb{R}^{n+1}$ размерности $r$ (которое и есть $\left.\mathfrak{R}_{\Theta}\right)$ и не лежит ни в каком вполне рациональном подпространстве меньшей размерности.

Оценка $R(\Theta) \leqslant r$ следует из верхней оценки утверждения (i).

Если $R(\Theta)<r$, то, поскольку $\operatorname{dist}\left(\mathbf{z}_{\nu}, \mathscr{L}(\theta)\right) \rightarrow 0$ при $\nu \rightarrow+\infty$, получается, что $\Theta$ лежит в некотором вполне рациональном подпространстве размерности $<r$, что невозможно.

Итак, $R(\Theta)=r$, и утверждение (ii) доказано.

4. Для доказательства утверждения (iii) надо воспользоваться неравенством (18), из которого при $m>n$ получаем

$$
\zeta_{\nu} M_{\nu+1} \rightarrow 0, \quad \nu \rightarrow+\infty
$$

В случае $R(\Theta)=2$ находим, что в двумерном подпространстве $\pi_{\Theta}=\operatorname{span} \Lambda_{\Theta}$ (здесь $\Lambda_{\Theta}$ есть именно та решетка, которая фигурирует в определении величины $R(\Theta)$ ) находится одномерное подпространство $\pi_{\Theta} \cap \mathscr{L}(\Theta)$, не содержащее ненулевых точек решетки $\Lambda_{\Theta}$ (ибо $\left.\mathfrak{H}_{\Theta}=\{\boldsymbol{0}\}\right)$. Теперь (25) противоречит предложению 2 , которое будет доказано в п. 3.3 (норма $|\cdot|$. будет индуцирована sup-нормой в $\mathbb{R}^{d}$ ).

5. Для доказательства утверждения (iv) надо снова воспользоваться неравенством (18), из которого при $m \geqslant n$ будет следовать, что

$$
\zeta_{\nu} M_{\nu+1} \leqslant 1 \quad \forall \nu
$$

Далее, рассмотрим вполне рациональное подпространство $\pi_{\Theta}$ и одномерное подпространство $\ell_{\Theta}=\pi_{\Theta} \cap \mathscr{L}(\Theta)$. Подпространство $\ell_{\Theta}$ не лежит ни в каком собственном вполне рациональном подпространстве подпространства $\pi_{\Theta}$. Применяя предложение 3 из п. 4.1 (с. 71), получаем

$$
\zeta_{\nu} M_{\nu+1} \rightarrow+\infty, \quad \nu \rightarrow+\infty
$$

что есть противоречие.

Хочется заметить, что в пункте 4 выше мы фактически доказали несколько более общее утверждение: в дополнение к утверждению (iii) теоремы 7 мы формулируем следующий результат.

ТеОРема 8. Пусть $2 \leqslant m \leqslant n$ и правильная матрица $\Theta$ является $\psi$-сингулярной с некоторой функиией $\psi(t)=o\left(t^{-1}\right)$ nри $t \rightarrow+\infty$. Тогда $R(\Theta) \geqslant 3$.

В условиях теоремы 8 при $m=n \geqslant 2$ требуется, чтобы матрица $\Theta$ была сингулярна в смысле оригинального определения А.Я. Хинчина; при $n>m$ требуется нечто большее. 
ЗАмечАниЕ. Во всех случаях, когда $R(\Theta) \geqslant 3$, найдется бесконечно много значений индекса $\nu$ таких, что векторы наилучших приближений $\mathbf{z}_{\nu-1}, \mathbf{z}_{\nu}, \mathbf{z}_{\nu+1}$ линейно независимы.

Как мы увидим в следующем пункте, подпространство $\pi_{\Theta}$ может иметь малую размерность $R(\Theta)$. Тем не менее, такое вырождение размерности накладывает иногда довольно жесткие условия на элементы матрицы $\Theta$.

Теорема 9. Пусть матрица $\Theta$ правильная и $R(\Theta) \leqslant n+K(\Theta)-1$. Тогда матрица $\Theta$ состоит из чисел $\theta_{j}^{i}$, связанных алгебраическим соотношением степени $\leqslant \min (m, R(\Theta)-K(\Theta)+1)$.

Теорема 9 практически очевидна. Подпространство $\mathscr{L}(\Theta)$ порождено векторами

$$
\underline{\theta}^{1}=\left(\begin{array}{c}
-1 \\
0 \\
\vdots \\
0 \\
\theta_{1}^{1} \\
\vdots \\
\theta_{n}^{1}
\end{array}\right), \quad \underline{\theta}^{2}=\left(\begin{array}{c}
0 \\
-1 \\
\vdots \\
0 \\
\theta_{1}^{2} \\
\vdots \\
\theta_{n}^{2}
\end{array}\right), \quad \ldots, \quad \underline{\theta}^{m}=\left(\begin{array}{c}
0 \\
0 \\
\vdots \\
-1 \\
\theta_{1}^{m} \\
\vdots \\
\theta_{n}^{m}
\end{array}\right) .
$$

В подпространстве $\pi_{\Theta}$ имеется базис из $R(\Theta)$ независимых целочисленных векторов

$$
\mathbf{u}^{j}=\left(\begin{array}{c}
u_{1}^{j} \\
\vdots \\
u_{m+n}^{j}
\end{array}\right), \quad 1 \leqslant j \leqslant R(\Theta) .
$$

Поскольку $\operatorname{dim} \operatorname{span}\left(\pi_{\Theta} \cup \mathscr{L}(\Theta)\right)=R(\Theta)+m-K(\Theta)$ и выполнено $(22)$, то векторы $\underline{\theta}^{1}, \ldots, \underline{\theta}^{m}, \mathbf{u}^{1}, \ldots, \mathbf{u}^{R(\Theta)}$ линейно зависимы над $\mathbb{R}$. Более того, поднабор

$$
\underline{\theta}^{1}, \ldots, \underline{\theta}^{m}, \mathbf{u}^{1}, \ldots, \mathbf{u}^{r}, \quad r=R(\Theta)-K(\Theta)+1, \quad 1 \leqslant r \leqslant n,
$$

тоже состоит из линейно зависимых над $\mathbb{R}$ векторов. По условию теоремы $m+R(\Theta)-K(\Theta)+1 \leqslant m+n$. Таким образом, у матрицы размера $(m+n) \times$ $(m+R(\Theta)-K(\Theta)+1)$, составленной из координат векторов $(26)$, все миноры максимального порядка являются нулевыми.

Так как векторы $\mathbf{u}^{1}, \ldots, \mathbf{u}^{r}$ независимы, то найдется набор индексов

$$
1 \leqslant i_{1}<\cdots<i_{r} \leqslant d
$$

такой, что

$$
\operatorname{det}\left(\begin{array}{ccc}
u_{i_{1}}^{1} & \ldots & u_{i_{1}}^{r} \\
\ldots \ldots & \ldots & \ldots \\
u_{i_{r}}^{1} & \ldots & u_{i_{r}}^{r}
\end{array}\right) \neq 0 .
$$

Набор, состоящий из $r$ индексов $1 \leqslant i_{1}<\cdots<i_{r} \leqslant d$, дополним до набора, состоящего из $r+m$ индексов, вида

$$
1,2, \ldots, m, j_{1}, \ldots, j_{r} ; \quad m<j_{1}<\cdots<j_{r} \leqslant d .
$$


По условию

$$
\operatorname{det}\left(\begin{array}{cccccc}
-1 & \ldots & 0 & u_{1}^{1} & \ldots & u_{1}^{r} \\
\ldots \ldots \ldots & \ldots \ldots \ldots \ldots \ldots & \ldots & \ldots \\
0 & \ldots & -1 & u_{m}^{1} & \ldots & u_{m}^{r} \\
\theta_{j_{1}}^{1} & \ldots & \theta_{j_{1}}^{m} & u_{j_{1}}^{1} & \ldots & u_{j_{1}}^{r} \\
\ldots \ldots & \ldots & \ldots & \ldots & \ldots & \ldots \\
\theta_{j_{r}}^{1} & \ldots & \theta_{j_{r}}^{m} & u_{j_{r}}^{1} & \ldots & u_{j_{r}}^{r}
\end{array}\right)=0 .
$$

Из столбцов с $u^{j}$ этого определителя можно выбрать ненулевой минор, отличный от правого нижнего. Поэтому равенство нулю определителя задает на элементы матрицы $\Theta$ нетривиальное алгебраическое соотношение степени $\leqslant \min (m, r)$.

Из утверждения (i) теоремы 7 и второго из равенств (21) сразу делаем следующий вывод.

СлеДСтвиЕ 1. Пусть матрица $\Theta$ правилъна. Тогда, если $K(\Theta)=m$, mо $\pi_{\Theta}=\Re_{\Theta}$ u $R(\Theta)=\mathrm{DIM}_{\mathbb{Z}}{ }^{t} \Theta$.

Из теорем 7, 9 и неравенства (22) получаем

СледСтвиЕ 2. Пусть матрица $\Theta$ правилъна. Тогда:

(i) если элементы матрицы $\Theta$ алгебраически независимы в совокупности, mo $R(\Theta) \geqslant n+1$;

(ii) если $(m, n) \neq(1,1)$ и элементы матрицы $\Theta$ алгебраически независимы в совокупности, то $R(\Theta) \geqslant 3$.

Для случая $m=n$ из теоремы 7 (утверждение (iv)) и теоремы 9 вытекают следующие утверждения.

СлЕДСтвИЕ 3. (i) Если для правилъной матрицъ $\Theta$ известно, ито $m=$ $n>1$ и $R(\Theta) \leqslant n+1$, то элементы матрицы $\Theta$ алгебраически зависимы.

(ii) Если для правильной матрицы $\Theta$ известно, что $m=n>1$ и $K(\Theta)=1$, то $R(\Theta)=2$ и элементы матричы $\Theta$ алгебраически зависимы.

СлЕДСТвиЕ 4. Пусть $m=n=2$ и матрица $\Theta$ правильна. Тогда:

(i) если $K(\Theta)=1$, то $R(\Theta)=2$;

(ii) если $K(\Theta)=2$, mо $\pi_{\Theta} \supseteq \mathscr{L}(\Theta)$ u $R(\Theta)=\operatorname{DIM}_{\mathbb{Z}}{ }^{t} \Theta$.

В частности, если $\mathrm{DIM}_{\mathbb{Z}}{ }^{t} \Theta=4$, то либо $R(\Theta)=2$, либо $R(\Theta)=4$.

2.2. Вырождение размерности, $R(\Theta)=2$. Пусть $\xi \in(0,1)$ есть иррациональное число и $p_{\nu} / q_{\nu}, \nu=1,2,3, \ldots,-$ все подходящие дроби к числу $\xi$. Пусть $m \geqslant 2$ и $n \geqslant 3$. Предположим, что элементы $\theta_{j}^{i}$ с $1 \leqslant i \leqslant 2$ и $1 \leqslant j \leqslant n$ связаны соотношениями

$$
\theta_{j}^{2}=-\xi \theta_{j}^{1}, \quad 1 \leqslant j \leqslant n,
$$

и рассмотрим матрицу

$$
\Theta=\left(\begin{array}{ccccc}
\theta_{1}^{1} & \theta_{1}^{2} & \theta_{1}^{3} & \ldots & \theta_{1}^{m} \\
\ldots \ldots \ldots \ldots \ldots \ldots & \ldots \ldots & \ldots \\
\theta_{n}^{1} & \theta_{n}^{2} & \theta_{3}^{n} & \ldots & \theta_{n}^{m}
\end{array}\right)=\left(\begin{array}{ccccc}
\theta_{1}^{1} & -\xi \theta_{1}^{1} & \theta_{1}^{3} & \ldots & \theta_{1}^{m} \\
\ldots \ldots \ldots \ldots \ldots & \ldots \ldots \ldots \ldots \ldots \\
\theta_{n}^{1} & -\xi \theta_{n}^{1} & \theta_{n}^{3} & \ldots & \theta_{n}^{m}
\end{array}\right)
$$


(если $m=2$, то в этой матрице два столбца). Отметим, что все элементы матрицы (28) при надлежащем выборе величин $\theta_{j}^{1}, j=1,2,3, \ldots$, могут быть линейно независимы вместе с единицей над $\mathbb{Z}$. Тогда

$$
\operatorname{DIM}_{\mathbb{Z}} \Theta=\operatorname{DIM}_{\mathbb{Z}}{ }^{t} \Theta=m+n
$$

и матрица правильна. В то же время условия (27) показывают, что элементы матрицы (28) всегда представляют собой набор алгебраически зависимых чисел (поэтому теорема 10, которую мы формулируем ниже, согласуется с теоремой 9).

ТеОрема 10. Пусть $2 \leqslant m<n$. Тогда для почти всех наборов из $n(m-1)$ чисел $\left(\theta_{1}^{i}, \theta_{2}^{i}, \ldots, \theta_{n}^{i}\right), i=1,3,4, \ldots, m, \operatorname{maких,~что~} 1 / 3<\theta_{1}^{1}, \theta_{2}^{1}, \ldots, \theta_{n-1}^{1}<$ $2 / 3<\theta_{n}^{1}<1, \theta_{j}^{i} \in(0,1)$ c $3 \leqslant i \leqslant m u 1 \leqslant j \leqslant n$, последовательность векторов наилучших приближений для матрицы (28) отличается от последовательности векторов

$$
(p_{\nu}, q_{\nu}, \underbrace{0, \ldots, 0}_{m+n-2}), \quad \nu=1,2,3, \ldots,
$$

не более чем на конечное число точек и, следовательно, $R(\Theta)=2$.

СлЕДСТВИЕ. При $m \geqslant 2 u n>m$ существуют матрищы $\Theta$, состоящие из линейно независимых вместе с единицей над $\mathbb{Z}$ элементов, такие, что $R(\Theta)=2$.

ДокАЗАТЕЛЬСТво теОРемы 10. Заметим, что

$$
\max _{1 \leqslant j \leqslant n}\left\|p_{\nu} \theta_{j}^{1}+q_{\nu} \theta_{j}^{2}\right\|=\max _{1 \leqslant j \leqslant n}\left\|\left(p_{\nu}-q_{\nu} \xi\right) \theta_{j}^{1}\right\|=\left|\left(p_{\nu}-q_{\nu} \xi\right) \theta_{n}^{1}\right| .
$$

С другой стороны, из свойств цепных дробей (см. п. 3.1, формулы (42), (43)) получаем, что

$$
\max _{1 \leqslant j \leqslant n}\left\|p_{\nu} \theta_{j}^{1}+q_{\nu} \theta_{j}^{2}\right\|=\left|\left(p_{\nu}-q_{\nu} \xi\right) \theta_{n}^{1}\right|<\frac{1}{q_{\nu+1}} .
$$

Чтобы вектор $\left(p_{\nu}, q_{\nu}\right)$ был вектором наилучшего приближения для матрицы $(28)$, а вектор $\left(p_{\nu+1}, q_{\nu+1}\right)$ был в точности следующим вектором наилучшего приближения для матрицы (28), достаточно, чтобы выполнялось неравенство

$$
\min _{\left(x_{1}, \ldots, x_{m} ; y_{1}, \ldots, y_{n}\right)} \max _{1 \leqslant j \leqslant n}\left|\left(x_{1}-\xi x_{2}\right) \theta_{j}^{1}+x_{3} \theta_{j}^{3}+\cdots+x_{m} \theta_{j}^{m}+y_{j}\right|>\left|\left(p_{\nu}-q_{\nu} \xi\right) \theta_{n}^{1}\right|,
$$

где минимум берется по целым точкам $\left(x_{1}, \ldots, x_{m} ; y_{1}, \ldots, y_{n}\right)$ таким, что

$$
0 \neq \max _{1 \leqslant i \leqslant m}\left|x_{i}\right| \leqslant q_{\nu+1}, \quad \max _{1 \leqslant j \leqslant n}\left|y_{j}\right| \leqslant 1+\left|x_{1}-\xi x_{2}\right|+\left|x_{3}\right|+\cdots+\left|x_{m}\right|
$$

и

$$
\left(x_{1}, \ldots, x_{m} ; y_{1}, \ldots, y_{n}\right) \neq \pm(p_{\nu}, q_{\nu}, \underbrace{0, \ldots, 0}_{m+n-2}), \pm(p_{\nu+1}, q_{\nu+1}, \underbrace{0, \ldots, 0}_{m+n-2}) .
$$


Если $x_{3}=\cdots=x_{m}=y_{1}=\cdots=y_{n}=0$, то от свойств чисел $\theta_{j}^{i}$ ничего не зависит и

$$
\max _{1 \leqslant j \leqslant n}\left|\left(x_{1}-\xi x_{2}\right) \theta_{j}^{1}+x_{3} \theta_{j}^{3}+\cdots+x_{m} \theta_{j}^{m}+y_{j}\right|=\left|\left(x_{1}-\xi x_{2}\right) \theta_{n}^{1}\right|>\left|\left(p_{\nu}-q_{\nu} \xi\right) \theta_{n}^{1}\right|,
$$

поскольку $p_{\nu}<q_{\nu}$ и подходящие дроби задают наилучшие одномерные приближения (см. п. 3.1).

Условие (30) будет выполнено, если для каждого рассматриваемого целочисленного набора $\left(x_{1}, \ldots, x_{m}, y_{1}, \ldots, y_{n}\right)$ с условием $(31)$, такого, что

$$
\left|x_{3}\right|+\cdots+\left|x_{m}\right|+\left|y_{1}\right|+\cdots+\left|y_{n}\right| \neq 0
$$

при каждом $j, 1 \leqslant j \leqslant n$, имеем

$$
\left(\theta_{j}^{1}, \theta_{j}^{3}, \ldots, \theta_{j}^{m}\right) \notin \mathscr{J}_{\nu}\left(x_{1}, x_{2}, \ldots, x_{m} ; y_{j}\right)
$$

где

$$
\begin{aligned}
\mathscr{J}_{\nu}\left(x_{1}, x_{2}, \ldots, x_{m} ; y_{j}\right)=\{ & \left(\theta_{j}^{1}, \theta_{j}^{3}, \ldots, \theta_{j}^{m}\right) \in[0,1]^{m-1}: \\
& \left.\left|\left(x_{1}-\xi x_{2}\right) \theta_{j}^{1}+x_{3} \theta_{j}^{3}+\cdots+x_{m} \theta_{j}^{m}+y_{j}\right| \leqslant \frac{1}{q_{\nu+1}}\right\} .
\end{aligned}
$$

Заметим, что для меры Лебега последнего множества выполнено неравенство

$$
\mu\left(\mathscr{J}_{\nu}\left(x_{1}, x_{2}, \ldots, x_{m} ; y_{j}\right)\right) \leqslant \frac{m^{3 / 2}}{q_{\nu+1}\left(\left|x_{1}-\xi x_{2}\right|+\left|x_{3}\right|+\cdots+\left|x_{m}\right|\right)} .
$$

Кроме того, если $x_{3}=\cdots=x_{m}=0$, то из (32) получаем, что некоторое $y_{j}$ не равно 0. Тогда, если $\left|x_{1}-\xi x_{2}\right|<1 / 2$, то (30) выполнено автоматически и, стало быть, можно считать, что

$$
\left|x_{1}-\xi x_{2}\right| \geqslant \frac{1}{2}
$$

Итак, для выполнения условия (30) достаточно, чтобы

$$
\left(\begin{array}{cccc}
\theta_{1}^{1} & \theta_{1}^{3} & \ldots & \theta_{1}^{m} \\
\ldots \ldots & \ldots \ldots & \ldots \\
\theta_{n}^{1} & \theta_{n}^{3} & \ldots & \theta_{n}^{m}
\end{array}\right) \notin \mathscr{J}_{\nu}=J_{\nu}^{(1)} \cup J_{\nu}^{(2)}
$$

где

$$
\begin{gathered}
J_{\nu}^{(1)}=\bigcup_{1} \bigcup_{2} J_{\nu}(x_{1}, x_{2}, \underbrace{0, \ldots, 0}_{m-2} ; y_{1}) \times \cdots \times J_{\nu}(x_{1}, x_{2}, \underbrace{0, \ldots, 0}_{m-2} ; y_{n}), \\
J_{\nu}^{(2)}=\bigcup_{3} \bigcup_{4} \bigcup_{5} J_{\nu}\left(x_{1}, x_{2}, x_{3}, \ldots, x_{m} ; y_{1}\right) \times \cdots \times J_{\nu}\left(x_{1}, x_{2}, x_{3}, \ldots, x_{m} ; y_{n}\right)
\end{gathered}
$$


и объединения $\bigcup_{i}, i=1, \ldots, 5$, берутся по следующим областям:

$$
\begin{array}{ll}
\bigcup_{1}: & \text { по }\left(x_{1}, x_{2}\right) \text { таким, что } 0<\max \left\{\left|x_{1}\right|,\left|x_{2}\right|\right\} \leqslant q_{\nu+1},\left|x_{1}-\xi x_{2}\right| \geqslant \frac{1}{2} ; \\
\bigcup_{2}: & \text { по }\left(y_{1}, \ldots, y_{n}\right) \text { таким, что } \max _{1 \leqslant j \leqslant n}\left|y_{j}\right| \leqslant 4\left|x_{1}-\xi x_{2}\right| ; \\
\bigcup_{3}: & \text { по }\left(x_{1}, x_{2}\right) \text { таким, что } 0<\max \left\{\left|x_{1}\right|,\left|x_{2}\right|\right\} \leqslant q_{\nu+1} ; \\
\bigcup_{4}: & \text { по }\left(x_{3}, \ldots, x_{m}\right) \text { таким, что } 0<\max _{3 \leqslant i \leqslant m}\left|x_{i}\right| \leqslant q_{\nu+1} ; \\
\bigcup_{5}: & \text { по }\left(y_{1}, \ldots, y_{n}\right) \text { таким, что } \max _{1 \leqslant j \leqslant n}\left|y_{j}\right| \leqslant 2\left(\left|x_{1}-\xi x_{2}\right|+\sum_{i=3}^{m}\left|x_{i}\right|\right) .
\end{array}
$$

Из (33) получаем, что

$$
\mu\left(J_{\nu}^{(1)}\right) \ll q_{\nu+1}^{2-n}, \quad \mu\left(J_{\nu}^{(2)}\right) \ll q_{\nu+1}^{m-n}, \quad \mu\left(\mathscr{J}_{\nu}\right) \ll q_{\nu+1}^{m-n} \leqslant q_{\nu+1}^{-1} .
$$

Ряд $\sum_{\nu=1}^{\infty} \frac{1}{q_{\nu}}$ сходится, и теорема 10, таким образом, вытекает из леммы Бореля-Кантелли.

В заключение этого пункта отметим, что при $m=n=2$ пример правильной матрицы $\Theta$, состоящей из чисел, линейно независимых вместе с единицей над $\mathbb{Z}$, и такой, что $R(\Theta)=2$, автору неизвестен.

2.3. Вырождение размерности, $R(\Theta)=3$. Приведенные в предыдущем пункте результаты показывают, насколько точна нижняя оценка пункта (i) теоремы 7 при $m \geqslant 2$. Ниже мы установим, что нижняя оценка из утверждения (iii) теоремы 7 тоже точна. Для этого мы будем использовать сингулярные матрицы.

Сначала рассмотрим весьма специальный случай.

Пусть числа $\xi^{1}, \xi^{2} \in(0,1 / 2)$ линейно независимы вместе с единицей над $\mathbb{Z}$. Пусть $\left(x_{1, \nu}, x_{2, \nu}, x_{3, \nu}\right)$ есть “расширенный” вектор наилучших приближений для набора $\left(\xi^{1}, \xi^{2}\right)$ (тут мы используем нестандартные для настоящей статьи обозначения, но это будет удобно). Тогда

$$
\left\|x_{1, \nu} \xi^{1}+x_{2, \nu} \xi^{2}\right\|=\left|x_{1, \nu} \xi^{1}+x_{2, \nu} \xi^{2}+x_{3, \nu}\right|<M_{\nu+1}^{-2}
$$

где можно считать, что

$$
M_{\nu}=\max _{i=1,2,3}\left|x_{i}\right|
$$

Положим

$$
\theta_{j}^{1}=\xi^{1} \theta_{j}^{3}, \quad \theta_{j}^{2}=\xi^{2} \theta_{j}^{3}, \quad 1 \leqslant j \leqslant n,
$$

и рассмотрим матрицу

$$
\left(\begin{array}{ccc}
\theta_{1}^{1} & \theta_{1}^{2} & \theta_{1}^{3} \\
\ldots \ldots & \ldots \ldots \\
\theta_{n}^{1} & \theta_{n}^{2} & \theta_{n}^{3}
\end{array}\right)=\left(\begin{array}{ccc}
\xi^{1} \theta_{1}^{3} & \xi^{2} \theta_{1}^{3} & \theta_{1}^{3} \\
\ldots \ldots \ldots \ldots \ldots & \ldots \\
\xi^{1} \theta_{n}^{3} & \xi^{2} \theta_{n}^{3} & \theta_{n}^{3}
\end{array}\right)
$$


TЕOPЕMA 11. Пусть ряд

$$
\sum_{\nu=1}^{\infty} M_{\nu+1}^{3}\left\|x_{1, \nu} \xi^{1}+x_{2, \nu} \xi^{2}\right\|^{n}
$$

сходится. Тогда для почти всех наборов $\left(\theta_{1}^{3}, \ldots, \theta_{n}^{3}\right) \in \mathbb{R}^{n}$ таких, что $0<$ $\theta_{2}^{3}, \ldots, \theta_{n}^{3}<1 / 2<\theta_{1}^{3}<1$, последовательность наилучших приближений для матрицы $\Theta$, определенной в (34), отличается от последовательности

$$
(x_{1, \nu}, x_{2, \nu}, x_{3, \nu}, \underbrace{0, \ldots, 0}_{n})
$$

не более чем на конечное число точек.

Доказательство теоремы 11 подобно доказательству теоремы 10: для почти всех $\left(\theta_{1}^{3}, \ldots, \theta_{n}^{3}\right) \in \mathbb{R}^{n}$ надо добиться выполнения неравенства

$$
\min _{x_{1}, x_{2}, x_{3} ; y_{1}, \ldots, y_{n}} \max _{1 \leqslant j \leqslant n}\left|\theta_{3}^{j}\left(x_{1} \xi^{1}+x_{2} \xi^{2}+x_{3}\right)+y_{j}\right|>\left|x_{1, \nu} \xi^{1}+x_{2, \nu} \xi^{2}+x_{3, \nu}\right|,
$$

где минимум берется по целочисленным векторам $\left(x_{1}, x_{2}, x_{3}, y_{1}, \ldots, y_{n}\right)$, отличным от

$$
\pm(x_{1, \nu}, x_{2, \nu}, x_{3, \nu}, \underbrace{0, \ldots, 0}_{n}), \quad \pm(x_{1, \nu+1}, x_{2, \nu+1}, x_{3, \nu+1}, \underbrace{0, \ldots, 0}_{n})
$$

и таким, что

$$
0 \neq \max _{i=1,2,3}\left|x_{i}\right| \leqslant M_{\nu+1}
$$

Естественно, следует различать случаи $\mathbf{y}=\mathbf{0}$ и $\mathbf{y} \neq \mathbf{0}$.

Из сходимости ряда $\sum_{\nu} M_{\nu}^{-1}$ (эта сходимость обеспечивается экспоненциальным ростом величины $M_{\nu}$; см., например, лемму из работы [34]) получаем следующее утверждение.

СлеДСтвИЕ. Если $n \geqslant 2$, то для почти всех наборов $\left(\theta_{1}^{3}, \ldots, \theta_{n}^{3}\right) \in \mathbb{R}^{n}$ для матрицы (34) выполнено $R(\Theta)=3$.

Теперь мы сформулируем и докажем утверждение, которое обобщает теорему Н. Г. Мощевитина и О.Н. Германа [34], которая, в свою очередь, обобщает результат автора [35]. Отметим, что в работе [34] анонсировано еще несколько результатов о существовании сингулярных систем специального вида (при $n=1)$, подробное доказательство которых пока не опубликовано. Формулировку одного из них мы приведем в разделе 12 (теорема 69).

Пусть $m^{*}>m$. Наряду с матрицей $\Theta$ вида (1) рассмотрим "расширенную" матрицу

$$
\Theta^{*}=\left(\begin{array}{cccccc}
\theta_{1}^{1} & \ldots & \theta_{1}^{m} & \theta_{1}^{m+1} & \ldots & \theta_{1}^{m^{*}} \\
\ldots \ldots \ldots \ldots \ldots \ldots \ldots \ldots \ldots \ldots & \ldots \ldots \ldots \\
\theta_{n}^{1} & \ldots & \theta_{n}^{m} & \theta_{n}^{m+1} & \ldots & \theta_{n}^{m^{*}}
\end{array}\right)
$$

с вещественными элементами. Таким образом, мы увеличили матрицу $\Theta$, добавив $n\left(m^{*}-m\right)$ элементов. Набор добавленных элементов можно отождествить с некоторым вектором $\underline{\Theta}=\left(\theta_{1}^{m+1}, \ldots, \theta_{n}^{m^{*}}\right)$ в пространстве $\mathbb{R}^{n\left(m^{*}-m\right)}$. Справедливо следующее утверждение, непосредственно обобщающее результат из [34] и развивающее подход из [35], [33]. 
TEOPEMA 12. Пусть точки

$$
\mathbf{z}_{\nu}=\left(x_{1, \nu}, \ldots, x_{m, \nu}, y_{1, \nu}, \ldots, y_{n, \nu}\right) \in \mathbb{Z}^{m+n}, \quad \nu=1,2,3, \ldots,
$$

суть все наилучшие приближения для матрицы $\Theta$. Пусть также величины $M_{\nu}$ и $\zeta_{\nu}$ суть элементы последовательностей (10), (11). Рассмотрим последовательность иелых точек

$$
\mathbf{z}_{\nu}^{*}=(x_{1, \nu}, \ldots, x_{m, \nu}, \underbrace{0, \ldots, 0}_{m^{*}-m}, y_{1, \nu}, \ldots, y_{n, \nu}) \in \mathbb{Z}^{m^{*}+n} .
$$

Предположим, что ряд

$$
\sum_{\nu=1}^{\infty} M_{\nu+1}^{\max \left(m+n, m^{*}\right)}\left(\log M_{\nu+1}\right)^{\delta\left(m^{*}, m+n\right)} \zeta_{\nu}^{n}, \quad \delta(a, b)= \begin{cases}1, & a=b, \\ 0, & a \neq b\end{cases}
$$

сходится. Тогда для почти всех"добавляемых" векторов $\underline{\Theta} \in \mathbb{R}^{n\left(m^{*}-m\right)}$ (в смьсле меры Лебега) последовательность наилучших приближений для матрииљ $\Theta^{*}$ отличается от последовательности $\mathbf{z}_{\nu}^{*}$ на не более чем конечное число точек.

Из теоремы 12 в свете предложения 1 непосредственно получаем следующее утверждение.

СлЕДСТВИЕ. Пусть $m \geqslant 2$, и пусть матрица $\Theta$ является $\psi$-сингулярной. Пусть также ряд

$$
\sum_{\nu=1}^{\infty} M_{\nu}^{\max \left(m+n, m^{*}\right)}\left(\log M_{\nu}\right)^{\delta\left(m^{*}, m+n\right)}\left(\psi\left(M_{\nu}\right)\right)^{n}
$$

сходится. Тогда для почти всех"добавляемых" векторов $\underline{\Theta} \in \mathbb{R}^{n\left(m^{*}-m\right)}$ последовательность всех наилучших приближений для матрищъ $\Theta^{*}$ отличается от последовательности (36) на не более чем конечное число точек.

Естественно, если положить $n=1, m=2, \psi(t)=t^{-m^{*}-2}$ и взять числа $\theta_{j}^{1}, \theta_{j}^{2}$, существование которых обеспечивается теоремой 1 , то наше следствие превращается в следующий результат из [35] и [33].

ТЕОрема 13. При $n=1$ и каждом $m \geqslant 2$ существует набор $\Theta$, состоящий из алгебраччески независимых чисел $\theta^{i}, 1 \leqslant i \leqslant m$, такой, что векторы наилучших приближений $\mathbf{z}_{\nu}$ при всех достаточно больших значениях $\nu$ лежат в некотором трехмерном подпространстве; т.е. $R(\Theta)=3$.

При произвольном $n$ и $m \geqslant 3$ из теорем 11 и 12 получаем следующий результат.

ТЕОРема 14. При $m \geqslant 3$ и каждом $n$ существует матрица $\Theta$, состоящая из линейно независимых вместе с единицей над $\mathbb{Z}$ чисел $\theta_{j}^{i}$, такая, что векторы наилучших приближений $\mathbf{z}_{\nu}$ при всех достаточно больших значениях $\nu$ лежат в некотором трехмерном подпространстве, т.е. $R(\Theta)=3$. 
ДокАЗАТЕЛЬСтво теоремы 12. Можно считать, что $\theta_{j}^{i} \in[0,1]$ при всех значениях $i, j$.

Нам надо доказать, что при фиксированной матрице $\Theta$, удовлетворяющей условию теоремы, для почти каждого “дополняющего" набора $\underline{\Theta}$ существует такое $\nu_{0}$, что для всех $\nu \geqslant \nu_{0}$

$$
\min \max _{1 \leqslant j \leqslant n}\left|\sum_{1 \leqslant i \leqslant m^{*}} x_{i} \theta_{j}^{i}+y_{j}\right|>\zeta_{\nu},
$$

где минимум берется по всем целым точкам

$$
\mathbf{z}=\left(x_{1}, \ldots, x_{m^{*}}, y_{1, \nu}, \ldots, y_{n}\right) \in \mathbb{Z}^{m^{*}+n} \backslash\{\mathbf{0}\}
$$

таким, что

$$
\max _{1 \leqslant j \leqslant m^{*}}\left|x_{j}\right| \leqslant M_{\nu+1}, \quad \mathbf{z} \neq \mathbf{z}_{\nu}^{*}
$$

Условие (39) будет выполнено, если для каждого вектора

$$
\left(x_{1}, \ldots, x_{m^{*}}, y_{1}, \ldots, y_{n}\right) \in \mathbb{Z}^{m^{*}+n} \backslash\left\{\mathbf{0}, \pm \mathbf{z}_{\nu}, \pm \mathbf{z}_{\nu+1}\right\}
$$

такого, что $\max _{1 \leqslant i \leqslant m^{*}}\left|x_{i}\right| \leqslant M_{\nu+1}$, имеет место следующее: для некоторого $j$ из промежутка $1 \leqslant j \leqslant n$ выполнено

$$
x_{m+1} \theta_{j}^{m+1}+\cdots+x_{m^{*}} \theta_{j}^{m^{*}} \notin J_{\nu}\left(y_{j}, x_{1}, \ldots, x_{m}\right),
$$

где

$J_{\nu}\left(y_{j}, x_{1}, \ldots, x_{m}\right)=\left(-y_{j}-x_{1} \theta_{j}^{1}-\cdots-x_{m} \theta_{j}^{m}-\zeta_{\nu},-y_{j}-x_{1} \theta_{j}^{1}-\cdots-x_{m} \theta_{j}^{m}+\zeta_{\nu}\right)$.

Условие (40) означает, что расстояние от точки $\left(\theta_{j}^{m+1}, \ldots, \theta_{j}^{m^{*}}\right) \in[0,1]^{m^{*}-m}$ до подпространства

$\left\{\left(u_{m+1}, \ldots, u_{m^{*}}\right) \in \mathbb{R}^{m^{*}-m}: x_{m+1} u_{m+1}+\cdots+x_{m^{*}} u_{m^{*}}=-y_{j}-x_{1} \theta_{j}^{1}-\cdots-x_{m} \theta_{j}^{m}\right\}$ не меньше, чем $\zeta_{\nu} \cdot\left(x_{m+1}^{2}+\cdots+x_{m^{*}}^{2}\right)^{-1 / 2}$.

Положим

$$
\begin{aligned}
\Omega_{\nu}(\mathbf{z})=\Omega_{\nu}(\mathbf{x}, \mathbf{y})=\left\{\underline{\Theta} \in[0,1]^{n\left(m^{*}-m\right)}:\right. & \\
& \left.x_{m+1} \theta_{j}^{m+1}+\cdots+x_{m^{*}} \theta_{j}^{m^{*}} \notin J_{\nu}\left(y_{j}, x_{1}, \ldots, x_{m}\right), 1 \leqslant j \leqslant n\right\}
\end{aligned}
$$

и

$$
\Omega_{\nu}=\bigcup_{\mathbf{y}} \bigcup_{\mathbf{x}} \Omega_{\nu}(\mathbf{x}, \mathbf{y}) .
$$

При этом в последней формуле объединения берутся по областям целых точек

$$
\left\{\mathbf{y}: \max _{1 \leqslant j \leqslant n}\left|y_{j}\right| \leqslant\left(m^{*}+1\right) M_{\nu+1}\right\}, \quad\left\{\mathbf{x}: 0<\max _{1 \leqslant i \leqslant m^{*}}\left|x_{i}\right| \leqslant M_{\nu+1}\right\} .
$$

Согласно лемме Бореля-Кантелли, для доказательства теоремы достаточно убедиться в том, что

$$
\sum_{\nu \geqslant \nu_{0}} \mu\left(\Omega_{\nu}\right) \rightarrow 0, \quad \nu_{0} \rightarrow+\infty
$$


Ho

$$
\begin{aligned}
\mu\left(\Omega_{\nu}\right) & \ll \zeta_{\nu}^{n} M_{\nu+1}^{n} \sum_{x_{1}, \ldots, x_{m}} \sum_{x_{m+1}, \ldots, x_{m^{*}}} \frac{1}{\left(\max _{m \leqslant i \leqslant m^{*}}\left|x_{i}\right|\right)^{n}} \\
& \ll \zeta_{\nu}^{n} M_{\nu+1}^{n+m} \sum_{1 \leqslant t \leqslant M_{\nu+1}} t^{m^{*}-m-n-1} \ll \zeta_{\nu}^{n} M_{\nu+1}^{\max \left(m+n, m^{*}\right)}\left(\log M_{\nu+1}\right)^{\delta\left(m^{*}, m+n\right)}
\end{aligned}
$$

Теперь соотношение (41) следует из сходимости ряда (37), и теорема доказана.

\section{3. Одномерные диофантовы приближения}

Обсудим простейшую ситуацию, когда $m=n=1$. В этом случае мы имеем дело с задачей о приближении одного числа $\alpha=\theta_{1}^{1}$ рациональными дробями.

3.1. Цепные дроби. Хорошо известно, что вопрос о наилучших приближениях в этом случае полностью решается посредством аппарата цепных дробей (см. [36]). Напомним, что если вещественное $\alpha$ представлено в виде цепной дроби

$$
\alpha=\left[a_{0} ; a_{1}, a_{2}, \ldots, a_{t}, \ldots\right]=a_{0}+\frac{1}{a_{1}+\frac{1}{a_{2}+\cdots+\frac{1}{a_{t}+\cdots}}},
$$

$a_{0} \in \mathbb{Z}, a_{t} \in \mathbb{N}, t=1,2,3, \ldots$ (конечной или бесконечной, в зависимости от того, является $\alpha$ рациональным числом или нет), то подходящими дробями к $\alpha$ называются рациональные дроби вида

$$
\frac{p_{\nu}}{q_{\nu}}=\left[a_{0} ; a_{1}, a_{2}, \ldots, a_{\nu}\right]=a_{0}+\frac{1}{a_{1}+\frac{1}{a_{2}+\cdots+\frac{1}{a_{\nu}}}} .
$$

Наилучшие приближения, определенные выше в п. 1.3 (точнее "расширенные" векторы $\mathbf{z}_{\nu} \in \mathbb{Z}^{2}$ ), будут (в терминологии книги [36]) наилучшими приближениями второго рода. Для них справедливо следующее утверждение (см. [36; теорема 16]).

ТеОрема 15. Всякое наилучшее приближение $\mathbf{z}_{\nu}$ имеет вид $\mathbf{z}_{\nu}=\left(q_{\nu}, p_{\nu}\right)$, где $q_{\nu}$ и $p_{\nu}$ являются знаменателем и числителем некоторой подходящей $\kappa \alpha$ дроби.

Отметим, что если $\alpha \neq p_{\nu} / q_{\nu}$ (в частности, если $\alpha$ есть число иррациональное), то для его приближения подходящей дробью имеют место неравенства (см. [36; теоремы 9, 13])

$$
\frac{1}{q_{\nu}\left(q_{\nu}+q_{\nu+1}\right)}<\left|\alpha-\frac{p_{\nu}}{q_{\nu}}\right|<\frac{1}{q_{\nu} q_{\nu+1}} .
$$

В частности,

$$
\left\|q_{\nu} \alpha\right\|>\frac{1}{2 q_{\nu+1}} .
$$


Или, на языке обозначений п. 1.3 (и при $\alpha \in(0,1)$ ),

$$
\zeta_{\nu} \geqslant \frac{1}{2 M_{\nu+1}} \quad \forall \nu \in \mathbb{N}
$$

Пользуясь случаем, отметим, что для разности из (42) имеется простое и изящное равенство

$$
\left|\alpha-\frac{p_{\nu}}{q_{\nu}}\right|=\frac{1}{q_{\nu}^{2}\left(\alpha_{\nu+1}+\alpha_{\nu}^{*}\right)},
$$

где

$$
\alpha_{\nu+1}=\left[a_{\nu+1} ; a_{\nu+2}, a_{\nu+3}, \ldots\right], \quad \alpha_{\nu}^{*}=\left[0 ; a_{\nu}, \ldots, a_{1}\right] .
$$

Это равенство позволяет провести весьма детальное исследование одномерных диофантовых приближений, например, свести основные задачи изучения сnектра Лагранжа

$$
\mathbb{L}=\left\{\lambda \in \mathbb{R}: \text { существует } \alpha \in \mathbb{R} \text { такое, что } \lambda=\left(\liminf _{q \rightarrow+\infty} q\|q \alpha\|\right)^{-1}\right\}
$$

к задачам о бесконечных последовательностях [37]. О спектре Лагранжа $\mathbb{L}$ имеется много работ - см., например, библиографию из книги [37] и замечательный обзор [38]. Никакой многомерный аналог равенства (45), по-видимому, неизвестен.

Если теперь предположить, что $\alpha$ иррационально и представляет собой сингулярную систему с $m=n=1$, то при всех достаточно больших $t$ согласно предложению 1 будет выполняться $\zeta_{\nu}<1 /\left(2 M_{\nu+1}\right)$, что противоречит $(44)$. Значит, иррациональных $\theta$, являющихся сингулярными системами, не бывает.

Равенство (45) естественно переписать в виде

$$
q_{\nu}\left\|q_{\nu} \alpha\right\|=\frac{1}{\alpha_{\nu+1}+\alpha_{\nu}^{*}} .
$$

Следует сказать, что имеет место (см., например, [39]) похожее равенство

$$
q_{\nu+1}\left\|q_{\nu} \alpha\right\|=\frac{1}{1+\frac{1}{\alpha_{\nu+2} \alpha_{\nu+1}^{* *}}},
$$

где

$$
\alpha_{\nu+1}^{* *}=a_{\nu+1}+\alpha_{\nu}^{*}=\left[a_{\nu+1} ; a_{\nu}, \ldots, a_{1}\right]
$$

Это равенство, в частности, позволяет исследовать спектр Дирихле

$$
\mathbb{D}=\left\{\lambda \in \mathbb{R}: \text { существует } \alpha \in \mathbb{R} \text { такое, что } \lambda=\left(\limsup _{q \rightarrow+\infty} q\|q \alpha\|\right)^{-1}\right\} .
$$

В отличие от спектра Лагранжа, изучению спектра Дирихле посвящено мало работ (см. библиографию в статье [40]).

Естественно, что определения спектров Лагранжа и Дирихле можно давать в терминах функции $\psi_{\alpha}(t)$. 
3.2. Функция $\psi_{\alpha}(t)$. Для случая $m=n=1$ при $\theta_{1}^{1}=\alpha$ функция $(20)$ принимает вид

$$
\psi_{\alpha}(t)=\min _{1 \leqslant x \leqslant t}\|\alpha x\| .
$$

Недавно И. Д. Кан и Н. Г. Мощевитин [41] получили следующий результат.

Теорема 16. Для произвольных двух иррациональных чисел $\alpha, \beta$ таких, что $\alpha \pm \beta \notin \mathbb{Z}$, функиия разности

$$
\psi_{\alpha}(t)-\psi_{\beta}(t)
$$

бесконечно много раз меняет знак при $t \rightarrow+\infty$.

Доказательство этой теоремы мы здесь не приводим. Отметим лишь, что оно связано с применением формул (45), (46).

Отметим также, что теоремы 1, 2 А.Я. Хинчина, процитированные нами в п. 1.2, показывают, что результат теоремы 16 не может быть перенесен на бо́льшие размерности.

Возьмем в теореме $1 \psi(t)=o\left(t^{-2}\right), t \rightarrow+\infty$. Пусть $\theta^{1}, \theta^{2}-$ те числа, существование которых утверждает теорема 1 . Возьмем другие числа $\left(\beta^{1}, \beta^{2}\right)$ плохо приближаемыми (в смысле линейной формы):

$$
\inf _{\left(x_{1}, x_{2}\right) \in \mathbb{Z}^{2} \backslash\{(0,0)\}}\left(\left\|x_{1} \beta^{1}+x_{2} \beta^{2}\right\|\left(\max \left\{\left|x_{1}\right|,\left|x_{2}\right|\right\}\right)^{2}\right)>0 .
$$

При достаточно больших $t$ выполняется неравенство

$$
\psi_{\left(\theta^{1}, \theta^{2}\right)}(t)<\psi_{\left(\beta^{1}, \beta^{2}\right)}(t)
$$

Ситуация для совместных приближений аналогична. Надо взять в теореме 2 функцию $\psi_{1}(t)=o\left(t^{-1 / 2}\right), t \rightarrow+\infty$, числа $\theta_{1}, \theta_{2}$ из теоремы 2 , а также совместно плохо приближаемые числа $\left(\begin{array}{c}\beta_{1} \\ \beta_{2}\end{array}\right)$ :

$$
\inf _{x \in \mathbb{Z} \backslash\{0\}}\left(\max _{j=1,2}\left\|x \beta_{j}\right\| \cdot|x|^{1 / 2}\right)>0 \text {. }
$$

Тогда

$$
\psi_{\left(\begin{array}{c}
\theta_{1} \\
\theta_{2}
\end{array}\right)}(t)<\psi_{\left(\begin{array}{c}
\beta_{1} \\
\beta_{2}
\end{array}\right)}^{(t)}
$$

для всех достаточно больших $t$.

3.3. Двумерные решетки. Здесь мы хотим обобщить неравенства (42) (а значит, и оценку снизу (44)) удобным для приложений образом.

Рассмотрим двумерное вполне рациональное линейное подпространство $\pi \subset \mathbb{R}^{d}$ и двумерную решетку $\Lambda=\pi \cap \mathbb{Z}^{d}$, в нем содержащуюся. Будем считать, что множество

$$
\mathscr{B}=\pi \cap\left\{\mathbf{z}=(\mathbf{x}, \mathbf{y}): \max _{1 \leqslant i \leqslant m}\left|x_{i}\right| \leqslant 1\right\}
$$

компактно. Тогда sup-норма на пространстве векторов х задает на двумерном

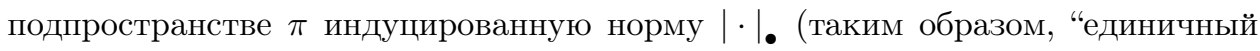


шар" $\mathscr{B}=\left\{\mathbf{z} \in \pi:|\mathbf{z}|_{\bullet}=1\right\}$ есть ограниченное выпуклое центрально-симметричное множество). Рассмотрим одномерное подпространство $\ell \subset \pi$. Пусть 0 единственная точка решетки $\Lambda$, которая лежит в $\ell$, и, более того,

$$
\ell \cap\left\{\mathbf{z}=\left(\mathbf{x}^{\prime}, \mathbf{y}^{\prime}\right): x_{1}^{\prime}=\cdots=x_{m}^{\prime}=0\right\}=\{\mathbf{0}\} .
$$

Выберем некоторое линейное подпространство $\mathscr{L}$ такое, что

$$
\mathscr{L} \cap \pi=\ell, \quad \operatorname{dim} \mathscr{L} \geqslant 1 .
$$

Поскольку $\ell$ не является вполне рациональным, то $\mathscr{L}$ тоже не является вполне рациональным.

Определим наилучшее приближение точками решетки $\Lambda$ подпространства $\ell$ относительно нормы $|\cdot|$ и подпространства $\mathscr{L}$ как такую точку $\mathbf{z}=(\mathbf{x}, \mathbf{y}) \in \Lambda$, что

$$
\operatorname{dist}(\mathbf{z}, \mathscr{L})=\min \operatorname{dist}\left(\mathbf{z}^{\prime}, \mathscr{L}\right),
$$

где минимум берется по всем точкам $\mathbf{z}^{\prime} \in \Lambda$ таким, что

$$
0<\left|\mathbf{z}^{\prime}\right|_{\bullet} \leqslant|\mathbf{z}|_{\bullet}
$$

и $\operatorname{dist}(\mathbf{z}, \mathscr{L})$ обозначает расстояние в $\operatorname{sup-норме~}\left(\right.$ в $\left.\mathbb{R}^{d}\right)$ от точки $\mathbf{z}$ до подпространства $\mathscr{L}$.

Естественно, наилучшие приближения должны располагаться в виде бесконечных последовательностей

$$
\begin{gathered}
\pm \mathbf{z}_{1}, \pm \mathbf{z}_{2}, \ldots, \pm \mathbf{z}_{\nu}, \pm \mathbf{z}_{\nu+1}, \ldots \\
\operatorname{dist}\left(\mathbf{z}_{1}, \mathscr{L}\right)>\operatorname{dist}\left(\mathbf{z}_{2}, \mathscr{L}\right)>\cdots>\operatorname{dist}\left(\mathbf{z}_{\nu}, \mathscr{L}\right)>\operatorname{dist}\left(\mathbf{z}_{\nu+1}, \mathscr{L}\right)>\cdots \\
\left|\mathbf{z}_{1}\right|_{\bullet}<\left|\mathbf{z}_{2}\right|_{\bullet}<\cdots<\left|\mathbf{z}_{\nu}\right|_{\bullet}<\left|\mathbf{z}_{\nu+1}\right|_{\bullet}<\cdots
\end{gathered}
$$

аналогичных последовательностям (10), (11). Строгая монотонность величин $\operatorname{dist}\left(\mathbf{z}_{\nu}, \mathscr{L}\right),\left|\mathbf{z}_{\nu}\right|$ • определяется постановкой задачи. Будем говорить, что набор $(\pi, \ell, \mathscr{L})$ является правилъным, если для каждого достаточно большого значения индекса $\nu$ целочисленный вектор $\mathbf{z}_{\nu} \in \Lambda$ определен однозначно с точностью до знака.

Очевидным обобщением неравенств (42) является следующее утверждение.

ПреДЛОЖЕНИЕ 2. Если набор $(\pi, \ell, \mathscr{L})$ является правилъным, то с некоторыми положительными постоянными $C_{1}, C_{2}$, зависящими от $\pi, \ell u \mathscr{L}$, при всех $\nu$ выполняется

$$
C_{1} \leqslant \operatorname{dist}\left(\mathbf{z}_{\nu}, \mathscr{L}\right)\left|\mathbf{z}_{\nu+1}\right|_{\bullet} \leqslant C_{2}
$$

Чтобы убедиться в справедливости предложения 2, достаточно рассмотреть двумерную выпуклую центрально-симметричную область

$$
\left\{\mathbf{z} \in \pi:|\mathbf{z}|_{\bullet} \leqslant\left|\mathbf{z}_{\nu+1}\right|_{\bullet}, \operatorname{dist}(\mathbf{z}, \mathscr{L}) \leqslant \operatorname{dist}\left(\mathbf{z}_{\nu}, \mathscr{L}\right)\right\},
$$

на границе которой лежат две линейно независимые точки $\mathbf{z}_{\nu}, \mathbf{z}_{\nu+1} \in \Lambda$. 


\section{4. Сингулярные системы в задаче о совместных диофантовых приближениях}

Случай $m=1$ называется задачей о совместных диофантовых приближениях. Всюду ниже в настоящем разделе мы полагаем $m=1$ и используем обозначение $\theta_{j}^{1}=\theta_{j}, 1 \leqslant j \leqslant n$. В этом случае

$$
\mathbf{x}=x_{1}=x \in \mathbb{Z}^{1}, \quad M(\mathbf{x})=|x|, \quad L_{j}(x)=\theta_{j} x
$$

и речь идет о малости величины

$$
\max _{1 \leqslant j \leqslant n}\left\|x \theta_{j}\right\| .
$$

Пустые параллелепипеды (16), (17) теперь имеют вид:

$$
\begin{aligned}
\Omega_{\nu}^{(0)} & =\left\{\mathbf{z}^{\prime}=\left(x^{\prime}, y_{1}^{\prime}, \ldots, y_{n}^{\prime}\right):\left|x^{\prime}\right| \leqslant x_{\nu}, \max _{1 \leqslant j \leqslant n}\left|x^{\prime} \theta_{j}+y_{j}^{\prime}\right| \leqslant \max _{1 \leqslant j \leqslant n}\left\|x_{\nu} \theta_{j}\right\|\right\}, \\
\Omega_{\nu} & =\left\{\mathbf{z}^{\prime}=\left(x^{\prime}, y_{1}^{\prime}, \ldots, y_{n}^{\prime}\right):\left|x^{\prime}\right| \leqslant x_{\nu+1}, \max _{1 \leqslant j \leqslant n}\left|x^{\prime} \theta_{j}+y_{j}^{\prime}\right| \leqslant \max _{1 \leqslant j \leqslant n}\left\|x_{\nu} \theta_{j}\right\|\right\} .
\end{aligned}
$$

В первом из них нет других целых точек, кроме точек $\mathbf{0}, \pm \mathbf{z}_{\nu}$. Во втором нет других целых точек кроме $\mathbf{0}$ и $\pm \mathbf{z}_{\nu}, \pm \mathbf{z}_{\nu+1}$. Здесь $\mathbf{z}_{\nu}=\left(x_{\nu}, y_{1, \nu}, \ldots, y_{n, \nu}\right)$ и $x_{\nu}>0$.

Отметим, что для вещественнозначной функции $\psi(t)=o\left(t^{-1 / n}\right)$ набор вещественных чисел $\Theta \in \mathbb{R}^{n}$ будет $\psi$-сингулярным, если при всяком достаточно большом $t$ система диофантовых неравенств

$$
\max _{1 \leqslant j \leqslant n}\left\|x \theta_{j}\right\| \leqslant \psi(t), \quad 0<x \leqslant t,
$$

имеет целочисленное решение $x \in \mathbb{Z}$, или, что то же самое, при всех достаточно больших $\nu$ для знаменателей $x_{\nu}$ наилучших совместных приближений выполнено неравенство

$$
\max _{1 \leqslant j \leqslant n}\left\|x_{\nu} \theta_{j}\right\| \leqslant \psi\left(x_{\nu+1}\right)
$$

Напомним, что для вектора $\Theta=\left(\theta_{1}, \ldots, \theta_{n}\right) \in \mathbb{R}^{n}$ через $\operatorname{dim}_{\mathbb{Z}} \Theta$ мы обозначаем максимальное количество линейно независимых чисел из набора $1, \theta_{1}, \ldots, \theta_{n}$.

4.1. Независимость векторов наилучших приближений. Сейчас мы сформулируем в виде следствий утверждения, которые мгновенно выводятся из описанных выше результатов.

Из предложения 2 п. 3.3 получаем

СлеДСтвИЕ 1. Пусть $\operatorname{dim}_{\mathbb{Z}} \Theta=2$. Тогда с некоторой постоянной $\gamma=$ $\gamma(\Theta)>0$ выполнено

$$
\zeta_{\nu} \geqslant \frac{\gamma}{M_{\nu+1}} \quad \forall \nu \in \mathbb{N} .
$$

Из утверждения (ii) теоремы 7 и замечания в п. 2.1 (с. 56) получаем следующие утверждения. 
Следствие 2 . В случае $n \geqslant 2 u \operatorname{dim}_{\mathbb{Z}} \Theta \geqslant 3$ найдется бесконечно много значений $\nu$ таких, что векторы наилучших приближений $\mathbf{z}_{\nu}, \mathbf{z}_{\nu+1} u \mathbf{z}_{\nu+2}$ линейно независимы.

СлеДСтвиЕ 3. Eсли $n=2 u \operatorname{dim}_{\mathbb{Z}} \Theta=3$, mо существует бесконечная последовательность значений $\nu$ такая, что для векторов наилучших приближений $z_{\nu+i}=\left(x_{\nu+i}, y_{1, \nu+i}, y_{2, \nu+i}\right), i=0,1,2$, выполняется

$$
\operatorname{det}\left(\begin{array}{ccc}
x_{\nu} & y_{1, \nu} & y_{2, \nu} \\
x_{\nu+1} & y_{1, \nu+1} & y_{2, \nu+1} \\
x_{\nu+2} & y_{1, \nu+2} & y_{2, \nu+2}
\end{array}\right) \neq 0 .
$$

Следствие 3 имеется в явном виде у Дж. Лагариаса [30]. В неявном виде у В. Ярника (см. [13; с. 333-337]) фактически имеется следующее более общее утверждение.

СлЕДСтвиЕ 4. Пусть $n \geqslant 2 u \operatorname{dim}_{\mathbb{Z}} \Theta \geqslant 3$. Тогда найдутся два индекса $j_{1} \neq j_{2}$ и бесконечная последовательность значений $\nu$ такие, что для векторов наилучших приближений $z_{\nu+i}=\left(x_{\nu+i}, y_{1, \nu+i}, \ldots, y_{n, \nu+i}\right), i=0,1,2$, выполнено

$$
\operatorname{det}\left(\begin{array}{ccc}
x_{\nu} & y_{j_{1}, \nu} & y_{j_{2}, \nu} \\
x_{\nu+1} & y_{j_{1}, \nu+1} & y_{j_{2}, \nu+1} \\
x_{\nu+2} & y_{j_{1}, \nu+2} & y_{j_{2}, \nu+2}
\end{array}\right) \neq 0 .
$$

Формально в [13] присутствует чуть более слабое утверждение. Поясним, как получается следствие 4 (ср. с леммой на стр. 333 из [13]).

Согласно следствию 2 , для бесконечного множества значений индекса $\nu$ матрица

$$
\left(\begin{array}{cccc}
x_{\nu} & y_{1, \nu} & \ldots & y_{n, \nu} \\
x_{\nu+1} & y_{1, \nu+1} & \ldots & y_{n, \nu+1} \\
x_{\nu+2} & y_{1, \nu+2} & \ldots & y_{n, \nu+2}
\end{array}\right)
$$

имеет ранг 3. Если все определители (48) обращаются в нуль, то для некоторых $j_{1}, j_{2}, j_{3}$ имеем

$$
\operatorname{det}\left(\begin{array}{ccc}
y_{j_{1}, \nu} & y_{j_{2}, \nu} & y_{j_{3}, \nu} \\
y_{j_{1}, \nu+1} & y_{j_{2}, \nu+1} & y_{j_{3}, \nu+1} \\
y_{j_{1}, \nu+2} & y_{j_{2}, \nu+2} & y_{j_{3}, \nu+1}
\end{array}\right) \neq 0
$$

т. е. трехмерные векторы (столбцы матрицы (49))

$$
\left(\begin{array}{c}
y_{j_{1}, \nu} \\
y_{j_{1}, \nu+1} \\
y_{j_{1}, \nu+2}
\end{array}\right), \quad\left(\begin{array}{c}
y_{j_{2}, \nu} \\
y_{j_{2}, \nu+1} \\
y_{j_{2}, \nu+2}
\end{array}\right), \quad\left(\begin{array}{c}
y_{j_{3}, \nu} \\
y_{j_{3}, \nu+1} \\
y_{j_{3}, \nu+2}
\end{array}\right)
$$

линейно независимы. Но тогда получается, что вектор

$$
\left(\begin{array}{c}
x_{\nu} \\
x_{\nu+1} \\
x_{\nu+2}
\end{array}\right)
$$

линейно зависим с любыми двумя векторами из (50), чего не может быть. 
Прежде чем формулировать следствие 5, проведем некоторые рассуждения. Пусть $\operatorname{dim}_{\mathbb{Z}} \Theta \geqslant 3$. Рассмотрим определитель из следствия 4, не обращающийся в нуль при некотором значении параметра $\nu$. Тогда

$$
\begin{aligned}
1 & \leqslant\left|\operatorname{det}\left(\begin{array}{ccc}
x_{\nu} & y_{j_{1}, \nu} & y_{j_{2}, \nu} \\
x_{\nu+1} & y_{j_{1}, \nu+1} & y_{j_{2}, \nu+1} \\
x_{\nu+2} & y_{j_{1}, \nu+2} & y_{j_{2}, \nu+2}
\end{array}\right)\right| \\
& =\left|\operatorname{det}\left(\begin{array}{ccc}
x_{\nu} & y_{j_{1}, \nu}-\theta_{j_{1}} x_{\nu} & y_{j_{2}, \nu}-\theta_{j_{2}} x_{\nu} \\
x_{\nu+1} & y_{j_{1}, \nu+1}-\theta_{j_{1}} x_{\nu+1} & y_{j_{2}, \nu+1}-\theta_{j_{2}} x_{\nu+1} \\
x_{\nu+2} & y_{j_{1}, \nu+2}-\theta_{j_{1}} x_{\nu+2} & y_{j_{2}, \nu+2}-\theta_{j_{2}} x_{\nu+2}
\end{array}\right)\right| \\
& \leqslant 6 x_{\nu+2} \max _{1 \leqslant j \leqslant n}\left\|x_{\nu} \theta_{j}\right\| \max _{1 \leqslant j \leqslant n}\left\|x_{\nu+1} \theta_{j}\right\|=6 M_{\nu+2} \zeta_{\nu} \zeta_{\nu+1} .
\end{aligned}
$$

Таким образом, для бесконечно многих значений $\nu$ выполнено

$$
M_{\nu+2} \zeta_{\nu+1} \geqslant \frac{1}{6 \zeta_{\nu}}
$$

Очевидным становится следующее утверждение.

СлеДСтвиЕ 5. Пусть $\operatorname{dim}_{\mathbb{Z}} \Theta \geqslant 3$. Тогда

$$
\limsup _{\nu \rightarrow+\infty} M_{\nu+1} \zeta_{\nu}=+\infty
$$

Утверждение (52) имеется у В. Ярника в [9] при $n=2$ (см. [9; Satz 9]). В общем случае оно сформулировано в [14]. Отметим, что в [9] соотношение (52) доказывается с помощью соображений переноса. Точнее, В. Ярник везде доказывает равенство

$$
\limsup _{t \rightarrow+\infty} t \psi_{\Theta}(t)=+\infty
$$

которое является следствием соотношения (52). Здесь мы приводим более точный результат из работы автора [42] (в [42] автор не ссылается на работы В. Ярника, поскольку тогда он не знал об их существовании).

TEOPEMA 17. Пусть $n \geqslant 2 u \operatorname{dim}_{\mathbb{Z}} \Theta \geqslant 3$. Тогдa

$$
\zeta_{\nu} M_{\nu+1} \rightarrow+\infty, \quad \nu \rightarrow+\infty
$$

Доказательство. 1. Во-первых, отметим следующее. Пусть $\Lambda^{2} \in \mathbb{Z}^{n+1}-$ произвольная двумерная подрешетка и $\operatorname{det}_{2} \Lambda^{2}-$ ее двумерный фундаментальный объем. Тогда множество решеток

$$
\left\{\Lambda^{2} \subset \mathbb{Z}^{n+1}: \operatorname{det}_{2} \Lambda^{2} \leqslant \gamma\right\}
$$

конечно для любого положительного $\gamma$.

2. Во-вторых, если рассмотреть двумерную решетку $\Lambda_{\nu}^{2}=\left\langle\mathbf{z}_{\nu}, \mathbf{z}_{\nu+1}\right\rangle_{\mathbb{Z}}$, порожденную двумя последовательными наилучшими приближениями, то из условия $\operatorname{conv}\left(\mathbf{0}, \mathbf{z}_{\nu}, \mathbf{z}_{\nu+1}\right) \subset \Omega_{\nu}$ (область $\Omega_{\nu}$ определена в (47)) следует, что с некоторой положительной постоянной $C(\Theta)$ имеет место неравенство

$$
\frac{1}{2} \operatorname{det}_{2} \Lambda_{\nu}^{2}=\operatorname{vol}_{2}\left(\operatorname{conv}\left(\mathbf{0}, \mathbf{z}_{\nu}, \mathbf{z}_{\nu+1}\right)\right) \leqslant C(\Theta) \zeta_{\nu} M_{\nu+1}
$$


3. В-третьих, из условия $\operatorname{dim}_{\mathbb{Z}} \Theta \geqslant 3$ следует, что для каждого фиксированного $\mu$ при достаточно большом $\nu$ будет выполнено $\mathbf{z}_{\nu} \notin \Lambda_{\mu}^{2}$. Значит, при каждом $\mu$ и при достаточно большом $\nu$ двумерная решетка $\Lambda_{\nu}^{2}$ не будет совпадать ни с одной из решеток $\Lambda_{1}^{2}, \ldots, \Lambda_{\mu}^{2}$.

Теорема 17 вытекает из сказанного выше.

Далее мы формулируем следствие теоремы 17, в некотором смысле аналогичное предложению 2 из п. 3.3.

По аналогии с тем, что проделано в п. 3.3, рассмотрим вполне рациональное линейное подпространство $\pi \subset \mathbb{R}^{d}$ такое, что $\operatorname{dim} \pi \geqslant 3$ и пересечение $\pi \cap\left\{\mathbf{z}=(\mathbf{x}, \mathbf{y}): \max _{1 \leqslant i \leqslant m}\left|x_{i}\right| \leqslant 1\right\}$ ограничено, а также решетку $\Lambda=\pi \cap \mathbb{Z}^{d}$. Пусть снова $\ell \subset \pi$ - некоторое одномерное линейное подпространство в $\pi$. Но сейчас (в отличие от того, что было в п. 3.3) мы будем предполагать, что подпространство $\ell$ не лежит ни в каком собственном рациональном подпространстве подпространства $\pi$ (это нечто большее, чем то, что $\mathbf{0}$ есть единственная точка решетки $\Lambda$, лежащая в $\ell$ ).

Для индуцированной нормы $|\cdot|$. на $\pi$ рассмотрим последовательность наилучших приближений $\mathbf{z}_{\nu} \in \Lambda$ к подпространству $\ell$ относительно нормы $|\cdot|$, соответствующую подпространству $\mathscr{L} \supset \ell=\mathscr{L} \cap \pi$ (тут определение наилучших приближений и правильного набора $(\pi, \ell, \mathscr{L})$ совершенно аналогично определению, данному в п. 3.3).

ПреДЛОЖЕНИЕ 3. В описанных выше условиях для правилъного набора $(\pi, \ell, \mathscr{L})$ выполняется

$$
\operatorname{dist}\left(\mathbf{z}_{\nu}, \mathscr{L}\right)\left|\mathbf{z}_{\nu+1}\right|_{\bullet} \rightarrow+\infty, \quad \nu \rightarrow+\infty .
$$

4.2. Вырождение размерности наилучших совместных приближений. Сформулируем результат, полученный автором в [32] (см. также [33]), представляющий контрпример к предположению Дж. Лагариаса [30].

ТЕОРема 18. Пусть $n \geqslant 3$. Тогда найдутся числа $\theta_{1}, \ldots, \theta_{n}$, линейно независимые вместе с единищей над $\mathbb{Z}$ и такие, что среди любых $n+1$ идущих подряд векторов наилучших приближении $\mathbf{z}_{\nu}, \ldots, \mathbf{z}_{\nu+n}$ можно выбрать не более трех линейно независимых.

Прокомментируем вкратце доказательство этой теоремы. Числа $\theta_{1}, \ldots, \theta_{n}$ задаются с помощью рациональных приближений к ним. Сами рациональные приближения строятся индуктивно. Основным инструментом для осуществления индуктивного шага конструкции является следующее утверждение.

Лемма 1. Пусть для рационалъной точки $\alpha=\left(a_{1} / q, \ldots, a_{n} / q\right)$ такой, что $\left(a_{1}, \ldots, a_{n}, q\right)=1$, выполнено следующее:

(i) конечная последовательность совместных наилучших приближений

$$
\begin{gathered}
\mathbf{z}_{\nu}=\left(x_{\nu}, y_{1, \nu}, \ldots, y_{n, \nu}\right), \quad 1 \leqslant \nu \leqslant k \\
\left(x_{k}, y_{1, k}, \ldots, y_{n, k}\right)=\left(q, a_{1}, \ldots, a_{n}\right),
\end{gathered}
$$

определена однозначно;

(ii) найдутся двумерное вполне ращиональное подпространство $\pi$ и номер $\mu<k$ maкuе, чmо

$$
\mathbf{z}_{\nu} \in \pi, \quad \mu \leqslant \nu \leqslant k
$$


(iii) для наилучшего приближения с номером $k$ выполнено неравенство

$$
\zeta_{k}<\rho(\pi)
$$

(величина $\rho(\pi)$ определена в $(24)$ ).

Тогда найдется положительное $\varepsilon$ такое, что для всех точек $\beta$, лежащих в $\varepsilon$-окрестности точки $\alpha$, будет выполнено следующее:

$\left(\mathrm{i}^{*}\right)$ все векторь наилучших приближений $\kappa$ к будут наилучшими приближениями и $\kappa$ тоже;

(iі*) все расширенные векторы наилучших приближений $\kappa \beta$, знаменатель $x$ которых находится в промежутке $x_{\mu} \leqslant x \leqslant x_{k}$, лежат в подпространстве $\pi$.

Для осуществления индуктивного шага построения следует выбрать двумерное вполне рациональное подпространство $\pi_{1} \ni \alpha$, а затем новую рациональную точку $\alpha_{1} \in \pi_{1}$, лежащую близко к $\alpha$, так, чтобы она, в свою очередь, снова удовлетворяла условиям леммы 1 с заменой $\pi$ на $\pi_{1}$. Причем $\alpha_{1}$ следует выбирать так, чтобы для нее имелось $>n+1$ векторов наилучших приближений, лежащих в подпространстве $\pi_{1}$. Независимость вместе с единицей над $\mathbb{Z}$ компонент предельного вектора надо обеспечить посредством выбора двумерных подпространств таким образом, чтобы $R(\Theta)=n+1$.

\section{5. Сингулярность и диофантов тип}

Пусть функция $\varphi(t)$ монотонно убывает к нулю при $t \rightarrow+\infty$. Принято говорить, что набор $\Theta$ имеет диофантов тип $\leqslant \varphi(t)$, если для бесконечно многих $\mathbf{x} \in \mathbb{Z}^{m}$ имеет место неравенство

$$
\max _{1 \leqslant j \leqslant n}\left\|L_{j}(\mathbf{x})\right\| \leqslant \varphi\left(\max _{1 \leqslant i \leqslant m}\left|x_{i}\right|\right) .
$$

В работе [13] В. Ярник установил простые и замечательные оценки диофантова типа для сингулярных матриц. Ниже мы их приводим. Как показал М. Лоран [43], неравенства В. Ярника в случаях $(m, n)=(1,2),(2,1)$ неулучшаемы. Точную формулировку теоремы М. Лорана мы приводим в п. 8.3 нашей статьи. Ниже в пп. 5.1, 5.3, 5.5 мы приводим оригинальные результаты В. Ярника. В пп. 5.2, 5.4, 5.6 имеется усиление теоремы В. Ярника, принадлежащее автору и установленное в препринте [44].

5.1. Случай $m=1$. Сначала рассмотрим задачу о совместных приближениях. В. Ярник (см. [13; теорема 3]) получил следующий результат.

Теорема 19. Пусть $\psi(t)$ - непрерьвная убъвающая $\kappa$ нулю при $t \rightarrow+\infty$ функция вещественного переменного $t$, причем функиия $t \psi(t)$ монотонно возpастает $\kappa$ бесконечности при $t \rightarrow+\infty$. Обозначим через $\omega(t)$ функцию, обратную $\kappa t \psi(t)$. Положим

$$
\varphi^{[\psi]}(t)=\psi\left(\omega\left(\frac{1}{6 \psi(t)}\right)\right) .
$$

Пусть $n \geqslant 2$ u $\operatorname{dim}_{\mathbb{Z}} \Theta \geqslant 3$, а набор $\Theta$ является $\psi$-сингулярным. Тогда для бесконечного количества натуральных чисел $x$ выполнено

$$
\max _{1 \leqslant j \leqslant n}\left\|x \theta_{j}\right\| \leqslant \varphi^{[\psi]}(x) .
$$


Сейчас мы докажем более точный факт: если наилучшие приближения $\mathbf{z}_{\nu}$, $\mathbf{z}_{\nu+1} \quad$ и $\mathbf{z}_{\nu+2}$ линейно независимъ, то

$$
\zeta_{\nu+1} \leqslant \varphi^{[\psi]}\left(x_{\nu+1}\right)
$$

из которого теорема 19 вытекает в свете следствия 2 на с. 69.

Действительно, рассмотрим определитель (48), не обращающийся в нуль. Тогда из неравенства (51), используя предложение 1 на с. 52, получаем

$$
1 \leqslant 6 M_{\nu+2} \psi\left(M_{\nu+2}\right) \psi\left(M_{\nu+1}\right)=6 x_{\nu+2} \psi\left(x_{\nu+2}\right) \psi\left(x_{\nu+1}\right),
$$

откуда (55) сразу следует.

Чтобы пояснить результат теоремы 19, сформулируем следствие о диофантовых экспонентах, также принадлежащее В. Ярнику (см. [13; часть I теоремы 1]).

СлеДСтвиЕ. Пусть $n \geqslant 2 u \operatorname{dim}_{\mathbb{Z}} \Theta \geqslant 3$. Обозначим через $\alpha(\Theta)$ u $\beta(\Theta)$ супремумы тех $\gamma$, при которьх для функции Ярника (20) выполнено соответственно

$$
\begin{aligned}
& \limsup _{t \rightarrow+\infty} t^{\gamma} \psi_{\Theta}(t)<+\infty, \\
& \liminf _{t \rightarrow+\infty} t^{\gamma} \psi_{\Theta}(t)<+\infty
\end{aligned}
$$

(понятно, что $1 / n \leqslant \alpha(\Theta) \leqslant \beta(\Theta) \leqslant+\infty)$. Тогда, если $\alpha(\theta)<1$, то

$$
\beta(\Theta) \geqslant \frac{\alpha^{2}(\Theta)}{1-\alpha(\Theta)}
$$

если же $\alpha(\Theta)=1$, то $\beta(\Theta)=+\infty$.

5.2. Случай $m=1, n=3$. Для $\alpha \in[1 / 3,1]$ положим

$$
g_{1}(\alpha)=\frac{(1-\alpha) \alpha+\sqrt{(1-\alpha)^{2} \alpha^{2}+4 \alpha\left(2 \alpha^{2}-2 \alpha+1\right)}}{4 \alpha^{2}-4 \alpha+2} .
$$

Величина $g_{1}(\alpha)$ есть корень уравнения

$$
\left(2 \alpha^{2}-2 \alpha+1\right) x^{2}+\alpha(\alpha-1) x-\alpha=0 .
$$

Легко видеть, что

$$
\begin{gathered}
g_{1}(\alpha)=\max _{\delta \geqslant 1, \gamma(1-\alpha)-\alpha>0} \min \left\{\delta, \frac{\alpha}{\gamma(1-\alpha)-\alpha}, \frac{1}{\alpha}-\left(\frac{1}{\alpha}-1\right) \frac{\delta}{\gamma}\right\}, \\
g_{1}\left(\frac{1}{3}\right)=g_{1}(1)=1 .
\end{gathered}
$$

Более того, при $\alpha \in(1 / 3,1)$ выполняется неравенство $g_{1}(\alpha)>1$. 
Пусть $\alpha_{0} \in(1 / 2,1)$ есть корень уравнения

$$
x^{3}-x^{2}+2 x=1 .
$$

Тогда в интервале $1 / 3<\alpha<\alpha_{0}$ выполняется неравенство

$$
g_{1}(\alpha)>\max \left\{1, \frac{\alpha}{1-\alpha}\right\} .
$$

Теорема 20. Пусть $m=1, n=3$ и матрица $\Theta$ cocmoum из чисел $\theta_{1}$, $\theta_{2}, \theta_{3}$, линейно независимых вместе с единицей над $\mathbb{Z}$. Тогда

$$
\beta(\Theta) \geqslant \alpha(\Theta) g_{1}(\alpha(\Theta))
$$

Очевидно, что неравенство (60) сильнее, чем результат теоремы 19 (и ее следствия) в интервале $1 / 3<\alpha(\Theta)<\alpha_{0}$.

ДокАЗАТЕЛЬство. Будем предполагать, что неравенство $\psi_{\Theta}(t) \leqslant \psi(t)$ выполнено с некоторой непрерывной убывающей к нулю функцией $\psi(t)$. Также следует предположить, что функция $t \mapsto t \psi(t)$ монотонно возрастает к бесконечности при $t \rightarrow+\infty$.

Рассмотрим последовательность векторов наилучших приближений $\mathbf{z}_{\nu}=$ $\left(x_{\nu}, y_{1, \nu}, y_{2, \nu}, y_{3, \nu}\right)$. Из условия линейной независимости следует, что для бесконечной последовательности пар индексов $\nu<k$ при $\nu \rightarrow+\infty$ имеют место следующие три утверждения:

- обе тройки

$$
\left(\mathbf{z}_{\nu-1}, \mathbf{z}_{\nu}, \mathbf{z}_{\nu+1}\right), \quad\left(\mathbf{z}_{k-1}, \mathbf{z}_{k}, \mathbf{z}_{k+1}\right)
$$

состоят из линейно независимых векторов;

- имеется некоторое двумерное подпространство $\pi$ такое, что

$$
\mathbf{z}_{l} \in \pi, \quad \nu \leqslant l \leqslant k ; \quad \mathbf{z}_{\nu-1} \notin \pi, \quad \mathbf{z}_{k+1} \notin \pi ;
$$

- векторы $\mathbf{z}_{\nu-1}, \mathbf{z}_{\nu}, \mathbf{z}_{k}, \mathbf{z}_{k+1}$ линейно независимы.

Итак

$$
\begin{aligned}
1 & \leqslant\left|\operatorname{det}\left(\begin{array}{cccc}
y_{1, \nu-1} & y_{2, \nu-1} & y_{3, \nu-1} & x_{\nu-1} \\
y_{1, \nu} & y_{2, \nu} & y_{3, \nu} & x_{\nu} \\
y_{1, k} & y_{2, k} & y_{3, k} & x_{k} \\
y_{1, k+1} & y_{2, k+1} & y_{3, k+1} & x_{k+1}
\end{array}\right)\right| \\
& \leqslant 24 \zeta_{\nu-1} \zeta_{\nu} \zeta_{k} M_{k+1} \leqslant 24 \psi\left(M_{\nu}\right) \psi\left(M_{\nu+1}\right) \psi\left(M_{k+1}\right) M_{k+1} \\
& <24 \psi\left(M_{\nu}\right)\left(\psi\left(M_{\nu+1}\right)\right)^{2} M_{k+1} .
\end{aligned}
$$

Далее надо рассмотреть три случая.

$1^{\circ}$. Для некоторого $\gamma>1$ имеется бесконечно много пар $(\nu, k)$ рассматриваемого вида таких, что

$$
M_{k+1} \leqslant M_{\nu+1}^{\gamma}
$$

Из неравенства (61) получаем

$$
\frac{1}{24 \psi\left(M_{\nu}\right)} \leqslant M_{\nu+1}^{\gamma} \psi\left(M_{\nu+1}\right) \psi\left(M_{\nu+1}^{\gamma}\right) .
$$


Дополнительно предполагая возрастание функции $t \mapsto t^{\gamma} \psi(t) \psi\left(t^{\gamma}\right)$ и обозначая обратную к ней через $\rho(t)$, находим

$$
\zeta_{\nu} \leqslant \psi\left(M_{\nu+1}\right) \leqslant \psi\left(\rho\left(\frac{1}{24 \psi\left(M_{\nu}\right)}\right)\right) .
$$

$2^{\circ}$. Для некоторого $\delta \geqslant 1$ имеется бесконечно много рассматриваемых пар индексов $(\nu, k)$ таких, что

$$
M_{k+1} \geqslant M_{k}^{\delta}
$$

Тогда сразу получаем

$$
\zeta_{k} \leqslant \psi\left(M_{k+1}\right) \leqslant \psi\left(M_{k}^{\delta}\right) .
$$

$3^{\circ}$. Для бесконечного количества рассматриваемых пар индексов $(\nu, k)$ выполнено

$$
M_{\nu+1}^{\gamma} \leqslant M_{k+1} \leqslant M_{k}^{\delta}
$$

Тогда для двумерной решетки $\Lambda=\mathbb{Z}^{4} \cap \pi$ при $\nu \leqslant l \leqslant k-1$

$$
\zeta_{l} M_{l+1} \asymp_{\Theta} \operatorname{det}_{2} \Lambda \text {. }
$$

Отсюда получаем неравенства

$$
\zeta_{k-1} \ll_{\Theta} \frac{M_{\nu+1} \psi\left(M_{\nu+1}\right)}{M_{k}} \ll_{\Theta} M_{k}^{\delta / \gamma-1} \psi\left(M_{k}^{\delta / \gamma}\right) \leqslant M_{k-1}^{\delta / \gamma-1} \psi\left(M_{k-1}^{\delta / \gamma}\right)
$$

(конечно, надо дополнительно предполагать монотонное убывание функции $\left.t^{\delta / \gamma-1} \psi\left(t^{\delta / \gamma}\right)\right)$.

Для завершения доказательства надо рассмотреть малое $\varepsilon>0$ и положить $\psi(t)=t^{-\alpha+\varepsilon}$. В свете соотношения (59) неравенство (60) вытекает из (62)-(64).

5.3. Случай $m=2$. Сформулируем теорему В. Ярника о диофантовом типе из работы [13], аналогичную теореме 19.

ТеОрема 21. Пусть $m=2, n \geqslant 1$ и невырожденная матрица

$$
\Theta=\left(\begin{array}{cc}
\theta_{1}^{1} & \theta_{1}^{2} \\
\cdots \cdots & \ldots \\
\theta_{n}^{1} & \theta_{n}^{2}
\end{array}\right)
$$

является $\psi$-сингулярной с некоторой монотонно убывающей функцией $\psi(t)=$ $o\left(t^{-1}\right), t \rightarrow+\infty$. Положим

$$
\varphi_{2}^{[\psi]}(t)=\psi\left(\frac{1}{6 t \psi(t)}\right)
$$

Тогда найдется бесконечно много челочисленных векторов $\mathbf{x}=\left(x_{1}, x_{2}\right)$ таких, чmo

$$
\max _{1 \leqslant j \leqslant n}\left\|\theta_{j}^{1} x_{1}+\theta_{j}^{2} x_{2}\right\| \leqslant \varphi_{2}^{[\psi]}\left(\max _{i=1,2}\left|x_{i}\right|\right)
$$


Отметим, что в условиях теоремы для правильной матрицы $\Theta$ выполнено $R(\Theta) \geqslant 3$. При $n=1$ это следует из утверждения (iii) теоремы 7 . При $n \geqslant 2$ это вытекает из $\psi$-сингулярности и из теоремы 8 . Теперь для доказательства надо установить аналог следствия 4 п. 4.1 (с. 69). Для некоторого индекса $k$ и бесконечно многих значений $\nu$ составленный из координат векторов наилучших приближений определитель

$$
\operatorname{det}\left(\begin{array}{ccc}
x_{1, \nu} & x_{2, \nu} & y_{k, \nu} \\
x_{1, \nu+1} & x_{2, \nu+1} & y_{k, \nu+1} \\
x_{1, \nu+2} & x_{2, \nu+2} & y_{k, \nu+2}
\end{array}\right)
$$

не обращается в нуль (на самом деле это место требует более подробного объяснения). Затем для такого значения $\nu$ надо провести оценку, аналогичную (51):

$$
1 \leqslant 6 M_{\nu+2} M_{\nu+1} \zeta_{\nu} \leqslant 6 M_{\nu+2} M_{\nu+1} \psi\left(M_{\nu+1}\right)
$$

И наконец, надо воспользоваться монотонностью функции $\psi$.

Мы не останавливаемся здесь на доказательстве подробно (например, следует разобраться с ситуацией, когда матрица $\Theta$ не является правильной). Все детали имеются в оригинальной работе В. Ярника.

Из теоремы 21 В. Ярник выводит следующее утверждение.

СлеДСтвиЕ. Пусть $m=2, n \geqslant 1$ и матрица $\Theta$ невырожденная. Тогда для величин $\alpha(\Theta), \beta(\Theta)$, определяемых как супремум тех $\gamma$, для которых выполнено (56) и (57) соответственно, имеет место соотношение

$$
\beta(\Theta) \geqslant \alpha(\Theta)(\alpha(\Theta)-1) .
$$

5.4. Случай $m=n=2$. Для $\alpha \geqslant 1$ пусть

$$
g_{3}(\alpha)=\frac{1-\alpha+\sqrt{(1-\alpha)^{2}+4 \alpha\left(2 \alpha^{2}-2 \alpha+1\right)}}{2 \alpha}
$$

есть решение уравнения

$$
\alpha x^{2}+(\alpha-1) x-\left(2 \alpha^{2}-2 \alpha+1\right)=0 .
$$

Заметим, что $g_{3}(1)=1$ и при $\alpha>1$ выполнено $g_{3}(\alpha)>1$. Более того, при $1 \leqslant \alpha<((1+\sqrt{5}) / 2)^{2}$ выполнено

$$
g_{3}(\alpha)>\max (1, \alpha-1) .
$$

ТЕОРема 22. Пусть числа $\theta_{j}^{i}, i, j=1,2$, линейно независимы вместе с единицей над $\mathbb{Z}$. Рассмотрим матрииу

$$
\Theta=\left(\begin{array}{ll}
\theta_{1}^{1} & \theta_{1}^{2} \\
\theta_{2}^{1} & \theta_{2}^{2}
\end{array}\right)
$$

Тогда

$$
\beta(\Theta) \geqslant \alpha(\Theta) g_{3}(\alpha(\Theta))
$$

Теорема 22 сильнее теоремы 21 при $\alpha(\Theta) \in\left(1,((1+\sqrt{5}) / 2)^{2}\right)$. 
Доказательство. Если $R(\Theta)=2$, то из теоремы 8 получаем $\alpha(\Theta)=1$, и доказывать нечего. Согласно следствию 4 из п. 2.1 (с. 57$)$, равенство $R(\Theta)=3$ выполняться не может. Итак, $R(\Theta)=4$. Тогда для бесконечной последовательности пар индексов $\nu<k$ при $\nu \rightarrow+\infty$ имеют место следующие три утверждения:

- обе тройки

$$
\left(\mathbf{z}_{\nu-1}, \mathbf{z}_{\nu}, \mathbf{z}_{\nu+1}\right), \quad\left(\mathbf{z}_{k-1}, \mathbf{z}_{k}, \mathbf{z}_{k+1}\right),
$$

состоят из линейно независимых векторов;

- имеется двумерное подпространство $\pi$ такое, что

$$
\mathbf{z}_{l} \in \pi, \quad \nu \leqslant l \leqslant k ; \quad \mathbf{z}_{\nu-1} \notin \pi, \quad \mathbf{z}_{k+1} \notin \pi ;
$$

- векторы $\mathbf{z}_{\nu-1}, \mathbf{z}_{\nu}, \mathbf{z}_{k}, \mathbf{z}_{k+1}$ линейно независимы.

Теперь

$$
1 \leqslant\left|\operatorname{det}\left(\begin{array}{cccc}
x_{1, \nu-1} & x_{2, \nu-1} & y_{1, \nu-1} & y_{2, \nu-1} \\
x_{1, \nu} & x_{2, \nu} & y_{1, \nu} & y_{2, \nu} \\
x_{1, k} & x_{2, k} & y_{1, k} & x_{2, k} \\
x_{1, k+1} & x_{2, k+1} & y_{1, k+1} & y_{2, k+1}
\end{array}\right)\right| \leqslant 24 \zeta_{\nu-1} \zeta_{\nu} M_{k} M_{k+1} .
$$

Далее предполагаем, что $\psi(t)$ монотонно убывает. Пусть снова $\psi_{\Theta}(t) \leqslant \psi(t)$. Тогда

$$
1 \leqslant 24 M_{k+1} M_{k} \psi\left(M_{\nu+1}\right) \psi\left(M_{\nu}\right) .
$$

Рассмотрим два случая.

$1^{\circ}$. Для некоторого $\gamma>1$ существует бесконечно много рассматриваемых пар $(\nu, k) \mathrm{c}$

$$
M_{k+1} \geqslant M_{k}^{\gamma}
$$

Тогда немедленно получаем

$$
\zeta_{k} \leqslant \psi\left(M_{k+1}\right) \leqslant \psi\left(M_{k}^{\gamma}\right)
$$

$2^{\circ}$. Для бесконечного количества рассматриваемых пар $(\nu, k)$ выполнено

$$
M_{k+1} \leqslant M_{k}^{\gamma}
$$

Тогда из (67) следует, что

$$
M_{k} \geqslant\left(\psi\left(M_{\nu}\right)\right)^{-2 /(1+\gamma)}
$$

Рассмотрим двумерную подрешетку $\Lambda=\pi \cap \mathbb{Z}^{4}$ с определителем $\operatorname{det} \Lambda$. Расстояние от точки $\mathbf{z} \in \pi$ до двумерного подпространства

$$
\mathscr{L}=\left\{\mathbf{z}=\left(x_{1}, x_{2}, y_{1}, y_{2}\right): \theta_{1}^{1} x_{1}+\theta_{1}^{2} x_{2}+y_{1}=\theta_{2}^{1} x_{1}+\theta_{2}^{2} x_{2}+y_{2}=0\right\}
$$

пропорционально расстоянию от $\mathbf{z}$ до одномерного подпространства $\mathscr{L} \cap \pi$. Пусть $\delta$ - коэффициент этой пропорциональности. Параллелепипед

$$
\left\{\mathbf{z}=\left(x_{1}, x_{2}, y_{1}, y_{2}\right):|x|<M_{l+1}, \max _{1 \leqslant j \leqslant 3}\left|\theta_{j} x-y_{j}\right|<\zeta_{l}\right\}
$$


не содержит внутри себя ненулевых целых точек. Значит,

$$
\gamma_{1}(\Theta) \delta \operatorname{det} \Lambda \leqslant \zeta_{l} M_{l+1} \leqslant \gamma_{2}(\Theta) \delta \operatorname{det} \Lambda, \quad \nu \leqslant l \leqslant k-1,
$$

с некоторыми постоянными $\gamma_{i}(\Theta), i=1,2$. Из (69) и (70) выводим, что

$$
\zeta_{\nu} \ll_{\Theta} \frac{\psi\left(M_{k}\right) M_{k}}{M_{\nu+1}} \ll_{\Theta} M_{\nu}^{-1}\left(\psi\left(M_{\nu}\right)\right)^{-2 /(1+\gamma)} \psi\left(\left(\psi\left(M_{\nu}\right)\right)^{-2 /(1+\gamma)}\right) .
$$

Далее рассматриваем функцию $\psi(t)=t^{-\alpha(\Theta)+\varepsilon}$ с малым положительным $\varepsilon$. Поскольку величина $\gamma=g_{3}(\alpha(\Theta))$ удовлетворяет (66), то получаем утверждение теоремы 22 .

5.5. Случай $m>2$. В случае $m>2$ В. Ярник в [13] использует более громоздкие рассуждения. Поэтому он формулирует и доказывает не общий результат с произвольной функцией $\psi$, а только лишь утверждение про диофантовы показатели.

ТеОРема 23. Пусть $m \geqslant 3$ и про невырожденную матрииу $\Theta$ известно, что

$$
\alpha(\Theta)>\left(5 m^{2}\right)^{m-1} .
$$

Тогда

$$
\beta(\Theta) \geqslant(\alpha(\Theta))^{m /(m-1)}-3 \alpha(\Theta) .
$$

Доказательство этой теоремы мы здесь не приводим. Оно основывается на изящном построении последовательности независимых векторов приближений. У автора нет сомнений в том, что результат теоремы 23 не является оптимальным и может быть улучшен. В следующем пункте мы как раз и приводим подобного рода улучшение (в частном случае).

5.6. Случай $m=3, n=1$. Для $\alpha \geqslant 3$ определим функции

$$
g_{2}(\alpha)=\sqrt{\alpha+\frac{1}{\alpha^{2}}-\frac{7}{4}}+\frac{1}{\alpha}-\frac{1}{2}, \quad h(\alpha)=\alpha-g(\alpha)-1 .
$$

Заметим, что функции $g_{2}(\alpha)$ и $h(\alpha)$ монотонно возрастают к бесконечности при $\alpha \rightarrow+\infty$ и

$$
g_{2}(3)=h(3)=1, \quad g_{2}(\alpha) \leqslant \alpha-2 .
$$

Теорема 24. Рассмотрим матрицу-строку $\Theta=\left(\theta^{1}, \theta^{2}, \theta^{3}\right)$, состоящую из линейно независимых вместе $с$ единицей над $\mathbb{Z}$ чисел. Тогда показатели $\alpha(\Theta)$ и $\beta(\Theta)$ связаны соотношением

$$
\beta(\Theta) \geqslant \alpha(\Theta) g_{2}(\alpha(\Theta))
$$

ДокАЗАТЕЛьство. Мы будем рассматривать последовательности векторов наилучших приближений $\mathbf{z}_{\nu}$.

Сначала рассмотрим случай, когда $R(\Theta)=3$. Тогда все векторы $\mathbf{z}_{\nu}$, начиная с какого-то номера, лежат в некотором трехмерном вполне рациональном подпространстве $\pi$, и мы фактически имеем дело с последовательностью наилучших приближений точками трехмерной решетки $\mathbb{Z}^{4} \cap \pi$ двумерного подпространства $\pi \cap \mathscr{L}(\Theta)$ (напомним, что определение подпространства $\mathscr{L}(\Theta)$ мы 
давали в п. 2.1). Тогда применима теорема 21, и ее следствие дает оценку (65), что сильнее, чем (72).

Теперь рассмотрим случай, когда $R(\Theta)=4$. В этом случае найдется бесконечно много пар индексов $\nu<k(\nu \rightarrow+\infty)$ таких, что:

- тройки

$$
\mathbf{z}_{\nu-1}, \mathbf{z}_{\nu}, \mathbf{z}_{\nu+1} ; \quad \mathbf{z}_{k-1}, \mathbf{z}_{k}, \mathbf{z}_{k+1}
$$

состоят из линейно независимых векторов каждая;

- имеется некоторое двумерное вполне рациональное подпространство $\pi$ такое, что

$$
\mathbf{z}_{l} \in \pi, \quad \nu \leqslant l \leqslant k ; \quad \mathbf{z}_{\nu-1} \notin \pi, \quad \mathbf{z}_{k+1} \notin \pi ;
$$

- четыре вектора $\mathbf{z}_{\nu-1}, \mathbf{z}_{\nu}, \mathbf{z}_{\nu+1}, \mathbf{z}_{k+1}$ линейно независимы.

Тогда, предполагая, что набор $\Theta$ является $\psi$-сингулярным (естественно, считаем, что функции $\psi(t)$ и $t \psi(t)$ монотонны), получаем

$$
\begin{aligned}
1 & \leqslant\left|\operatorname{det}\left(\begin{array}{cccc}
x_{1, \nu-1} & x_{2, \nu-1} & x_{3, \nu-1} & y_{\nu-1} \\
x_{1, \nu} & x_{2, \nu} & x_{3, \nu} & y_{\nu} \\
x_{1, \nu+1} & x_{2, \nu+1} & x_{3, \nu+1} & y_{\nu+1} \\
x_{1, k+1} & x_{2, k+1} & x_{3, k+1} & y_{k+1}
\end{array}\right)\right| \\
& \leqslant 24 \zeta_{\nu-1} M_{\nu} M_{\nu+1} M_{k+1} \leqslant 24 \psi\left(M_{\nu}\right) M_{\nu} M_{\nu+1} M_{k+1} .
\end{aligned}
$$

Далее рассматриваем три случая.

$1^{\circ}$. Если для бесконечного количества рассматриваемых пар индексов $(\nu, k)$ выполнено

$$
M_{k+1} \leqslant M_{\nu}^{h(\alpha(\Theta))},
$$

то для таких пар индексов из (73) получаем

$$
M_{\nu+1} \geqslant \frac{1}{24 \psi\left(M_{\nu}\right) M_{\nu}^{1+h(\alpha(\Theta))}}, \quad \zeta_{\nu} \leqslant \psi\left(\frac{1}{24 \psi\left(M_{\nu}\right) M_{\nu}^{1+h(\alpha(\Theta))}}\right) .
$$

Из последнего неравенства требуемое соотношение (72) сразу вытекает.

$2^{\circ}$. Пусть для бесконечного количества рассматриваемых пар индексов $(\nu, k)$ выполнено

$$
M_{k+1} \geqslant M_{k}^{g_{2}(\alpha(\Theta))} .
$$

В этом случае сразу получаем

$$
\zeta_{k} \leqslant \psi\left(M_{k+1}\right) \leqslant \psi\left(M_{k}^{g_{2}(\alpha(\Theta))}\right),
$$

откуда следует соотношение (72).

$3^{\circ}$. Пусть для бесконечного количества рассматриваемых пар индексов $(\nu, k)$ выполнено

$$
M_{\nu}^{h(\alpha(\Theta))} \leqslant M_{k+1} \leqslant M_{k}^{g_{2}(\alpha(\Theta))} .
$$

В этом случае надо более детально исследовать, что происходит в подпространстве $\pi$. Рассмотрим проекцию подпространства $\pi$ на подпространство $\mathscr{L}(\Theta)$. Это есть некоторое двумерное линейное подпространство $\pi^{*}$, пересекающееся с подпространством $\pi$ по некоторой прямой $\ell=\pi \cap \pi^{*}$. Для точки $\mathbf{z} \in \pi$ 
расстояния от $\mathbf{z}$ до подпространства $\mathscr{L}(\Theta)$ и от $\mathbf{z}$ до подпространства $\ell$ пропорциональны. Обозначим через $\delta$ коэффициент этой пропорциональности. Так как векторы $\mathbf{z}_{l}$ являются векторами наилучших приближений, то как векторы двумерной решетки $\Lambda=\mathbb{Z}^{4} \cap \pi$ они автоматически оказываются наилучшими приближениями точками решетки $\Lambda$ прямой $\ell$ в индуцированной норме. Пусть $\operatorname{det} \Lambda$ есть фундаментальный объем решетки $\Lambda$. Ясно, что для некоторых положительных постоянных $\gamma_{i}(\Theta), i=1,2$, выполнено

$$
\gamma_{1}(\Theta) \delta \operatorname{det} \Lambda \leqslant \zeta_{l} M_{l+1} \leqslant \gamma_{2}(\Theta) \delta \operatorname{det} \Lambda, \quad \nu \leqslant l \leqslant k-1 .
$$

В частности,

$$
\zeta_{\nu} M_{\nu+1} \leqslant \frac{\gamma_{2}(\Theta)}{\gamma_{1}(\Theta)} \zeta_{k-1} M_{k}
$$

Учтем, что $\zeta_{k-1} \leqslant \psi\left(M_{k}\right)$. Из условия рассматриваемого случая получаем

$$
\zeta_{\nu} \leqslant \frac{\gamma_{2}(\Theta)}{\gamma_{1}(\Theta)} \frac{\psi\left(M_{k}\right) M_{k}}{M_{\nu+1}} \leqslant \frac{\gamma_{2}(\Theta)}{\gamma_{1}(\Theta)} \psi\left(M_{\nu}^{h(\alpha(\Theta)) / g_{2}(\alpha(\Theta))}\right) M_{\nu}^{h(\alpha(\Theta)) / g_{2}(\alpha(\Theta))-1} .
$$

Поскольку

$$
\alpha\left(g_{2}(\alpha)\right)^{2}+(\alpha-2) g_{2}(\alpha)-(\alpha-1)^{2}=0,
$$

то снова получаем (72).

Теорема доказана.

\section{6. Неоднородные приближения}

6.1. Одномерные задачи. Для вещественных $\theta$ и $\alpha$ рассмотрим величину

$$
\lambda(\theta, \alpha)=\liminf _{x \rightarrow \infty}|x| \cdot\|x \theta-\alpha\|
$$

(здесь подразумевается, что $x$ принимает целые значения). Согласно классическому результату Г. Минковского (см. [23; гл. III, теорема II]), для любого $\theta$ и $\alpha$, не представимого в виде $a \theta+b$ с целыми $a, b$, выполняется неравенство

$$
\lambda(\theta, \alpha) \leqslant \frac{1}{4}
$$

Положим

$$
\lambda(\theta)=\lambda(\theta, 0) \quad \text { и } \quad \mu(\theta)=\sup _{\alpha} \lambda(\theta, \alpha),
$$

где супремум берется по всем $\alpha$, не представимым в виде $a \theta+b$ с целыми $a, b$.

А. Я. Хинчин в работе [5] доказал неравенство

$$
\mu(\theta) \leqslant \frac{\sqrt{1-4 \lambda^{2}(\theta)}}{4},
$$

которое для некоторых $\theta$ является точным. Например, если $\theta$ эквивалентно числу $[0 ; k, k, k, \ldots]$, задаваемому периодической непрерывной дробью, и неполное частное $k$ либо равно единице, либо четно, то в неравенстве (74) имеет место знак равенства. Из (74) также следует, что равенство $\mu(\theta)=1 / 4$ возможно 
только для таких $\theta$, разложение которых в непрерывную дробь имеет сколь угодно большие неполные частные.

В работе А. Я. Хинчина [5] имеется ряд других результатов. Изучению множеств значений функции $\lambda(\theta, \alpha)$ и ей подобных, например, "односторонней" функции

$$
k(\theta, \alpha)=\liminf _{x \rightarrow+\infty} x\|x \theta-\alpha\|,
$$

посвящены, например, работы Дж. В. С. Касселса [45], Е. Барнса [46], Т. Кузика и А. Поллингтона [47].

Сейчас мы сформулируем простой одномерный (но тем не менее весьма нетривиальный) результат, который содержится все в той же работе А. Я. Хинчина [1] (Satz 4).

ТЕОРема 25. Существует абсолютная константа $\gamma$ со следующим свойством: для каждого вещественного $\theta$ можно найти вещественное а такое, что для всех натуральных $x$ имеет место неравенство

$$
\|x \theta-\alpha\| \geqslant \frac{\gamma}{x} .
$$

Отметим, что в книге Касселса [23] результат теоремы 25 имеется с постоянной $\gamma=1 / 51$ (см. [23; гл. V, теорему XI]; там же имеется непонятное замечание к главе $\mathrm{V}$, в котором речь идет об оценке точного значения постоянной $\gamma$; по-видимому, самый лучший результат принадлежит Г. Годвину [48]).

В дальнейшем мы обсудим и многомерные обобщения теоремы 25, и моменты ее доказательства, поскольку доказательство теоремы 25 послужило отправной точкой для всех последующих многомерных результатов, в том числе для теорем В. Ярника, о которых пойдет речь в следующем пункте.

6.2. Многомерные теоремы. При доказательстве теоремы 25 обнаружилась связь между однородными и неоднородными задачами в теории линейных диофантовых приближений. Эта связь получила дальнейшее развитие; причем оказалось, что характеристики сингулярности соответствующих однородных систем играют решающую роль. А. Я. Хинчин неоднократно возвращался к исследованию неоднородных линейных приближений. В работе 1936 г. [4] он доказал основополагающий многомерный результат (точнее, утверждение было сформулировано для произвольного $m$, а доказано при $m=2$ ), который мы здесь приводим.

Теорема 26 (А.Я. Хинчин [4]). Следующие два условия (i) и (ii) эквивалентны:

(i) для набора вещественных чисел $\theta_{1}, \ldots, \theta_{m}$ имеет место неравенство

$$
\limsup _{t \rightarrow+\infty} t^{m} \min _{\mathbf{x} \in \mathbb{Z}^{m}: 0<M(\mathbf{x}) \leqslant t}\left\|\sum_{1 \leqslant i \leqslant m} \theta_{i} x_{i}\right\|>0 ;
$$

(ii) набор вещественных чисел $\theta_{1}, \ldots, \theta_{m}$ таков, что для любого вещественного числа а выполнено

$$
\liminf _{t \rightarrow+\infty} t^{m} \min _{\mathbf{x} \in \mathbb{Z}^{m}: M(\mathbf{x}) \leqslant t}\left\|\sum_{1 \leqslant i \leqslant m} \theta_{i} x_{i}-\alpha\right\|<+\infty .
$$


Очевидно, что условие (i) эквивалентно регулярности набора $\Theta$ (1) с заданным значением $m$ и с $n=1$.

Хочется отметить, что доказательства всех дальнейших результатов, обсуждаемых в настоящем пункте, развивают идеи работ А. Я. Хинчина [1], [4].

В 1948 г. в работе [7] А.Я. Хинчин доказал общее утверждение, формулировку которого мы тоже хотим здесь привести.

Система $\Theta$ называется системой Чебышёва, если для любого набора вещественных чисел $\alpha=\left(\alpha_{1}, \ldots, \alpha_{n}\right) \in \mathbb{R}^{n}$ найдется постоянная $\Gamma=\Gamma(\alpha)$ такая, что система диофантовых неравенств

$$
\max _{1 \leqslant j \leqslant n}\left\|L_{j}(\mathbf{x})-\alpha_{j}\right\| \leqslant \Gamma\left(\max _{1 \leqslant i \leqslant m}\left|x_{i}\right|\right)^{m / n}
$$

имеет решения со сколь угодно большим значением величины $\max _{1 \leqslant i \leqslant m}\left|x_{i}\right|$.

Основной результат работы [7] состоит в следующем.

Теорема 27. Система $\Theta$ является регулярной тогда и только тогда, когда она система Чебышёва.

Таким образом, теорема 26, сформулированная выше, является частным случаем теоремы $27(n=1)$.

Доказательство этой теоремы, помимо оригинальной работы А.Я. Хинчина [1], изложено, например, в [23; гл. Х].

Следует отметить, что несколько ранее (в работах [10] и [11] соответственно 1939 и 1941 гг.; см. также [12]) В. Ярник получил более общие утверждения, формулировки которых мы приводим ниже.

Наряду с “однородной” функцией Ярника (20) удобно использовать “неоднородную" функцию

$$
\psi_{\Theta, \alpha}(t)=\min _{\mathbf{x} \in \mathbb{R}^{m}: M(\mathbf{x}) \leqslant t} \max _{1 \leqslant j \leqslant n}\left\|L_{j}(\mathbf{x})-\alpha_{j}\right\|, \quad \alpha=\left(\alpha_{1}, \ldots, \alpha_{n}\right) \in \mathbb{R}^{n},
$$

и функцию

$$
\Psi_{\Theta}^{[\mathrm{inhom}]}=\sup _{\alpha \in[0,1]^{n}} \psi_{\Theta, \alpha}(t)
$$

В определении "неоднородной" функции Ярника можно брать минимум по всем ненулевым векторам х. Результат от этого не изменится.

Ясно, что функция Ярника (20) удовлетворяет равенству

$$
\psi_{\Theta}(t)=\psi_{\Theta, 0}(t)
$$

Также наряду с системой чисел $\Theta$ будем рассматривать транспонированную систему ${ }^{t} \Theta$. Для функции Ярника $\psi_{t_{\Theta}}(t)$, очевидно, имеем равенство

$$
\psi_{t \Theta}(t)=\min _{\mathbf{x} \in \mathbb{R}^{n}: M(x)<t} \max _{1 \leqslant i \leqslant m}\left\|L_{i}^{*}(\mathbf{x})\right\|,
$$

где

$$
L_{i}^{*}(\mathbf{x})=\sum_{j=1}^{n} \theta_{j}^{i} x_{j}
$$


Всюду ниже в этом пункте предполагаем, что функция $\psi(t)$ строго монотонно убывает к нулю. Обозначим через $\rho(t)$ функцию, обратную к функции $1 / \psi(t)$.

Сначала мы сформулируем более простой результат, который содержится в работе [10] (теорему 1 из [10]).

Теорема 28 (В. Ярник [10]). Пусть функиия $\psi(t)$ такова, что при некотором положительном $\eta$ бункция $1 /\left(t^{\eta} \psi(t)\right)$ монотонно стремится $\kappa$ бесконечности при $t \rightarrow+\infty$. Предположим, что при всех достаточно больших значениях $t$ имеет место неравенство

$$
\psi_{t_{\Theta}(t)}>\psi(t)
$$

Тогда для всех достаточно больших значений $t$ выполнено

$$
\Psi_{\Theta}^{[\text {inhom }]} \leqslant \frac{((m+n) !(m+n))^{(\eta+1) / \eta}}{\rho(t)} .
$$

При $\psi_{t} \Theta=c t^{n / m}$, где $c$ - положительная постоянная, из теоремы 28 получаем следующее утверждение.

СлЕДСТвИЕ. Если транспонированная система ${ }^{t} \Theta$ является регулярной, то система $\Theta$ является системой Чебишёва.

Следующая теорема фактически есть результат работы В. Ярника [11] (теорема 7 из [11], см. также [12], отличается от формулируемой ниже теоремы 29 только тем, что в ней не выписаны в явном виде константы).

ТеОрема 29. Пусть при всех достаточно больиих $t$ имеет место неравенство

$$
\psi_{t_{\Theta}}(t) \leqslant \psi(t)
$$

Тогда найдется набор вещественных чисел $\left(\alpha_{1}, \ldots, \alpha_{n}\right)$, для которого при всех достаточно больиих $t$ выполнено

$$
\psi_{\Theta, \alpha}(t) \geqslant \frac{1}{24 n^{3 / 2} \rho(8 m t)} .
$$

В рассматриваемом вопросе это утверждение представляет основную сложность. Оно восходит к теореме А. Я. Хинчина 25, которая была доказана в [1]. Доказательство именно этой теоремы мы прокомментируем в следующем пункте. Здесь же мы отметим два важных следствия.

Полагая $\psi_{t_{\Theta}}=o\left(t^{-n / m}\right), t \rightarrow+\infty$, получаем следующее утверждение.

СлЕДСтвиЕ. Если транспонированная система ${ }^{t} \Theta$ сингулярна, то система $\Theta$ не есть система Чебышёва.

Это следствие вместе со следствием теоремы 28 приводит к утверждению, которое естественно считать доказанным В. Ярником.

Теорема 30. Транспонированная система ${ }^{t} \Theta$ является регулярной тогда и только тогда, когда она является системой Чебышёва. 
Чтобы убедиться в эквивалентности теоремы 27, доказанной А.Я. Хинчиным, и теоремы 30, доказанной В. Ярником, надо вспомнить следующую теорему переноса из работы А. Я. Хинчина 1948 г, [6] (она излагается также в книге [23], гл. V, теорема XII).

ТеОрема 31. Система $\Theta$ сингулярна тогда и толъко тогда, когда сингулярна транспонированная система ${ }^{t} \Theta$.

Но в работе В. Ярника 1941 г. [11] имеется следующее утверждение.

Теорема 32. Если с некоторой положительной постоянной $\gamma_{1}$ при всех достаточно больших значениях $t$ выполнено неравенство

$$
\psi_{\Theta}(t) \geqslant \gamma_{1} t^{-m / n}
$$

то с некоторой положительной постоянной $\gamma_{2}$ при всех достаточно больших значениях $t$ выполняется

$$
\psi_{t_{\Theta}}(t) \geqslant \gamma_{2} t^{-n / m} .
$$

Теоремы 31 и 32 очевидным образом эквивалентны!

О других теоремах переноса пойдет речь в разделе 8.

Совокупное доказательство теорем 28 и 29 В. Ярника мало чем отличается от доказательства теоремы 31, данного А. Я. Хинчиным. Хинчин доказывает сначала, что если система $\Theta$ является регулярной, то она есть система Чебышёва. Для этого достаточно применить теорему Минковского к системе $m+n$ линейных форм от $m+n+1$ переменных $x_{1}, \ldots, x_{n}, y_{1}, \ldots, y_{m}, u$ вида

$$
L_{j}(\mathbf{x})-y_{j}-\xi_{j} u, \quad j=1, \ldots, n, \quad x_{j}, \quad j=1, \ldots, m
$$

(здесь $\xi_{j}$ суть новые вещественные параметры). Затем, с помощью применения конструктивных соображений (о них пойдет речь в п. 6.3) и соображений переноса, он доказывает обратное утверждение. Ярник же соображения переноса применяет в теореме 28, а конструктивной теоремой является теорема 29.

Обсудим еще одно следствие теоремы 29. Согласно теореме Минковского о выпуклом теле, упоминавшейся в самом начале раздела 1, если положить $\psi(t)=t^{-n / m}$, то условие выполнения неравенства (77) из теоремы В. Ярника 29 становится пустым условием. Теперь в качестве следствия мы получаем утверждение, имеющееся в книге Дж. В.С. Касселса [23; гл. V, теорема X].

ТЕОРема 33. Для произвольных натуральных $n$ и $m$ существует положительная постоянная $\Gamma_{m, n}$, обладающая следующим свойством. Для любой системы $\Theta$ найдется неоднородность $\left(\alpha_{1}, \ldots, \alpha_{n}\right)$ такая, что

$$
\inf _{\mathbf{x} \in \mathbb{Z}^{m} \backslash\{\mathbf{0}\}}\left(\max _{1 \leqslant j \leqslant n}\left\|L_{j}(\mathbf{x})-\alpha_{j}\right\|\right)^{n}\left(\max _{1 \leqslant i \leqslant m}\left|x_{i}\right|\right)^{m}>\Gamma_{m, n} .
$$

Эта теорема является непосредственным обобщением теоремы 25, доказанной А.Я. Хинчиным в [1]. С ней связана интересная история, о которой мы расскажем в п. 6.4.

В заключение настоящего пункта отметим, что в замечательной работе В. Ярника [11] имеется еще несколько теорем, о которых мы здесь не упомянули (в частности, там имеется результат метрического характера). 
6.3. О доказательстве теоремы 29. Доказательство теоремы 33, имеющееся в книге [23], непосредственно переделывается в доказательство теоремы 29. Оригинальные рассуждения В. Ярника несколько более громоздки. Мы приведем здесь схему доказательства теоремы 29, следуя книге [23]. Приводя схему доказательства, мы ограничимся случаем, когда все числа $\theta_{j}^{i}$ линейно независимы вместе с единицей над $\mathbb{Z}$ (исключительно для удобства изложения, чтобы избежать разбора нескольких случаев).

1. Последовательность наилучших приближений

$$
\mathbf{w}_{\nu}=\left(u_{1, \nu}, \ldots, u_{n, \nu}, v_{1, \nu}, \ldots, v_{m, \nu}\right)
$$

для транспонированной системы ${ }^{t} \Theta$ надо “проредить" таким образом, чтобы получилась последовательность $\mathbf{w}_{\nu_{k}}$ такая, что

$$
\begin{gathered}
\max _{1 \leqslant i \leqslant m}\left\|L_{i}^{*}\left(\mathbf{u}_{\nu_{k}}\right)\right\|=\psi_{t}\left(\frac{M\left(\mathbf{u}_{\nu_{k+1}}\right)}{3 \sqrt{n}}\right), \\
M\left(\mathbf{u}_{\nu+1}\right) \geqslant 3 \sqrt{n} M\left(\mathbf{u}_{\nu}\right), \quad M\left(\mathbf{u}_{\nu}\right)=\max _{1 \leqslant j \leqslant n}\left|u_{j, \nu+1}\right|
\end{gathered}
$$

(см. лемму $4 \S 6$ главы V [23], или рассуждения из $\S 2$, п. 2 из [7], или конструкцию леммы 1 из [11]).

2. Необходимо следующее утверждение о диофантовых приближениях с лакунарной последовательностью векторов. Приводимая ниже формулировка взята из [23] (лемма 2 в $\S 6$ главы V). Она непосредственно обобщает лемму А. Я. Хинчина (Hilfssatz 3 из [1]). Этому утверждению и его развитию посвящен п. 13.1 добавления в настоящем обзоре.

Лемма 2. Для последовательности векторов $\mathbf{u}_{k}=\left(u_{1, k}, \ldots, u_{n, k}\right) \in \mathbb{R}^{n}$, удовлетворяющей неравенствам

$$
\max _{1 \leqslant j \leqslant n}\left|u_{j, k+1}\right| \geqslant 3 \sqrt{n} \max _{1 \leqslant j \leqslant n}\left|u_{j, k}\right|,
$$

найдутся вещественные числа $\alpha_{1}, \ldots, \alpha_{n}$ такие, что

$$
\left\|\sum_{1 \leqslant j \leqslant n} u_{j, k} \alpha_{j}\right\| \geqslant \frac{1}{4}, \quad k=1,2,3, \ldots .
$$

Наличие дополнительного (по сравнению с соответствующими утверждениями из [23]) множителя $\sqrt{n}$ связано с тем, что мы рассматриваем sup-норму, а не евклидову норму.

3. Далее доказывается, что числа $\alpha_{j}$ и есть те самые неоднородности, существование которых утверждается в теореме 29. Для этого используется тождество

$$
\begin{aligned}
\sum_{1 \leqslant j \leqslant n} u_{j} \alpha_{j} & =\sum_{1 \leqslant j \leqslant n} u_{j}\left(\alpha_{j}-L_{j}(\mathbf{x})\right)+\sum_{1 \leqslant j \leqslant n} u_{j} L_{j}(\mathbf{x}) \\
& =\sum_{1 \leqslant j \leqslant n} u_{j}\left(\alpha_{j}-L_{j}(\mathbf{x})\right)+\sum_{1 \leqslant i \leqslant m} x_{i} L_{i}^{*}(\mathbf{u}),
\end{aligned}
$$


где, естественно,

$$
\mathbf{x}=\left(x_{1}, \ldots, x_{m}\right) \in \mathbb{R}^{m}, \quad \mathbf{u}=\left(u_{1}, \ldots, u_{n}\right) \in \mathbb{R}^{n} .
$$

В этом тождестве (примененном для вектора $\mathbf{u}_{\nu_{k}}$, определенного в п. 1 доказательства (см. (79)), чисел $\alpha_{j}$ из леммы 2 и произвольного вектора $\mathbf{x} \in \mathbb{R}^{m}$ ) переходим к модулям и используем лемму 2 :

$$
\frac{1}{4} \leqslant n M\left(\mathbf{u}_{\nu_{k}}\right) \max _{1 \leqslant j \leqslant n}\left\|L_{j}(\mathbf{x})-\alpha_{j}\right\|+m \max _{1 \leqslant i \leqslant m}\left|x_{i}\right| \cdot \psi_{t_{\Theta}}\left(\frac{M\left(\mathbf{u}_{\nu_{k+1}}\right)}{3 \sqrt{n}}\right) .
$$

Теперь, если по заданному вектору х выбрать $k$ из условия

$$
\psi_{t_{\Theta}}\left(\frac{M\left(\mathbf{u}_{\nu_{k}}\right)}{3 \sqrt{n}}\right) \geqslant \frac{1}{8 m \max _{1 \leqslant i \leqslant m}\left|x_{i}\right|} \geqslant \psi_{t_{\Theta}}\left(\frac{M\left(\mathbf{u}_{\nu_{k+1}}\right)}{3 \sqrt{n}}\right),
$$

то получается

$$
\max _{1 \leqslant j \leqslant n}\left\|L_{j}(\mathbf{x})-\alpha_{j}\right\| \geqslant \frac{1}{8 n M\left(\mathbf{u}_{\nu_{k}}\right)} .
$$

Но из условия (77) теоремы и соотношения (80) находим

$$
\psi\left(\frac{M\left(\mathbf{u}_{\nu_{k}}\right)}{3 \sqrt{n}}\right) \geqslant \psi_{t_{\Theta}}\left(\frac{M\left(\mathbf{u}_{\nu_{k}}\right)}{3 \sqrt{n}}\right) \geqslant \frac{1}{8 m \max _{1 \leqslant i \leqslant m}\left|x_{i}\right|} .
$$

Отсюда, по определению функции $\rho(\cdot)$, получаем

$$
\rho\left(8 m \max _{1 \leqslant i \leqslant m}\left|x_{i}\right|\right) \geqslant \frac{M\left(\mathbf{u}_{\nu_{k}}\right)}{3 \sqrt{n}} .
$$

Подстановка последнего неравенства в неравенство (81) дает именно (78) с $t=$ $\max _{1 \leqslant i \leqslant m}\left|x_{i}\right|$.

6.4. О теореме 33. В последнее время к линейным неоднородным диофантовым приближениям вновь пробудился интерес. В частности, теорема 33 уточнялась несколькими авторами. Здесь мы изложим историю вопроса, а также схему доказательства наиболее сильного на настоящее время результата.

Следующую теорему доказал Д. Клейнбок [49].

Теорема 34. Через $\mathscr{B}$ обозначим множество вещественных матрии размера $(m+1) \times n$ вида

$$
\left(\theta_{j}^{i}, \eta_{j}\right), \quad 1 \leqslant i \leqslant m, \quad 1 \leqslant j \leqslant n,
$$

для которых выполняется неравенство

$$
\inf _{\mathbf{x} \in \mathbb{Z}^{m} \backslash\{\mathbf{0}\}}\left(\max _{1 \leqslant j \leqslant n}\left\|L_{j}(\mathbf{x})-\eta_{j}\right\|\right)^{n}\left(\max _{1 \leqslant i \leqslant m}\left|x_{i}\right|\right)^{m}>0 .
$$

Тогда $\mathscr{B}$ есть множество полной хаусдорфовой размерности в $\mathbb{R}^{m n+n}$.

Доказательство Д. Клейнбока было связано с рассмотрением специальных потоков на некоторых однородных пространствах. Простое доказательство этого результата дали Я. Бюжо, С. Харрап, С. Кристенсен и С. Велани в [50]. Более того, они получили более сильную теорему, которую мы сейчас и сформулируем. 
ТЕОРема 35. Для произвольного набора вещественных чисел

$$
\Theta=\left\{\theta_{j}^{i}, 1 \leqslant i \leqslant m, 1 \leqslant j \leqslant n\right\}
$$

рассмотрим множество $\mathscr{B}(\Theta)$, состоящее из векторов $\left(\eta_{1}, \ldots, \eta_{n}\right)$ таких, что

$$
\inf _{\mathbf{x} \in \mathbb{Z}^{m} \backslash\{\mathbf{0}\}}\left(\max _{1 \leqslant j \leqslant n}\left\|L_{j}(\mathbf{x})-\eta_{j}\right\|\right)^{n}\left(\max _{1 \leqslant i \leqslant m}\left|x_{i}\right|\right)^{m}>0 .
$$

Тогда $\mathscr{B}(\Theta)$ есть множество полной размерности Хаусдорфа в $\mathbb{R}^{n}$.

Для формулировки и обсуждения дальнейших результатов необходимо знание теории $(\alpha, \beta)$-игр В.М. Шмидта. Основные понятия и факты из этой теории, необходимые для понимания формулировок трех последующих теорем и дальнейших доказательств из текущего пункта, мы изложили в п. 13.4 добавления.

Сейчас мы формулируем примечательный результат, который был недавно получен Дж. Тсенгом [51].

ТЕОРемА 36. Для любого вещественного числа $\theta$ множество $\mathscr{B}$, состоящее из вещественных чисел $\eta$ таких, что

$$
\inf _{x \in \mathbb{Z} \backslash\{0\}}|x| \cdot\|\theta x+\eta\|>0,
$$

является $\alpha$-выигрышным множеством для любого $\alpha \in(0,1 / 8)$.

Согласно замечанию 2.3 из работы Дж. Тсенга [51], Дж. Тсенг и М. Айсиедлер получили обобщение теоремы 36 на случай систем линейных форм произвольной размерности (препринт [52] появился совсем недавно).

В работе [53] автор, модифицируя доказательство теоремы 33, получил следующее утверждение.

ТеОрема 37. Пусть $\alpha \in(0,1 / 2]$. Тогда для любого набора вещественных чисел

$$
\Theta=\left\{\theta_{j}^{i}, 1 \leqslant i \leqslant m, 1 \leqslant j \leqslant n\right\}
$$

множество $\mathscr{B}(\Theta)$, состоящее из векторов $\left(\eta_{1}, \ldots, \eta_{n}\right)$ таких, что

$$
\inf _{\mathbf{x} \in \mathbb{Z}^{m} \backslash\{\mathbf{0}\}}\left(\max _{1 \leqslant j \leqslant n}\left\|L_{j}(\mathbf{x})-\eta_{j}\right\|\right)^{n}\left(\max _{1 \leqslant i \leqslant m}\left|x_{i}\right|\right)^{m}>0,
$$

является $\alpha$-выигрышным множеством в $\mathbb{R}^{n}$.

Справедлив более общий результат.

Теорема 38. Пусть $\alpha \in(0,1 / 2]$. Пусть функиия $\psi(t)$ строго монотонно убывает к нулю при $t \rightarrow+\infty$, и пусть $\rho(t)$ есть функиия, обратная к функиии $1 / \psi(t)$. Предположим, что для любого $w \geqslant 1$ выполнено

$$
\sup _{x \geqslant 1} \frac{\rho(w x)}{\rho(x)}<+\infty \text {. }
$$

Для любого набора вещественных чисел $\Theta$ рассмотрим функиию Ярника $\psi_{t_{\Theta}}$. Пусть при всех достаточно больших $t$ выполнено неравенство $\psi_{t_{\Theta}}(t) \leqslant \psi(t)$. 
Тогда множество $\mathscr{B}(\Theta)$, состоящее из векторов $\left(\eta_{1}, \ldots, \eta_{n}\right)$ таких, что

$$
\inf _{\mathbf{x} \in \mathbb{Z}^{m} \backslash\{\mathbf{0}\}}\left(\max _{1 \leqslant j \leqslant n}\left\|L_{j}(\mathbf{x})-\eta_{j}\right\|\right) \rho\left(\max _{1 \leqslant i \leqslant m}\left|x_{i}\right|\right)>0
$$

является $\alpha$-выигрышным множеством в $\mathbb{R}^{n}$.

Легко видеть (так как всегда $\psi_{t_{\Theta}}(t) \leqslant t^{-n / m}$ ), что сформулированная теорема 37 является частным случаем теоремы 38 .

Ниже в этом пункте мы приводим схему доказательства последней теоремы.

Чтобы получить доказательство теоремы 38, надо всего лишь переформулировать лемму 2 о лакунарной последовательности векторов из п. 6.3 .

Лемма 3. Пусть последовательность $\Lambda \subset \mathbb{Z}^{n}$ иелочисленных векторов $\mathbf{u}^{(r)}=\left(u_{1}^{(r)}, \ldots, u_{n}^{(r)}\right) \in \mathbb{Z}^{n}$ такова, что последовательность вещественных чисел

$$
t_{r}=\left(\left(u_{1}^{(r)}\right)^{2}+\cdots+\left(u_{n}^{(r)}\right)^{2}\right)^{1 / 2}
$$

удовлетворяет условию лакунарности для некоторого $M>1$ :

$$
\frac{t_{r+1}}{t_{r}} \geqslant M, \quad r=1,2,3, \ldots
$$

Тогда множество

$$
\begin{aligned}
N(\Lambda)=\left\{\eta=\left(\eta_{1}, \ldots, \eta_{n}\right) \in \mathbb{R}^{n}: \text { существует } c(\eta)>0\right. \text { mакое, что } \\
\left.\qquad u_{1}^{(r)} \eta_{1}+\cdots+u_{n}^{(r)} \eta_{n} \| \geqslant c(\eta) \text { для любого } r \in \mathbb{N}\right\}
\end{aligned}
$$

является $\alpha$-выигрышными при каждом $\alpha \in(0,1 / 2]$.

То, что в условиях теоремы 38 всякий набор $\eta \in N(\Lambda)$ (при надлежащем выборе векторов $\mathbf{u}^{(r)}$ ) будет принадлежать множеству $\mathscr{B}(\Theta)$, сразу следует из классических аргументов доказательства теоремы 29, которые мы напомнили читателям в п. 6.3. Доказательства частного случая (теоремы 37) и общего случая (теоремы 38) не отличаются.

Ниже приводим схему доказательства леммы 3.

Как обычно, для $\alpha, \beta \in(0,1)$ полагаем $\gamma=1+\alpha \beta-2 \alpha>0$.

Рассмотрим шар (в евклидовой норме; в этом доказательстве всюду удобнее использовать именно евклидову норму) $B \subset \mathbb{R}^{n}$ с центром $O$ и радиусом $\rho$. Его границу будем обозначать $S=\partial B$. Через $\mu$ обозначаем нормализованную меру Лебега на $S$ (так что $\int_{S} d \mu=\mu S=1$ ).

Пусть $x \in S$. Определим $(n-1)$-мерное аффинное подпространство $\pi(x) \subset \mathbb{R}^{n}$ как подпространство, проходящее через точку $O$ и ортогональное одномерному подпространству, проходящему через две точки, $O$ и $x$. Определим "полупространство" П $(x)$ с границей $\pi(x)$ такое, что $x \in \Pi(x)$.

Для данных $\alpha, \beta \in(0,1)$ рассмотрим полупространство $\Pi_{\alpha, \beta, \rho}(x)$ такое, что $\Pi_{\alpha, \beta, \rho}(x) \subset \Pi(x)$ и расстояние от $\Pi_{\alpha, \beta, \rho}(x)$ до $O$ равно $\gamma \rho / 2$. Положим

$$
\Omega(x)=S \cap \Pi_{\alpha, \beta, \rho}(x), \quad \Omega^{*}(x)=\bigcup_{y \in S: \Pi(y) \supset \Omega(x)}\{y\} .
$$


Ясно, что мера $\mu \Omega^{*}(x)$ не зависит от $x \in S$. Положим

$$
\omega=\omega(\alpha, \beta)=\mu\left(\Omega^{*}(x)\right) \in(0,1) .
$$

C помощью соображений усреднения сразу получаем следующий вспомогательный результат.

ЛЕмма 4. Возъмем произвольные афбинные подпространства $\pi_{1}, \ldots, \pi_{k}$ размерности $n-1$. Тогда найдется точка $x \in S$ такая, что

$$
\Omega(x) \cap \pi_{j}=\varnothing
$$

для по крайней мере $\lceil\omega k\rceil$ индексов $j$.

Следующая лемма принадлежит В. М. Шмидту [24; гл. 3, лемма 1В].

Лемма 5. Пусть $t$ таково, что

$$
(\alpha \beta)^{t}<\frac{\gamma}{2}
$$

Предположим, что в игре встретился шар черных $B_{j}$. Предположим, что $(n-1)$-мерное аффинное подпространство $\pi$ проходит через центр шара $B_{j}$. Тогда белье могут играть таким образом, что в результате шар $B_{k+t}$ будет иеликом содержатъся в "полупространстве” $\Pi_{\alpha, \beta, \rho_{j}}(x)$, граница которого параллельна подпространству $\pi$.

Выбрав параметры

$$
t=t(\alpha, \beta)=\left\lceil\frac{\log (\gamma / 2)}{\log (\alpha \beta)}\right\rceil, \quad \tau_{k}=t\left\lceil\frac{\log k}{\log (1 /(1-\omega))}\right\rceil,
$$

где $\omega$ определено в (84), получаем следующее утверждение.

СлЕДСТвИЕ. Пусть шар $B_{j}$ с радиусом $\rho_{j}$ встретился в игре черных, $u$ пусть имеется набор афбинных подпространств $\pi_{i}, 1 \leqslant i \leqslant k$. Тогда белье могут играть таким образом, что для каждой точки $x \in B_{j+\tau_{k}}$ расстояние от точки $x$ до каждого из подпространств $\pi_{i} c 1 \leqslant i \leqslant k$ будет больше, чем $\left(\rho_{j+\tau_{k}} \gamma\right) / 2$.

Далее выбираем параметр $k=k(\alpha, \beta, M)$ такой, что

$$
\tau_{k} \frac{\log (1 /(\alpha \beta))}{\log M}+2<k
$$

Не ограничивая общности, мы можем предполагать, что в дополнение к условию лакунарности (83) выполняются неравенства $t_{r+1} / t_{r} \leqslant M^{2}$ при $r=$ $1,2,3, \ldots$. Выбор параметров (85), (86) позволяет белым вести игру таким образом, что за время игры $\tau_{k}$ удавалось бы "убегать" от $q=r_{j-1}-r_{j}<k$ семейств "опасных" подпространств вида

$$
\left\{\mathbf{y}=\left(y_{1}, \ldots, y_{n}\right) \in \mathbb{R}^{n}: u_{1}^{(r)} y_{1}+\cdots+u_{n}^{(r)} y_{n}=a\right\}, \quad a \in \mathbb{Z}, \quad r_{j} \leqslant r<r_{j+1} .
$$




\section{7. Пространства решеток}

Задачи о диофантовых приближениях естественным образом связаны с вопросами поведения семейств (орбит) некоторых решеток. На эту тему имеется много работ (см., например, работы Г. А. Маргулиса, Д. Клейнбока [49] и Б. Вайса [54]-[57], обзор А. Городника [58] и литературу, цитируемую в этих работах). Ниже мы остановимся на двух задачах, имеющих отношение к сингулярным матрицам.

7.1. Метрическая теорема Давенпорта-Шмидта. Как отмечалось в конце п. 1.2, сингулярные системы $\Theta$ образуют в $n m$-мерном пространстве множество нулевой меры Лебега. То же самое можно сказать так. Рассмотрим множество $\mathfrak{T}_{\mu} \subset \mathbb{R}^{m n}$, состоящее из тех наборов $\Theta$, для которых при всех достаточно больших значениях параметра $t$ в каждой области

$$
\left\{\mathrm{x} \in \mathbb{R}^{n}: \max _{1 \leqslant j \leqslant n}\left\|L_{j}(\mathbf{x})\right\| \leqslant \frac{\mu}{t}, 0<\max _{1 \leqslant i \leqslant m}\left|x_{i}\right| \leqslant \mu t^{n / m}\right\}
$$

имеется ненулевая целая точка. (Ясно, что $\mathfrak{T}_{\mu_{1}} \subset \mathfrak{T}_{\mu_{2}}$ при $\mu_{1}<\mu_{2}$.) Тогда пересечение

$$
\bigcap_{\mu>0} \mathfrak{T}_{\mu}
$$

имеет лебегову меру нуль.

Замечательное усиление этого факта было получено Г. Давенпортом и В. М. Шмидтом в [39], [25].

Теорема 39 (Г. Давенпорт, В. М. Шмидт [39], [25]). Для любого и из интервала $0<\mu<1$ множество $\mathfrak{T}_{\mu}$ имеет меру нуль.

Отметим, что согласно теореме Минковского о выпуклом теле, упоминавшейся в самом начале нашей работы, при $\mu=1$ имеем $\mathfrak{T}_{1}=\mathbb{R}^{m n}$.

ЗАмечАнИЕ. На самом деле, в работах [29], [30] рассмотрен только случай $n=1$ (одна линейная форма) или $m=1$ (совместные приближения); тем не менее, доказательства проходят в общем случае. В случае же, когда и $m=1$ и $n=1, \Gamma$. Давенпорт и В.М. Шмидт установили, что из условия $\theta \in \mathfrak{T}_{\mu}$ с некоторым $\mu<1$ вытекает, что $\theta$ является плохо приближаемым числом, т. е.

$$
\liminf _{q \rightarrow \infty} q\|q \theta\|>0
$$

(в последней формуле, естественно, предполагается, что $q$ принимает целые значения), или, что то же самое, неполные частные разложения $\theta$ в цепную дробь ограничены. Фактически этот результат связан с применением формулы (46).

Доказательство теоремы 39 в случае $n=1$ фактически основывается на следующем утверждении.

Теорема 40 (В. М. Шмидт [59]). Рассмотрим последовательность натуральных чисел $N_{\nu}$, возрастающих $\kappa$ бесконечности. Для набора вещественных чисел $\Theta=\left(\theta^{1}, \ldots, \theta^{m}\right) \in \mathbb{R}^{m}$ рассмотрим решетку

$$
\Lambda(\Theta, N)=\mathscr{A}(\Theta, N) \mathbb{Z}^{m+1},
$$


где матрица $\mathscr{A}(\Theta, N)$ имеет вид

$$
\mathscr{A}(\Theta, N)=\left(\begin{array}{ccccc}
N^{-1} & 0 & 0 & \ldots & 0 \\
0 & N^{-1} & 0 & \ldots & 0 \\
0 & 0 & N^{-1} & \ldots & 0 \\
\ldots \ldots \ldots \ldots \ldots \ldots \ldots \ldots \ldots \ldots & \ldots \ldots \\
\theta^{1} N^{m} & \theta^{2} N^{m} & \theta^{3} N^{m} & \ldots & N^{m}
\end{array}\right) .
$$

Тогда для почти всех (в смысле меры Лебега) наборов $\Theta \in \mathbb{R}^{m}$ последовательность решеток

$$
\Lambda\left(\Theta, N_{\nu}\right), \quad \nu=1,2,3, \ldots,
$$

всюду плотна в пространстве решеток в $\mathbb{R}^{m+1}$ с определителем 1 .

Случай $m=1$, связанный с рассмотрением решеток вида

$$
\begin{aligned}
& \Lambda^{*}(\Theta, N)=\mathscr{A}^{*}(\Theta, N) \mathbb{Z}^{n+1}, \\
& \mathscr{A}^{*}(\Theta, N)=\left(\begin{array}{ccccc}
N^{-1} & 0 & 0 & \ldots & 0 \\
N^{1 / n} \theta_{1} & N^{1 / n} & 0 & \ldots & 0 \\
N^{1 / n} \theta_{2} & 0 & N^{1 / n} & \ldots & 0 \\
\ldots \ldots \ldots \ldots \ldots \ldots \ldots \ldots \ldots \ldots \ldots \ldots \ldots \\
N^{1 / n} \theta_{n} & 0 & 0 & \ldots & N^{1 / n}
\end{array}\right), \quad \Theta=\left(\begin{array}{c}
\theta_{1} \\
\theta_{2} \\
\vdots \\
\theta_{n}
\end{array}\right),
\end{aligned}
$$

в оригинальной работе [25] получается из случая $n=1$ с помощью техники переноса.

В настоящей статье мы не будем вдаваться в подробности, касающиеся пространства решеток и вопросов сходимости в нем. Отметим лишь, что классические работы K. Малера [60] и [61] на эту тему изложены в [62; гл. V]. Следует также сказать, что ряд результатов теории диофантовых приближений оказался связанным с рассмотрением специальных динамических систем на пространствах решеток и получил свое развитие в работах Д. Клейнбока. В частности, в работе [57] получено обобщение теоремы Давенпорта-Шмидта, о которой идет речь в настоящем пункте.

7.2. Задача о последовательных минимумах. Пусть даны решетка $\Lambda \subset \mathbb{R}^{d}$ и выпуклое 0-симметричное тело $\Omega \subset \mathbb{R}^{d}$. Величины

$$
\begin{gathered}
\mu_{l}(\Omega, \Lambda)=\inf \{t: t \Omega \text { содержит } l \text { линейно независимых точек решетки } \Lambda\}, \\
1 \leqslant l \leqslant d,
\end{gathered}
$$

называются последовательными минимумами решетки $\Lambda$ относительно тела $\Omega$. Знаменитая вторая теорема Минковского о выпуклом теле (см., например, [62; гл. VIII] или [24; гл. IV]) утверждает, что

$$
\frac{2^{d}}{d !} \operatorname{det} \Lambda \leqslant \mu_{1}(\Omega, \Lambda) \cdots \mu_{d}(\Omega, \Lambda) \cdot \operatorname{mes} \Omega \leqslant 2^{d} \operatorname{det} \Lambda .
$$

В настоящем пункте мы ограничимся рассмотрением задачи о совместных приближениях, т. е. случаем $m=1$. Теорема Минковского о линейных формах, упоминавшаяся в начале нашей работы, может быть переформулирована в этом 
случае следующим образом. Рассмотрим куб $E=[-1,1]^{n+1} \subset \mathbb{R}^{n+1}$ и для набора $\Theta$ решетку $\Lambda^{*}(\Theta, N)$, определенную в (88). Тогда для любого вещественного $N \geqslant 1$

$$
\mu_{1}\left(E, \Lambda^{*}(\Theta, N)\right) \leqslant 1
$$

Как отмечал В. М. Шмидт [63], легко видеть, что для каждого $k, 1 \leqslant k \leqslant n$, найдется последовательность вещественных чисел $N_{\nu}$, стремящаяся к бесконечности, такая, что

$$
\mu_{k}\left(E, \Lambda^{*}\left(\Theta, N_{\nu}\right)\right)=\mu_{k+1}\left(E, \Lambda^{*}\left(\Theta, N_{\nu}\right)\right)
$$

(Более общее утверждение и приложения к затронутым в настоящей статье вопросам, в частности, следствия для теории переноса, имеются в недавней работе В. М. Шмидта и Л. Саммерера [64].) В частности, при $n=1$ из теоремы Минковского о последовательных минимумах получаем, что

$$
1 \ll \mu_{1}\left(E, \Lambda^{*}(\Theta, N)\right) \mu_{2}\left(E, \Lambda^{*}(\Theta, N)\right) \ll 1,
$$

и, следовательно, не может выполняться равенство

$$
\lim _{N \rightarrow+\infty} \mu_{1}\left(E, \Lambda^{*}(\Theta, N)\right)=0 .
$$

Но если $n>1$, то даже для линейно независимых вместе с единицей чисел $\theta_{1}, \ldots, \theta_{n}$ может оказаться, что

$$
\lim _{N \rightarrow+\infty} \mu_{n-1}\left(E, \Lambda^{*}(\Theta, N)\right)=0 .
$$

(Набор $\Theta$ в этом случае следует брать из теоремы 4, с надлежащим выбором функции $\psi$.)

Автор в [65] доказал следующую теорему, ответив тем самым на вопрос, поставленный В. М. Шмидтом [63].

Tеорема 41. Пусть $n \geqslant 2 u 1 \leqslant k \leqslant n-1$. Тогда найдется набор $\Theta=$ $\left(\theta_{1}, \ldots, \theta_{n}\right)$ вещественных чисел такой, что

- числа $1, \theta_{1}, \ldots, \theta_{n}$ линейно независимы над $\mathbb{Z}$;

- выполнено $\mu_{k}(\xi, N) \rightarrow 0$ при $N \rightarrow+\infty$;

- выполнено $\mu_{k+2}(\xi, N) \rightarrow+\infty$ при $N \rightarrow+\infty$.

Доказательство этой теоремы в общем случае достаточно громоздко. В случае $k=1$ доказательство весьма просто. Оно идейно очень похоже на доказательство теоремы 18.

ЗАмечАниЕ. Легко видеть, что утверждение теоремы 41 становится тривиальным, если не требовать выполнения условия линейной независимости над $\mathbb{Z}$.

Отметим в заключение этого раздела, что недавно усиление теоремы 41 анонсировал И. Чеунг. 


\section{8. Теоремы переноса}

Теоремы переноса - это утверждения о том, что если система $\Theta$ обладает некоторыми диофантовыми свойствами, то и транспонированная система ${ }^{t} \Theta$ тоже обладает некоторыми диофантовыми свойствами. Простейшая теорема переноса, касающаяся сингулярных систем, уже упоминалась нами в п. 6.2 (теорема 31). Утверждения о связи однородной задачи для системы ${ }^{t} \Theta$ и неоднородной задачи для системы $\Theta$ (такие, как теоремы 27-33) тоже можно интерпретировать как теоремы переноса. Теории переноса посвящено довольно много работ. Ниже мы перечисляем некоторые виды теорем переноса. Список никоим образом не претендует на полноту, но тем не менее дает некоторое представление о том, какие математики и в связи с какими вопросами занимались переносом (более подробную библиографию можно почерпнуть как раз из упоминаемых ниже работ).

- Классические результаты А. Я. Хинчина о диофантовых экспонентах для "обычных" диофантовых приближений [2], [1]; общий случай, рассмотренный Ф. Дайсоном [66]; точность оценок А. Я. Хинчина, доказанная В. Ярником [15].

- Общие конструкции К. Малера [67].

- Теоремы переноса для сингулярных систем (работы В. Ярника [9], А. Апфельбека [29], М. Лорана и Я. Бюжо [43], [68], [69]; здесь же уместно упомянуть недавнюю работу В. М. Шмидта и Л. Саммерера [64]).

- Теоремы переноса для приближений рациональных чисел (Н. М. Коробов [70], [71]); интерес к такого рода задачам появился в связи с теорией приближенного интегрирования.

- Теоремы переноса, так или иначе связанные с применением тригонометрических сумм. Они имеются, например, у А. О. Гельфонда [72] и Ю. В. Каширского [73]. Этот подход основывается на работе К. Л. Зигеля [74].

- Теоремы переноса для произведений линейных форм (В.М. Шмидт и Ю. Ван [75], а также Ю. В. Каширский [73]).

- Более тонкие результаты типа пареноса, связанные с приближениями рациональными подпространствами (работы В. М. Шмидта [76], М. Лорана [43], М. Лорана и Я. Бюжо [69]).

Классические результаты изложены в книгах Дж. В. С. Касселса [23] (глава V) и В. М. Шмидта [24] (глава IV).

В настоящей работе нас наиболее интересуют теоремы переноса для сингулярных систем, т. е. связанные с поведением величин типа

$$
\limsup _{t \rightarrow+\infty} \varphi(t) \psi_{\Theta}(t)
$$

где $\varphi(t)$ есть некоторая функция, а $\psi_{\Theta}$ есть функция Ярника (20). Это результаты В. Ярника и А. Апфельбека и теоремы, недавно полученные М. Лораном и Я. Бюжо.

Но мы начнем с того, что приведем классические теоремы А.Я. Хинчина и Ф. Дайсона.

Прежде чем формулировать результаты, напомним определение экспоненты $\alpha(\Theta)$ - это супремум тех $\gamma$, для которых выполнено

$$
\limsup _{t \rightarrow+\infty} t^{\gamma} \psi_{\Theta}(t)<+\infty .
$$


Здесь же напомним определение экспоненты $\beta(\Theta)$, которая есть супремум тех $\gamma$, для которых выполнено

$$
\liminf _{t \rightarrow+\infty} t^{\gamma} \psi_{\Theta}(t)<+\infty
$$

(эти определения уже использовались у нас в статье в разделе 5).

8.1. Теоремы А. Я. Хинчина и Ф. Дайсона. Теорему переноса для показателей $\beta(\Theta)$ для системы $\Theta=\left(\theta^{1}, \ldots, \theta^{m}\right) \in \mathbb{R}^{m}$ и транспонированной системы ${ }^{t} \Theta$ А. Я. Хинчин доказал все в той же знаменитой работе [1].

Tеорема 42. Для набора $\Theta=\left(\theta^{1}, \ldots, \theta^{m}\right) \in \mathbb{R}^{m}$ имеют место неравенства

$$
\frac{\beta(\Theta)}{(m-1) \beta(\theta)+m} \leqslant \beta\left({ }^{t} \Theta\right) \leqslant \frac{\beta(\theta)-m+1}{m} .
$$

Результат Ф. Дайсона [66] (простое доказательство есть у А.Я. Хинчина в [8]) выглядит так:

ТЕОРема 43. При произвольных значениях размерностей $m$ и $n$ для матрицы $\Theta$ выполнено неравенство

$$
\beta\left({ }^{t} \Theta\right) \geqslant \frac{n \beta(\Theta)+n-1}{(m-1) \beta(\Theta)+m} .
$$

Естественно, теорему 42 можно получить, применив два раза теорему 43 (для матрицы-строки и для матрицы-столбца).

8.2. Результаты В. Ярника и А. Апфельбека. Работа В. Ярника [9] посвящена теоремам переноса, связывающим случаи $m=1$ и $n=1$. Мы приведем общий результат (теоремы 44, 45; в оригинальной работе [9] - Satz 7 и Satz 8) и перечислим некоторые его следствия. Будем рассматривать вектор-строку

$$
\Theta=\left(\theta^{1}, \ldots, \theta^{m}\right), \quad m \geqslant 2,
$$

и функции

$$
\psi_{\Theta}(t), \quad \psi_{t_{\Theta}}(t) .
$$

Теорема 44. Пусть числа $1, \theta^{1}, \ldots, \theta^{m}$ линейно независимы над $\mathbb{Z}$, и $K$ некоторая положительная постоянная. Предположим, что функиия $\varphi(t)$ возрастает, а функция $t \mapsto t(\varphi(t))^{-1}$ монотонно возрастает $\kappa$ бесконечнос$m u$ u

$$
\limsup _{t \rightarrow+\infty} \varphi(t) \psi_{t_{\Theta}}(t)<K
$$

Тогда:

(i) выполняется неравенство

$$
\limsup _{t \rightarrow+\infty} t^{m-1} \varphi\left(t^{m}\right) \psi_{\Theta}(t) \leqslant m^{2 m} K
$$

(ii) если же известно, что $\varphi(t) \geqslant t^{(m-1) / m}$ при всех достаточно больших значениях $t, u$ функция $\rho(t)$ есть обратная $к$ функции $t \mapsto t(\varphi(t))^{-1}$, то

$$
\limsup _{t \rightarrow+\infty} t^{m-2} \rho\left(\frac{t}{2 K}\right) \psi_{\Theta}(t) \leqslant m^{m}(K+1) .
$$


ТеОРема 45. Пусть числа $1, \theta^{1}, \ldots, \theta^{m}$ линейно независимы над $\mathbb{Z}$ и $K$ есть некоторая положительная постоянная. Предположим, что функиия $\varphi(t)$ возрастает $\kappa+\infty$ при $t \rightarrow+\infty, \rho(t)$ есть функция, обратная $\kappa$ функции $t \mapsto t \varphi^{(m-1) / m}(t)$, a также

$$
\limsup _{t \rightarrow+\infty} \varphi(t) \psi_{\Theta}(t)<K
$$

Тогда:

(i) выполняется неравенство

$$
\limsup _{t \rightarrow+\infty}\left(\frac{t}{\rho\left(t K^{(m-1) / m}\right)}\right)^{1 /(m-1)} \psi_{t_{\Theta}}(t) \leqslant 3 m^{2}(K+1) ;
$$

(ii) если $\varphi(t) \geqslant t^{m(2 m-3)}$ и если при достаточно больиих значениях $t$ функиия $\varphi(t) t^{-2 m+3}$ возрастает, а функиия $\rho_{1}(t)$ есть обратная $\kappa$ ней, то

$$
\limsup _{t \rightarrow+\infty}\left(\frac{t}{\rho_{1}(t K)}\right)^{1 /(m-1)} \psi_{t_{\Theta}}(t) \leqslant 3 m^{2}(K+1) .
$$

Отдельно обсудим случай $m=2$. В этом случае, согласно (18), имеем

$$
\psi_{t_{\Theta}}(t) t^{1 / 2} \leqslant 1, \quad \psi_{\Theta}(t) t^{2} \leqslant 1
$$

Таким образом, следует использовать утверждение (ii) теоремы 44 и утверждение (ii) теоремы 45.

СлеДСтвие 1 [9; Satz 2]. (i) Пусть функиия $\varphi(t)$ такова, что функция $t \mapsto t^{-1} \varphi(t)$ возрастает, при достаточно больших $t$ выполняется неравенство $\varphi(t) \geqslant t^{2} u$

$$
\limsup _{t \rightarrow+\infty} \varphi(t) \psi_{\Theta}(t)<K
$$

Тогда для функиии $\rho(t)$, обратной $\kappa \varphi(t)$, выполнено

$$
\limsup _{t \rightarrow+\infty} \frac{t}{\rho(t K)} \psi_{t_{\Theta}}(t)<12(K+1) .
$$

(ii) Пусть функиия $\varphi(t)$ такова, что функиия $t \mapsto t \varphi(t)^{-1}$ возрастает, при достаточно больших $t$ выполняется неравенство $\varphi(t) \geqslant t^{1 / 2} u$

$$
\limsup _{t \rightarrow+\infty} \varphi(t) \psi_{t_{\Theta}}(t)<K
$$

Тогда для функиии $\rho(t)$, обратной $\kappa$ функции $t \mapsto t \varphi(t)^{-1}$, выполнено

$$
\limsup _{t \rightarrow+\infty} \rho\left(\frac{t}{2 K}\right) \psi_{\Theta}(t)<4(K+1) .
$$

Последнее следствие наиболее известно в качестве утверждения о диофантовых экспонентах. С учетом определения величины $\alpha(\Theta)$ следствие 1 дает следующий результат. 
СлЕДСтвиЕ 2 [9; Satz 1]. При $m=2$ выполнено равенство

$$
\alpha(\Theta)=\frac{1}{1-\alpha\left({ }^{t} \Theta\right)} .
$$

Отметим, что А. Я. Хинчин в работе [8] приводит простое и короткое доказательство равенства В. Ярника (91).

Приведем следствие теорем 44, 45, касающееся диофантовых экспонент $\alpha(\Theta), \alpha\left({ }^{t} \Theta\right)$ в случае произвольного значения размерности $m$.

СледСтвиЕ 3 [9; Satz 3]. (i) Всегда выполняются неравенства

$$
\begin{gathered}
\alpha(\Theta) \geqslant(m-1)+m \alpha\left({ }^{t} \Theta\right), \\
\alpha\left({ }^{t} \Theta\right) \geqslant \frac{1}{m-1}\left(1-\frac{m}{(m-1) \alpha(\Theta)+m}\right)=\frac{\alpha(\Theta)}{(m-1) \alpha(\Theta)+m} .
\end{gathered}
$$

(ii) $\operatorname{Ecли~} \alpha\left({ }^{t} \Theta\right)>(m-1) / m, m o$

$$
\alpha(\Theta) \geqslant m-2+\frac{1}{1-\alpha\left({ }^{t} \Theta\right)} .
$$

(ii) $\operatorname{Ecлu~} \alpha(\Theta)>m(2 m-3)$, mo

$$
\alpha\left({ }^{t} \Theta\right) \geqslant \frac{1}{m-1}\left(1-\frac{1}{\alpha(\Theta)-2 m+4}\right) .
$$

Естественно, следствие 2 можно получить, положив $m=2$ в следствии 3 .

А. Апфельбек [29] обобщил теоремы 44, 45 на случай произвольных $m$ и $n$.

Теорема 46 (А. Апфельбек [29]). Пусть матрица $\Theta$ невырождена, $K$ положительное число, функиия $\varphi(t)$ возрастает $\kappa+\infty$ при $t \rightarrow+\infty u$

$$
\limsup _{t \rightarrow+\infty} \varphi(t) \psi_{\Theta}(t)<K
$$

Тогда:

(i1) при $m=1$ выполняется неравенство

$$
\limsup _{t \rightarrow+\infty} t^{n-1} \varphi\left(\frac{t^{n}}{2(n-1)}\right) \psi_{t_{\Theta}}(t) \leqslant 2(n+1) K
$$

(i2) при $m>1$ выполняется неравенство

$$
\limsup _{t \rightarrow+\infty}\left(\frac{t^{n}}{\rho\left(K^{(m-1) /(m+n-1)} t\right)}\right)^{1 /(m-1)} \psi_{t_{\Theta}}(t) \leqslant(2(n+m))^{1 /(m-1)},
$$

где $\rho(t)$ обозначает функиию, обратную к функиии

$$
t \mapsto\left(t^{m}(\varphi(t))^{m-1}\right)^{1 /(m+n-1)} ;
$$

(ii) если же при $m>1$ известно, что

$$
\varphi(t) \geqslant 2^{m+n-2} K t^{\left(2(m+n-2)^{2}+m-2\right) / n}
$$


при всех достаточно больших значениях $t$ и функция $t \mapsto t^{-(2 m+n-1) / n} \varphi(t)$ возрастает, то выполнено

$$
\limsup _{t \rightarrow+\infty}\left(\frac{t^{n}}{\rho_{1}\left(K^{(m-1) /(m+n-2)} t\right)}\right)^{1 /(m-1)} \psi_{\Theta}(t) \leqslant 3(m+n),
$$

где $\rho_{1}(t)$ обозначает функцию, обратную к функиии

$$
t \mapsto\left(t^{-(m-2)(2 m+n-3) /((m-1) n)} \varphi(t)\right)^{(m-1) /(m+n-2)} .
$$

Ясно, что из теоремы 46 получается следствие о диофантовых экспонентах $\alpha(\Theta), \alpha\left({ }^{t} \Theta\right)$.

СлЕДСТвИЕ [29; теорема 6]. (i) При произвольных $m$ и $n$ имеет место неравенство

$$
\alpha\left({ }^{t} \Theta\right) \geqslant \frac{n \alpha(\Theta)+n-1}{(m-1) \alpha(\Theta)+m} .
$$

(ii) Если $m>1$ и известно, что

$$
\alpha(\Theta)>\frac{2(m+n-1)(m+n-3)+m}{n},
$$

mo

$$
\alpha\left({ }^{t} \Theta\right) \geqslant \frac{1}{m}\left(n+\frac{n(n \alpha(\Theta)-m)-2 n(m+n-3)}{(m-1)(n \alpha(\Theta)-m)+m-(m-2)(m+n-3)}\right) .
$$

А. Апфельбек в работе [29] доказал также, что в случае $\alpha(\Theta)=+\infty$ неравенство $\alpha\left({ }^{t} \Theta\right) \geqslant n /(m-1)$ (которое вытекает из следствия теоремы 46) является точным (теорема 11 из [29], ее доказательство основывается на построении сингулярных матриц $\Theta$ специального вида).

8.3. Теоремы М. Лорана. В работе [43] М. Лоран получает следующий результат.

ТЕОРема 47. Для экспонент двумерных диобантовых приближений имеет место следующее.

(i) Для вектор-строки $\Theta=\left(\theta^{1}, \theta^{2}\right) \in \mathbb{R}^{2}$ такой, что $\operatorname{dim}_{\mathbb{Z}} \Theta=3$, для величин

$$
w=\alpha(\Theta), \quad w^{*}=\alpha\left({ }^{t} \Theta\right), \quad v=\beta(\Theta), \quad v^{*}=\beta\left({ }^{t} \Theta\right)
$$

(определения которых мы приводили и в $n .5 .3$, и в $n .8 .2$ ) выполнены соотношения

$$
2 \leqslant w \leqslant+\infty, \quad w=\frac{1}{1-w^{*}}, \quad \frac{v(w-1)}{v+w} \leqslant v^{*} \leqslant \frac{v-w+1}{w} .
$$

(ii) Для произвольного набора из четырех чисел $\left(w, w^{*}, v, v^{*}\right)$, удовлетворяющего соотношениям (93), найдется вектор-строка $\Theta=\left(\theta^{1}, \theta^{2}\right) \in \mathbb{R}^{2}$, $\operatorname{dim}_{\mathbb{Z}} \Theta=3$, такая, что выполнено (92). 
Скажем несколько слов о теореме 47. Первое соотношение из (93) есть следствие теоремы Минковского (18); второе соотношение есть результат В. Ярника - следствие 2 теорем 44, 45; третье соотношение доказывается М. Лораном с помощью рассмотрения последовательностей наилучших приближений и с использованием соотношения В. Ярника (91). Принадлежащие В. Ярнику следствие теоремы 19 из п. 5.1 и следствие теоремы 21 из п. 5.3 (естественно, при $m=1, n=2$ и $m=2, n=1$ соответственно) могут быть получены из утверждения (i) теоремы 47. Утверждение (ii) теоремы 47, в частности, показывает, что следствия теорем В. Ярника из пп. 5.1, 5.3 при $m=1, n=2$ и $m=2$, $n=1$ содержат точные (неулучшаемые) оценки. Более того, из теоремы 47 вытекает, что пары величин $(\alpha(\Theta), \beta(\Theta))$ и $\left(\alpha\left({ }^{t} \Theta\right), \beta\left({ }^{t} \Theta\right)\right)$ могут принимать любые допустимые (т. е. согласующиеся с неравенствами (93)) значения.

Обобщение третьего неравенства из (93) на случай одной линейной формы от $m$ переменных имеется в работе М. Лорана [68]. Оно выглядит следующим образом.

ТЕОремА 48. Для вектор-строки $\Theta=\left(\theta^{1}, \ldots, \theta^{m}\right), m \geqslant 2$, с условием $\operatorname{dim}_{\mathbb{Z}} \theta=m+1$ выполняются неравенства

$$
\begin{gathered}
\frac{(\alpha(\Theta)-1) \beta(\Theta)}{((m-2) \alpha(\Theta)+1) \beta(\Theta)+(m-1) \alpha(\Theta)} \leqslant \beta\left({ }^{t} \Theta\right) \\
\leqslant \frac{\left(1-\alpha\left({ }^{t} \Theta\right)\right) \beta(\Theta)-m+2-\alpha\left({ }^{t} \Theta\right)}{m-1} .
\end{gathered}
$$

В свете равенства В. Ярника (91) при $m=2$ соотношения (94) совпадают с третьим соотношением из (93).

Из (18), очевидно, получаем

$$
\alpha(\Theta) \geqslant m, \quad \alpha\left({ }^{t} \Theta\right) \geqslant \frac{1}{m} .
$$

В случае, когда в обоих неравенствах (95) имеет место равенство, неравенство М. Лорана (94) совпадает с неравенством А. Я. Хинчина (89). Если же известно, что неравенства в (95) строгие, то неравенство М. Лорана (94) будет сильнее, чем неравенство А.Я. Хинчина (89). Таким образом, теорема 48 утверждает, что теорема 42 может быть усилена для сингулярных наборов $\Theta$. Автору неизвестно, имеется ли аналогичное усиление теоремы Ф. Дайсона (имеется в виду теорема 43), связанное с рассмотрением сингулярных матриц $\Theta$. Ясно, что такого рода результат должен иметь место.

Уточнение приведенных результатов М. Лорана имеется у Я. Бюжо и М. Лорана [69].

\section{9. О размерности Хаусдорфа множеств сингулярных систем}

О точных значениях и оценках размерности Хаусдорфа множеств сингулярных матриц известно не так много. Ниже мы перечислим некоторые результаты. Отметим, что ряд задач, связанных с отысканием размерностей Хаусдорфа, поставлен Я. Бюжо и М. Лораном [77]. Насколько известно автору, в основном исследовался случай $n=1$, причем получены только оценки 
размерности Хаусдорфа. Исключением является работа И. Чеунга, в которой получен следующий замечательный результат.

Теорема 49 (И. Чеунг [78]). Хаусдорфова размерность сингулярных систем (в смысле определения Хинчина при $n=2, m=1$ ) вида $\left(\begin{array}{l}\theta_{1} \\ \theta_{2}\end{array}\right)$ равна 4/3.

Из принципа переноса следует, что в теореме 49 можно говорить о хаусдорфовой размерности сингулярных систем $\left(\theta^{1}, \theta^{2}\right), n=1, m=2$.

Далее мы будем рассматривать множества $E_{m}(\alpha)$ сингулярных векторов $\Theta=\left(\theta^{1}, \ldots, \theta^{m}\right)$ (т. е. случай произвольного $m \geqslant 2$ и $n=1$ ), определенные следующим образом:

$$
E_{m}(\alpha)=\left\{\Theta \in \mathbb{R}^{m}: \lim _{t \rightarrow+\infty} t^{\alpha} \psi_{\Theta}(t)=0\right\}
$$

Сформулируем наиболее точные известные автору оценки снизу и сверху хаусдорфовой размерности.

Следующий результат принадлежит Р. К. Бейкеру [79]; он улучшает предшествующие результаты Р. К. Бейкера [80], К. Ю. Явида [81] (он первый привел контрпример к предположению Р. К. Бейкера о том, что хаусдорфова размерность множества сингулярных векторов чрезвычайно мала, сформулированному в [80], однако затем результат К. Ю. Явида был количественно улучшен Б. Ринном) и Б. Ринна [82].

Теорема 50 (Р.К. Бейкер [79]). При $m \geqslant 3 u \alpha>m$ для размерности Хаусдорфа множества $E_{m}(\alpha)$ выполняется оченка снизу

$$
\mathrm{HD} E_{m}(\alpha) \geqslant m-2+\frac{m}{\alpha} \text {. }
$$

Оценку сверху получил Б. Ринн [83], улучшив результат Р. К. Бейкера из [80].

Теорема 51 (Б. Ринн [83]). При $m \geqslant 3 u \alpha>m$ для размерности Хаусдорба множества $E_{m}(\alpha)$ выполняется оценка сверху

$$
\text { HD } E_{m}(\alpha) \leqslant m-2+\frac{2 m+2}{\alpha+1} .
$$

В случае $m=2$ обе наилучшие оценки (верхняя имеется в [79], нижняя следует из результата работы [80]) некоторое время принадлежали Р. К. Бейкеру:

Теорема 52 (Р. К. Бейкер [80], [79]). Для размерности Хаусдорфа множества $E_{2}(\alpha)$ выполняется двусторонняя оценка

$$
\frac{2}{\alpha} \leqslant \operatorname{HD} E_{2}(\alpha) \leqslant \frac{6}{\alpha+1}
$$

Нижняя оценка теоремы 52, по-видимому, является наилучшей известной до сих пор.

Применяя равенство В. Ярника (91), неравенство (58) из следствия теоремы 19 (тоже принадлежащее В. Ярнику) и частный случай $(n=2, m=1)$ 
утверждения, имеющегося в работе М. М. Додсона [84] (теорема 53 ниже), Я. Бюжо и М. Лоран в работе [77] выводят неравенство

$$
\operatorname{HD} E_{2}(\alpha) \leqslant \frac{3 \alpha}{\alpha^{2}-\alpha+1},
$$

более сильное, чем верхняя оценка из теоремы 52. Точнее, в [77] доказано, что

$$
\mathrm{HD}\left\{\Theta \in \mathbb{R}^{2}, \alpha(\Theta) \geqslant \alpha\right\} \leqslant \frac{3 \alpha}{\alpha^{2}-\alpha+1} .
$$

Приведем общую формулировку использованного результата из [84]. Напомним, что показатель $\beta(\Theta)$ обозначает супремум тех $\gamma$, для которых

$$
\liminf _{t \rightarrow+\infty} t^{\gamma} \psi_{\Theta}(t)<+\infty .
$$

ТЕОРема 53. При каждом $\tau>m / n$ размерность Хаусдорфа множества

$$
\mathfrak{W}_{m, n}(\tau)=\{\Theta: \beta(\Theta) \geqslant \tau\}
$$

равна

$$
\operatorname{HD} \mathfrak{W}_{m, n}(\tau)=(m-1) n+\frac{m+n}{\tau+1} .
$$

Приведем еще два простых следствия из сформулированных выше результатов. Из теорем 50 и 51 для множества

$$
E_{m}(\infty)=\bigcap_{\alpha>m} E_{m}(\alpha)
$$

выводим следующий результат.

СлЕДСТвИЕ 1. Для размерности Хаусдорфа множества $E_{m}(\infty)$ имеет место равенство

$$
\text { HD } E_{m}(\infty)=m-2 .
$$

Отметим также, что из теоремы 50 получается следующее утверждение.

СлеДСтвиЕ 2. Размерность Хаусдорфа множества всех сингулярных (в смысле определения А.Я. Хинчина) при $n=1$ и произвольном $m \geqslant 2$ оценивается снизу величиной $m-1$.

В заключение настоящего раздела хочется сослаться на книги В. И. Берника и Ю. В. Мельничука [85] и В. И. Берника и М. М. Додсона [86], посвященные диофантовым приближениям и размерности Хаусдорфа.

\section{0. Приближения с целыми неотрицательными числами}

10.1. Двумерные приближения. Положим $\tau=(1+\sqrt{5}) / 2$. В работе [87] В. М. Шмидт получил следующий результат.

ТЕОРема 54. Пусть вещественные числа $\theta^{1}$ и $\theta^{2}$ линейно независимы вместе с единицей над $\mathbb{Z}$. Тогда найдется последовательность иельх двумерньх векторов $\left(x_{1}(i), x_{2}(i)\right)$ таких, что:

1) $x_{1}(i), x_{2}(i)>0$

2) $\left\|\theta^{1} x_{1}(i)+\theta^{2} x_{2}(i)\right\|\left(\max \left\{x_{1}(i), x_{2}(i)\right\}\right)^{\tau} \rightarrow 0$ npu $i \rightarrow+\infty$. 
Известная гипотеза (см. [87], [63]) о том, что показатель $\tau$ в теореме 54 может быть заменен на $2-\varepsilon$ с произвольным сколь угодно малым $\varepsilon$, до сих пор не доказана. Тем не менее, ряд математиков получили обобщения и уточнения теоремы В. М. Шмидта (см., например, [88]-[90]). Доказательство В. М. Шмидта использовало утверждение о независимости наилучших приближений линейной формы (теорема $7, m=2$ и $n=1$ ) и фактически было связано с разбором двух случаев, различающихся в зависимости от того, насколько сингулярным является набор $\theta^{1}, \theta^{2}(n=1, m=2)$. Фактически, надо разбирать два случая. Первый случай, когда для всех $\nu$ таких, что наилучшие приближения $\mathbf{z}_{\nu-1}$, $\mathbf{z}_{\nu}, \mathbf{z}_{\nu+1}$ независимы, выполнено $\zeta_{\nu} \leqslant M_{\nu}^{1 / \tau} M_{\nu+1}^{-\tau /(\tau-1)}$. Второй случай, когда для бесконечно многих $\nu$ выполняется противоположное неравенство.

В [91] был разобран случай плохо приближаемых чисел $\theta^{1}, \theta^{2}$. Сформулируем результат из [91]. Для вещественного $\gamma \geqslant 2$ рассмотрим функцию

$$
g(\gamma)=\tau+\frac{2 \tau-2}{\tau^{2} \gamma-2}
$$

Очевидно, что $g(\gamma)$ строго убывает и что $g(2)=2, \lim _{\gamma \rightarrow+\infty} g(\gamma)=\tau$. Также для положительного Г определим величину

$$
C(\Gamma)=2^{18} \Gamma^{\left(\tau-\tau^{2}\right) /\left(\tau^{2} \gamma-2\right)} .
$$

ТеОрема 55. Пусть набор вещественных чисел $\theta^{1}, \theta^{2}$ является плохо приближаемым в следующем смысле. Для некоторых постоянных $\Gamma \in(0,1)$ и $\gamma \geqslant 2$ неравенство

$$
\left\|\theta^{1} m_{1}+\theta^{2} m_{2}\right\| \geqslant \frac{\Gamma}{\left(\max \left\{\left|m_{1}\right|,\left|m_{2}\right|\right\}\right)^{\gamma}}
$$

выполнено для всех иельх векторов $\left(m_{1}, m_{2}\right) \in \mathbb{Z}^{2} \backslash\{(0,0)\}$. Тогда найдется бесконечная последовательность целых двумерных векторов $\left(x_{1}(i), x_{2}(i)\right)$ такая, что:

1) $x_{1}(i), x_{2}(i)>0$;

2) $\left\|\theta^{1} x_{1}(i)+\theta^{2} x_{2}(i)\right\|\left(\max \left\{x_{1}(i), x_{2}(i)\right\}\right)^{g(\gamma)} \leqslant C(\Gamma)$ для всех $i$.

Мы не будем останавливаться на доказательстве этой теоремы. Оно развивает оригинальные идеи В. М. Шмидта и изложено в [91] достаточно подробно.

10.2. О линейных формах с числом переменных $k>2$. В случае $k \geqslant 3$ линейная форма от $k$ переменных, вообще говоря, может не принимать малые значения при положительных $x_{j}$. Приведем результат В. М. Шмидта из [87].

Теорема 56. Найдется набор чисел $\Theta=\left(\theta_{1}, \ldots, \theta_{k}\right)$ c $k \geqslant 3$ такой, что:

- выполнено равенство $\operatorname{dim}_{\mathbb{Z}} \Theta=k+1$;

- для любого положительного в найдется положительное с $(\varepsilon)$ такое, что

$$
\left\|\theta_{1} x_{1}+\cdots+\theta_{k} x_{k}\right\|>c(\varepsilon)\left(\max _{1 \leqslant i \leqslant k}\left|x_{i}\right|\right)^{-2-\varepsilon}
$$

для всех иелых $x_{1}, \ldots, x_{k}$ с условием $x_{i}>0, i=1, \ldots, k$. 
Для доказательства теоремы 56 используется результат Г. Давенпорта и В. М. Шмидта [92] о существовании чисел с аномальными совместными приближениями:

Теорема 57. Пусть $n \geqslant 2 u \psi(t)$ есть положительная функиия вещественного аргумента $t$. Тогда найдется набор чисел $\Theta=\left(\theta_{1}, \ldots, \theta_{n}\right)$ такой, чmo:

- выполнено равенство $\operatorname{dim}_{\mathbb{Z}} \Theta=n+1$;

- для любого достаточно болъшого $t$ найдется натуралъное $q \leqslant t$ такое, что неравенства

$$
\left\|q \theta_{j}\right\| \leqslant \psi(t)
$$

выполнены для всех значений индекса $j$ c $1 \leqslant j \leqslant n$, за возможным исключением одного значения $j_{0}=j_{0}(t)$.

Отметим, что при $n \geqslant 2$ и $\psi(t)=O\left(t^{-1}\right)$ при $t \rightarrow+\infty$ обеспечить выполнения неравенства (97) для всех $j$ из промежутка $1 \leqslant j \leqslant n$ нельзя; это противоречило бы теореме 17.

Следуя В. М. Шмидту [87], покажем, что из теоремы 57 с помощью простейших соображений переноса вытекает теорема 56. Для этого надо взять $n=k-1$ и $\psi(t)=e^{-t}$. Для чисел $\theta_{1}, \ldots, \theta_{k-1}$, существование которых обеспечивается теоремой 57 , найдем такое число $\theta_{k}$, для которого с некоторой положительной постоянной $c_{1}(\varepsilon)$

$$
\left\|\theta_{i} u+\theta_{k} v\right\|>c_{1}(\varepsilon)(|u|+|v|)^{-2-\varepsilon}, \quad 1 \leqslant i \leqslant k-1,
$$

для всех целых $u, v$ с условием $v \neq 0$. (Стандартные аргументы, связанные с применением леммы Бореля-Кантелли, показывают, что это неравенство выполнено для почти всех чисел $\theta_{k}$. Мы проводили подобные рассуждения в пп. 2.2 и 2.3 настоящей статьи.)

Теперь для целой точки $\left(x_{1}, \ldots, x_{k}\right)$ с достаточно большим значением $M=$ $\max _{1 \leqslant i \leqslant k}\left|x_{i}\right|$ полагаем $t=(\log M)^{2}$ и выбираем $q$ из интервала $1 \leqslant q \leqslant t$ удовлетворяющим заключению теоремы 57 . Не ограничивая общности, считаем, что $j_{0}(t)=1$. Значит, если $x_{k} \neq 0$, то

$$
\begin{aligned}
\left\|\theta_{1} x_{1}+\cdots+\theta_{k} x_{k}\right\| & \geqslant q^{-1}\left\|\theta_{1} q x_{1}+\cdots+\theta_{k} q x_{k}\right\| \\
& \geqslant q^{-1}\left(\left\|\theta_{1} q x_{1}+\theta_{k} q x_{k}\right\|-M\left(\left\|\theta_{2} q\right\|+\cdots+\left\|\theta_{k-1} q\right\|\right)\right) \\
& >q^{-1}\left(c_{1}\left(\frac{\varepsilon}{3}\right)(q M)^{-2-\varepsilon / 3}-k M e^{-t}\right)>M^{-2-\varepsilon},
\end{aligned}
$$

и теорема 56 доказана.

\section{1. Задача В. В. Козлова}

Рассмотрим набор вещественных чисел $\Theta=\left(\theta^{1}, \ldots, \theta^{m}\right)$, линейно независимых над $\mathbb{Z}$, и вещественнозначную функцию $f\left(x_{1}, \ldots, x_{m}\right)$, периодичную по каждому из аргументов $x_{i}$ с периодом 1 , о которой мы будем предполагать, что она достаточно гладкая (например, непрерывная). Всюду ниже в этом разделе 
будем предполагать, что выполняется условие нулевого среднего

$$
\int_{0}^{1} \cdots \int_{0}^{1} f\left(x_{1}, \ldots, x_{m}\right) d x_{1} \cdots d x_{m}=0 .
$$

Согласно знаменитой теореме Г. Вейля [93], для непрерывной функции $f$ и для любой начальной фазы $\mathbf{y}=\left(y_{1}, \ldots, y_{m}\right) \in \mathbb{R}^{m}$

$$
I(T, \mathbf{y})=I^{[f, \Theta]}(T, \mathbf{y})=\int_{0}^{T} f\left(\theta^{1} t+y_{1}, \ldots, \theta^{m} t+y_{m}\right) d t=o(T), \quad T \rightarrow+\infty .
$$

Нетривиальную задачу о возвращаемости и осцилляции интеграла $I(T, \mathbf{y})$ поставил В. В. Козлов.

Мы будем говорить, что интеграл $I(T, \mathbf{y})$ осииллирует (при заданной начальной фазе $\mathbf{y})$, если множества моментов времени

$$
\mathfrak{T}_{+}=\left\{T \in \mathbb{R}_{+} \mid I(T, \mathbf{y})>0\right\}, \quad \mathfrak{T}_{-}=\left\{T \in \mathbb{R}_{+} \mid I(T, \mathbf{y})<0\right\}
$$

оба являются неограниченными сверху.

Мы будем говорить, что интеграл $I(T, \mathbf{y})$ возвращается (при заданной начальной фазе $\mathbf{y})$, если

$$
\liminf _{T \rightarrow+\infty}|I(T, \mathbf{y})|=0 .
$$

У описанной выше непрерывной постановки задачи имеется дискретный аналог.

Рассмотрим набор вещественных чисел $\Theta=\left(\theta^{1}, \ldots, \theta^{m}\right)$, линейно независимых вместе с единицей над $\mathbb{Z}$, и (достаточно гладкую) вещественнозначную функцию $F\left(x_{1}, \ldots, x_{m}\right)$, периодичную по каждому из аргументов $x_{i}$ с периодом 1 и имеющую нулевое среднее:

$$
\int_{0}^{1} \cdots \int_{0}^{1} F\left(x_{1}, \ldots, x_{m}\right) d x_{1} \cdots d x_{m}=0 .
$$

Упомянутая выше теорема Г. Вейля о равенстве пространственного и временного средних в этом случае утверждает, что для любой начальной фазы $\mathbf{y}=\left(y_{1}, \ldots, y_{m}\right) \in \mathbb{R}^{m}$ выполнено

$$
S(Q, \mathbf{y})=S^{[F, \Theta]}(Q, \mathbf{y})=\sum_{s=1}^{Q} F\left(\theta^{1} s+y_{1}, \ldots, \theta^{m} s+y_{m}\right) d t=o(Q), \quad Q \rightarrow+\infty
$$

Осцилляция и возвращаемость суммы $S(Q, \mathbf{y})$ при $Q \rightarrow+\infty$ определяется, естественно, аналогично осцилляции и возвращаемости интеграла $I(T, \mathbf{y})$.

Если для функции $m$ переменных $f\left(x_{1}, \ldots, x_{m}\right)$ рассмотреть функцию $F_{z}\left(y_{1}, \ldots, y_{m-1}\right)$ от $m-1$ переменных $y_{1}, \ldots, y_{m-1}$ (переменная $z$ теперь рассматривается как параметр), определяемую соотношением

$$
F_{z}\left(y_{1}, \ldots, y_{m-1}\right)=\int_{0}^{1 / \theta^{m}} f\left(\theta^{1} t+y_{1}, \ldots, \theta^{m-1} k+y_{m-1}, \theta^{m} t+z\right) d t,
$$

и новый набор частот $\Theta^{*}=\left(\theta^{1} / \theta^{m}, \ldots, \theta^{m-1} / \theta^{m}\right)$ (состоящий из чисел, линейно независимых вместе с единищей над $\mathbb{Z}$ ), то из возвращаемости (или осцилляции) суммы $S^{\left[F_{z}, \Theta\right]}(Q, \mathbf{y})$ при некотором значении параметра $z$ и при некоторой 
начальной фазе $\mathbf{y}=\left(y_{1}, \ldots, y_{m-1}\right)$ будет следовать возвращаемость (или осцилляция) интеграла $I(T, \mathbf{x})$ при соответствующей начальной фазе $\mathbf{x}$. Заметим, что обратное утверждение, вообще говоря, может оказаться неверным.

11.1. Лемма Переса и теорема Халаса. В этом пункте мы приведем два общих утверждения из эргодической теории и обсудим, какие следствия они дают в рассматриваемой задаче.

Под динамической системой здесь мы понимаем вероятностное пространство $(\Omega, \mathscr{A}, \mu)$ с заданным сохраняющим меру $\mu$ эргодическим преобразованием $T$ множества $\Omega$ в себя (об основных понятиях эргодической теории см. [94]). Эргодическая теорема Биркгофа утверждает, что для любой интегрируемой функции $g$ из $\Omega$ в $\mathbb{R}$ для почти каждого $x \in \Omega$ выполнено

$$
\frac{1}{Q} \sum_{s=1}^{Q} g\left(T^{s} x\right) \rightarrow \int_{\Omega} g d \mu, \quad Q \rightarrow+\infty .
$$

Следующее утверждение известно как лемма Переса.

ЛЕмма 6 (Ю. Перес [95]). Пусть $\Omega$ компактно, а преобразование $T$ непрерывно. Тогда для любой непрерьвной функции $g$ из $\Omega$ в $\mathbb{R}$ найдется точка $x \in \Omega$ такая, что

$$
\frac{1}{Q} \sum_{s=1}^{Q} g\left(T^{s} x\right) \geqslant \int_{\Omega} g d \mu .
$$

В рассматриваемой нами задаче лемма 6 влечет

СлеДСТвИЕ. Для произвольного набора $\Theta$, состоящего из линейно независимых вместе с единицей над $\mathbb{Z}$ частот, и для любой непрерывной функции $F\left(x_{1}, \ldots, x_{m}\right)$, периодичной по каждому аргументу с периодом 1 и имеющей нулевое среднее, найдется точка $\mathbf{y} \in[0,1)^{m}$ такая, что

$$
S^{[F, \Theta]}(Q, \mathbf{y}) \geqslant 0 \quad \forall Q \in \mathbb{N} .
$$

Аналогичное утверждение для интеграла $I^{[f, \Theta]}(T, \mathbf{y})$ имеется у П. Боля [96]. В несколько более сильном виде оно имеется у В. В. Козлова [97], [98].

Теорема 58 (В. В. Козлов [97], [98]). (i) Пусть частоты $\theta^{1}, \ldots, \theta^{m}$ линейно независимы над $\mathbb{Z}$ и периодическая функиия $f$ непрерывна и имеет нулевое среднее. Тогда найдется точка $\mathbf{y} \in[0,1)^{m}$ такая, что

$$
I^{[f, \Theta]}(T, \mathbf{y}) \geqslant 0 \quad \forall T \in \mathbb{R}
$$

$u$, кроме того, $f(\mathbf{y})=0$.

(ii) Если частоты $\theta^{1}, \ldots, \theta^{m}$ линейно зависимы над $\mathbb{Z}$, то существуют, по крайней мере, две различные точки $\mathbf{y}_{1}, \mathbf{y}_{2} \in[0,1)^{m}$, для которых выполнено заключение пункта (i).

Сформулируем одну из теорем из работы Г. Халаса [99], касающейся поведения сумм Биркгофа для интегрируемой на $\Omega$ функции $g$. 
Теорема 59 (Г. Халас [99]). Для любой интегрируемой g разность

$$
\sum_{s=1}^{Q} g\left(T^{s} x\right)-Q \int_{\Omega} g d \mu
$$

бесконечно много раз меняет знак (в слабом смысле) для почти всех $x \in \Omega$.

То, что величина меняет знак в слабом смысле, в данном случае означает что, когда величина обращается в нуль, мы можем также считать ее поменявшей знак, т. е. теорема 59 утверждает, что величина (100) не может, начиная с какого-то момента, быть всегда строго положительной или строго отрицательной.

СлЕДСТвИЕ. Для произвольного набора $\Theta$, состоящего из линейно независимых вместе с единицей над $\mathbb{Z}$ частот, и для произвольной интегрируемой функиии $F\left(x_{1}, \ldots, x_{m}\right)$, периодичной по каждому аргументу с периодом 1 и имеющей нулевое среднее, величина $S^{[F, \Theta]}(Q, \mathbf{y})$ бесконечно много раз меняет знак (в слабом смысле) для почти всех значений началъных фаз у.

11.2. Индивидуальная возвращаемость. В случае $m=2$ возвращаемость интеграла $I^{[f, \Theta]}(T, \mathbf{y})$ для периодической функции $f\left(x_{1}, x_{2}\right) \in C^{2}\left([0,1]^{2}\right)$ с нулевым средним, произвольного набора частот $\Theta=\left(\theta^{1}, \theta^{2}\right), \theta^{1} / \theta^{2} \notin \mathbb{Q}$, и для произвольных начальных фаз $\left(y_{1}, y_{2}\right)$ была доказана самим В. В. Козловым в [97], [98]. В этих же работах В. В. Козлов заметил, что при $m=2$ имеет место более точное утверждение о возвращаемости интеграла $I(T, \mathbf{y})$, а именно, найдется последовательность положительных цельх чисел $T_{\nu}$ такая, что одновременно

$$
\max _{1 \leqslant i \leqslant 2}\left\|\theta^{i} T_{\nu}\right\| \rightarrow 0, \quad I\left(T_{\nu}, \mathbf{y}\right) \rightarrow 0, \quad \nu \rightarrow+\infty .
$$

Далее В. В. Козлов заметил, что из условия $f\left(y_{1}, y_{2}\right) \neq 0$ с помощью уже доказанной "сильной возвращаемости" (101) следует осцилляция интеграла $I(T, \mathbf{y})$ при рассмотренном значении начальной фазы $\mathbf{y}=\left(y_{1}, y_{2}\right)$.

Эти результаты В. В. Козлова были усилены Е. А. Сидоровым [100], о чем мы подробно поговорим в следующем пункте. В случае произвольного значения размерности $m$ результаты об осцилляции и возвращаемости были получены С. В. Конягиным в случае нечетной функции $f$ (см. [101]) и Н. Г. Мощевитиным в общем случае (см. [102], [103]). Ниже мы приведем формулировки этих результатов и дадим некоторые комментарии.

Сначала мы сформулируем две теоремы, доказанные в [102] и [103].

ТЕОРема 60. Предположим, что $m \geqslant 2$, функиия $f$ принадлежит классу $C^{w}\left([0,1]^{m}\right)$, где

$$
w=w(m)=[\exp (20 m \log m)]
$$

и что выполнено условие нулевого среднего (98). Тогда для любых частот $\Theta=\left(\theta^{1}, \ldots, \theta^{m}\right)$, линейно независимьх над $\mathbb{Z}$, при любой начальной фазе $\mathbf{y}$ интеграл $I(T, \mathbf{y})$ возвращается. 
Теорема 61. Если в условиях теоремы 60 дополнительно потребовать, чтобы для начальной фазы $\mathbf{y}=\left(y_{1}, \ldots, y_{m}\right)$ выполнялось

$$
f\left(y_{1}, \ldots, y_{m}\right) \neq 0
$$

то интеграл $I(T, \mathbf{y})$ будет осииллировать.

Доказательства теоремы 60 можно найти в работах автора [102] и [103]. Однако там она сформулирована с худшим значением показателя гладкости $w(m)$. Тем не менее, доказательство из [102], [103] дословно проходит для показателя (102). К сожалению, соответствующая выкладка проведена только в диссертации автора [104]. Тем не менее, она легко восстанавливается. Теорема 61, вообще говоря, не является непосредственным следствием теоремы 60, но ее доказательство совершенно аналогично доказательству теоремы 60. Точное рассуждение тоже приведено в диссертации автора [104].

Из теоремы 60 очевидным образом получаем следующее утверждение.

СледствиЕ. Пусть бункиия $F(\mathbf{x})$ принадлежит классу $C^{w(m)}\left([0,1]^{m}\right) u$ имеет нулевое среднее (99). Тогда для любых частот $\Theta=\left(\theta^{1}, \ldots, \theta^{m}\right)$, линейно независимых вместе с единищей над $\mathbb{Z}$, и для всякой начальной фазы $\mathbf{x}$ имеет место неравенство

$$
\liminf _{Q \rightarrow+\infty}|S(Q, \mathbf{x})|<+\infty .
$$

Понятно, что следствие не обеспечивает возвращаемости суммы $S(Q, \mathbf{y})$. По-видимому, вопрос о возвращаемости суммы $S(Q, \mathbf{y})$ остается открытым.

Отметим также, что доказательства теорем 60 и 61 связаны и с исследованием распределений наилучших диофантовых приближений линейной формы (случай $n=1)$, и с тонким анализом осцилляции гармоник в разложении функции $f$ в кратный ряд Фурье.

Сформулируем теперь результат С. В. Конягина из [101].

ТЕОРема 62. Предположим, что $m \geqslant 3$, функиия $f$ принадлежит классу $C^{w_{1}}\left([0,1]^{m}\right)$, гдe

$$
w_{1}=w_{1}(m)=3 m \cdot 2^{m-1},
$$

и что выполнено условие нулевого среднего (98). Дополнительно предположим, что для всех $\mathbf{x}$

$$
f\left(-x_{1}, \ldots,-x_{m}\right)=-f\left(x_{1}, \ldots, x_{m}\right) .
$$

Тогда интеграл $I(T, \mathbf{0})$ возвращается.

Отметим, что $w_{1}(m)<w(m)$ и, следовательно, в теореме С. В. Конягина о нечетной функции условие на гладкость $f$ является более слабым, чем в теореме 60 .

Заметим, что теорема 62 может быть обобщена следующим образом: если рассмотреть не одну функцию $f$, удовлетворяющую условию теоремы 62 , а конечныци набор функций $f_{1}, \ldots, f_{r}$, каждая из которых удовлетворяет условию теоремы 62 , то

$$
\liminf _{T \rightarrow+\infty} \sum_{1 \leqslant j \leqslant r}\left|I^{\left[f_{j}, \Theta\right]}(T, \mathbf{0})\right|=0 .
$$


Доказательство из [101] обобщается непосредственно. Сделаем несколько замечаний о том, какой гладкости функции $f$ недостаточно для возвращаемости. Н. Г. Мощевитин (см. [105]), основываясь на идеях Д. В. Трещёва, обобщил пример А. Пуанкаре из [106], [107] и доказал следующее утверждение.

Теорема 63. Пусть набор частот $\Theta=\left(\theta^{1}, \ldots, \theta^{m}\right)$ является плохо приближаемым, т.е. с некоторой положительной постоянной $\Gamma=\Gamma(\Theta)$ для всех ненулевых иелых векторов $\mathbf{k}=\left(k_{1}, \ldots, k_{m}\right) \in \mathbb{Z}^{m}$ выполняется неравенство

$$
\left|k_{1} \theta^{1}+\cdots+k_{m} \theta^{m}\right|>\Gamma \cdot\left(\max _{1 \leqslant i \leqslant m}\left|k_{i}\right|\right)^{1-m} .
$$

Тогда найдется 1-периодическая по каждой переменной функиия $f^{[\Theta]}(\mathbf{x})$ класса $C^{m-2}\left([0,1]^{m}\right) \backslash C^{m-1}\left([0,1]^{m}\right)$ имеющая нулевое среднее и такая, что

$$
\lim _{T \rightarrow+\infty} I^{\left[f^{[\Theta]}, \Theta\right]}(T, \mathbf{0})=+\infty .
$$

Отметим, что для плохо приближаемого набора условие линейной независимости над $\mathbb{Z}$ выполнено автоматически.

C другой стороны, построив некоторый сингулярный набор частот $\Theta$, С. В. Конягин в [101] получил следующий результат.

Теорема 64. При $m \geqslant 4$ положим

$$
w_{3}=w_{3}(m)=\left[\frac{2^{m-1}(m-2)^{m-2}}{(m-1)^{m-1}}\right]-1 .
$$

Тогда найдутся периодическая функиия $f \in C^{w_{3}}\left([0,1]^{m}\right)$ и набор частот $\Theta=$ $\left(\theta^{1}, \ldots, \theta^{m}\right)$, состоящий из линейно независимых над $\mathbb{Z}$ чисел такие, что въполнено (104) и при этом

$$
\lim _{T \rightarrow+\infty} I^{\left[f^{[\Theta]}, \Theta\right]}(T, \mathbf{0})=+\infty .
$$

Заметим, что при $m \geqslant 9$ теорема 64 дает пример более гладкой функции $f$ (чем теорема 63), для которой нет возвращаемости интеграла $I^{\left[f^{[\Theta]}, \Theta\right]}(T, \mathbf{0})$.

Отрицательный результат об отсутствии одновременной возвращаемости в общем случае имеется в [102]. Он тоже связан с использованием сингулярных наборов $\Theta$. Приведем его формулировку.

ТеОРема 65. Пусть $\Phi(t)$ - сколь угодно быстро убывающая к нулю функиия. Тогда найдутся две вещественнозначные функиии

$$
f_{j}\left(x_{1}, x_{2}, x_{3}\right)=\sum_{\left(k_{1}, k_{2}, k_{3}\right) \in \mathbb{Z}^{3} \backslash\{\mathbf{0}\}} f_{j ; k_{1}, k_{2}, k_{3}} \exp \left(2 \pi i\left(k_{1} x_{1}+k_{2} x_{2}+k_{3} x_{3}\right)\right),
$$

$j=1,2$, такие, что их коэффициенты Фуръе допускают оценку

$$
\left|f_{j ; k_{1}, k_{2}, k_{3}}\right| \leqslant \Phi\left(\max _{1 \leqslant j \leqslant 3}\left|k_{j}\right|\right) \quad \forall k \in \mathbb{Z}^{3}, \quad j=1,2,
$$

но для некоторого набора $\Theta=\left(\theta^{1}, \theta^{2}, \theta^{3}\right)$, состоящего из трех чисел, линейно независимых над $\mathbb{Z}$ выполняется

$$
\liminf _{T \rightarrow+\infty} \sum_{1 \leqslant j \leqslant 2}\left|I^{\left[f_{j}, \Theta\right]}(T, \mathbf{0})\right|=+\infty .
$$


Отметим также работу автора [108], в которой имелось частичное решение проблемы при $m=3$.

11.3. Равномерная возвращаемость. Когда мы говорим о равномерной возвращаемости, то имеем в виду, что возвращаются величины

$$
J(T)=J^{[f, \Theta]}(T)=\sup _{\mathbf{y} \in \mathbb{R}^{m}}\left|I^{[f, \Theta]}(T, \mathbf{y})\right|, \quad R(Q)=R^{[f, \Theta]}(Q)=\sup _{\mathbf{y} \in \mathbb{R}^{m}}\left|S^{[F, \Theta]}(Q, \mathbf{y})\right| .
$$

Результат Е. А. Сидорова, упоминавшийся выше, относится именно к равномерной возвращаемости. Сейчас мы его сформулируем.

Теорема 66 (Е.А. Сидоров [100]). Пусть $m=1$ и функиия $F(x)$ является 1-периодической абсолютно непрерывной функцией вещественной переменной $x$. Пусть $\theta \notin \mathbb{Q}$. Тогда имеет место равномерная возвращаемость суммы $S^{[F, \Theta]}(Q, y)$, m.e.

$$
\liminf _{Q \rightarrow+\infty} R(Q)=0 .
$$

Доказательство Е. А. Сидорова использует аппарат цепных дробей.

Как обнаружено в [109], случай $m>1$ существенно отличается от случая $m=1$. В работе [109] автор доказал отсутствие равномерной возвращаемости уже при $m=2$ и даже для гладкой $F\left(x_{1}, x_{2}\right)$. Для этого он использовал сингулярный набор $\left(\theta_{1}, \theta_{2}\right)$, передоказав теорему А. Я. Хинчина 1926 г. (теорему 1 ), о которой он в то время не знал. В настоящем обзоре мы приводим формулировку более общего результата Е. В. Коломейкиной и Н. Г. Мощевитина [110] и основного вспомогательного утверждения (о существовании сингулярных наборов $\Theta$ специального вида при $n=1)$.

Рассмотрим разложение в ряд Фурье

$$
F\left(x_{1}, \ldots, x_{m}\right)=\sum_{\left(k_{1}, \ldots, k_{m}\right) \in \mathbb{Z}^{m} \backslash\{\mathbf{0}\}} F_{k_{1}, \ldots, k_{m}} \exp \left(2 \pi i\left(k_{1} x_{1}+\cdots+k_{m} x_{m}\right)\right) .
$$

Cпектром функции $F$ будем называть множество

$$
\operatorname{spec} F=\left\{\left(k_{1}, \ldots, k_{m}\right) \in \mathbb{Z}^{m}: F_{k_{1}, \ldots, k_{m}} \neq 0\right\} .
$$

Следующий результат показывает, что равномерной возвращаемости может не быть даже в случае очень большой гладкости функции $F$.

ТЕОРема 67. Пусть периодическая функиия $F$ с периодом 1 принадлежит классу $C^{1}\left([0,1]^{m}\right)$ и выполнено (99).

Рассмотрим следующие два условия:

(A) для любой положительнозначной функции $\lambda(t)=o(t), t \rightarrow+\infty$, найдется набор чисел $\Theta=\left(\theta^{1}, \ldots, \theta^{m}\right)$, линейно независимых вместе с единицей над $\mathbb{Z}$, такой, что

$$
R^{[F, \Theta]}(Q)>\lambda(Q)
$$

при всех достаточно больиих $Q$;

(B) найдутся положительное $R$ и ненулевая целая точка $\left(p_{1}, \ldots, p_{m}\right) \in \mathbb{Z}^{m}$ такие, что $\operatorname{spec} F \subset \mathscr{B}(R) \cup \mathscr{L}(p)$, где $\mathscr{B}(R)$ обозначает иар в $\mathbb{R}^{m}$ с иентром в нуле и радиусом $R$, а $\mathscr{L}(p)$ обозначает прямую в $\mathbb{R}^{m}$, проходящую через начало координат и точку $p$.

Тогда условие (A) эквивалентно отрицанию условия (B). 
Для доказательства теоремы 67 используется следующая лемма.

ЛЕмма 7. В предположении отрищания условия (В) теоремы 67 для произвольной функции $\psi(t)$, монотонно убъвающей $\kappa$ нулю при $t \rightarrow+\infty$, найдутся набор $\Theta=\left(\theta^{1}, \ldots, \theta^{m}\right)$, состоящий из чисел, линейно независимых вместе с единицей над $\mathbb{Z}$, и бесконечная последовательность векторов

$$
\left(k_{1, \nu}, \ldots, k_{m, \nu}\right) \in \operatorname{spec} F, \quad \nu=1,2,3, \ldots,
$$

такие, что для каждого $\nu$

$$
\left\|\theta^{1} k_{1, \nu}+\cdots+\theta^{m} k_{m, \nu}\right\| \leqslant \psi\left(\max _{1 \leqslant i \leqslant m}\left|k_{i, \nu+1}\right|\right) .
$$

Понятно, что условие (105) очень похоже на соотношение (19), фигурирующее в эквивалентном определении $\psi$-сингулярности (в лемме 7 речь идет как бы о "наилучших приближениях из множества $\operatorname{spec} F$ ").

Отметим, что если набор $\Theta$ не является $\psi$-сингулярным (при некоторой функции $\psi$ ), то получить утверждение о равномерной возвращаемости можно очень легко: имеет место следующее утверждение.

ПРеДЛОЖЕНИЕ 4. Рассмотрим убъвающую $\kappa$ нулю при $t \rightarrow+\infty$ функиию $\Phi(t)$ и предположим, что ряд

$$
\sum_{k_{1}, \ldots, k_{m}=-\infty}^{+\infty} \Phi\left(\max _{1 \leqslant i \leqslant m}\left|k_{i}\right|\right) \max _{1 \leqslant i \leqslant m}\left|k_{i}\right|
$$

сходится. Предположим, что коэффициенть Фуръе функции $F\left(x_{1}, \ldots, x_{m}\right)$ допускают оченку

$$
\left|F_{k_{1}, \ldots, k_{m}}\right| \leqslant \Phi\left(\max _{1 \leqslant i \leqslant m}\left|k_{i}\right|\right)
$$

и что выполнено условие нулевого среднего (99). Рассмотрим (стремяшуюся $\kappa$ нулю) функиию

$$
\Phi_{1}(t)=\sum_{\left(k_{1}, \ldots, k_{m}\right): \max _{1 \leqslant i \leqslant m}\left|k_{i}\right| \geqslant t} \Phi\left(\max _{1 \leqslant i \leqslant m}\left|k_{i}\right|\right) .
$$

Пусть $n=1$ и набор $\Theta=\left(\theta^{1}, \ldots, \theta^{m}\right)$, состоящий из линейно независимьх вместе с единицей над $\mathbb{Z}$ вещественных чисел, не является $\psi$-сингулярным ни при какой функции $\psi(t)$ такой, что

$$
\psi(t)\left(\Phi_{1}(t)\right)^{-1 / m} \rightarrow+\infty, \quad t \rightarrow+\infty .
$$

Тогда найдется последовательность цельх чисел $q_{\mu}, \mu=1,2,3, \ldots$, такая, что

$$
R^{[F, \Theta]}\left(q_{\mu}\right) \rightarrow 0, \quad \max _{1 \leqslant i \leqslant m}\left\|\theta^{i} q_{\mu}\right\| \rightarrow 0, \quad \mu \rightarrow+\infty .
$$

Сделаем пояснение. Например, условия предложения будут выполнены, если от коэффициентов Фурье функции $F$ потребовать выполнения неравенства

$$
\left|F_{k_{1}, \ldots, k_{m}}\right| \leqslant \Gamma\left(\max _{1 \leqslant i \leqslant m}\left|k_{i}\right|\right)^{-\gamma} \forall \mathbf{k} \in \mathbb{Z}^{m} \backslash\{\mathbf{0}\}
$$


с некоторыми $\Gamma>0, \gamma>(m+1) m$, а от набора $\Theta$ потребовать выполнения соотношения

$$
\limsup _{t \rightarrow+\infty} \psi_{\Theta}(t) t^{\gamma / m-1}=+\infty
$$

Понятно, что предложение 4 утверждает существование "сильной возвращаемости", когда выполнены одновременно оба соотношения из (101), причем эта возвращаемость носит равномерный характер. Из доказательства очевидно, что аналог предложения 4 справедлив и для одновременной возвращаемости интегралов нескольких функций. В свете предложения 4 становится ясно, что основную трудность в доказательстве теорем 60 и 61 составляет разбор случая, когда набор $\Theta$ является $\psi$-сингулярным с очень быстро убывающей функцией $\psi$.

ДОКАЗАТЕЛЬСТВО ПРЕДЛОЖЕНИЯ 4 . Невыполнение условия $\psi$-сингулярности, согласно предложению 1 , означает, что для некоторой бесконечной подпоследовательности $\nu_{\mu}$ наилучших приближений

$$
\zeta_{\nu_{\mu}} \cdot\left(\Phi_{1}\left(M_{\nu_{\mu}+1}\right)\right)^{-1 / m} \rightarrow+\infty, \quad \mu \rightarrow+\infty .
$$

Понятно, что

$$
\lambda_{\nu_{\mu}}=\zeta_{\nu_{\mu}} \cdot \Phi_{1}\left(M_{\nu_{\mu}+1}\right) \rightarrow 0, \quad \mu \rightarrow+\infty
$$

Положим

$$
Q_{\mu}=\left(\zeta_{\nu_{\mu}} \cdot \Phi_{1}\left(M_{\nu_{\mu}+1}\right)\right)^{-m /(m+1)}
$$

и определим по теореме Дирихле такое $q_{\mu}$, что

$$
1 \leqslant q_{\mu} \leqslant Q_{\mu}, \quad \max _{1 \leqslant i \leqslant m}\left\|\theta_{i} q_{\mu}\right\| \leqslant Q_{\mu}^{-1 / m} .
$$

При этом

$$
q_{\mu} \rightarrow+\infty, \quad \max _{1 \leqslant i \leqslant m}\left\|\theta_{i} q_{\mu}\right\| \rightarrow 0, \quad \mu \rightarrow+\infty .
$$

С другой стороны,

$$
\begin{aligned}
& R^{[F, \Theta]}\left(q_{\mu}\right) \leqslant \sum_{\mathbf{k} \in \mathbb{Z}^{m} \backslash\{\mathbf{0}\}}\left|F_{k_{1}, \ldots, k_{m}}\right|\left|\sum_{s=1}^{q_{\mu}} \exp \left(2 \pi i\left(k_{1} \theta^{1}+\cdots+k_{m} \theta^{m}\right) s\right)\right| \\
& \ll \sum_{\mathbf{k}: \max _{i}\left|k_{i}\right|<M_{\nu_{\mu}+1}} \Phi\left(\max _{i}\left|k_{i}\right|\right) \frac{\max _{i}\left|k_{i}\right| \cdot \max _{i}\left\|\theta_{i} q_{\mu}\right\|}{\left\|k_{1} \theta^{1}+\cdots+k_{m} \theta^{m}\right\|}+q_{\mu} \Phi_{1}\left(M_{\nu_{\mu}+1}\right) \\
& \ll \frac{1}{\zeta_{\nu_{\mu}} Q_{\mu}^{1 / m}}+Q_{\mu} \Phi_{1}\left(M_{\nu_{\mu}+1}\right)=2\left(\zeta_{\nu_{\mu}}^{m}\left(\Phi_{1}\left(M_{\nu_{\mu}+1}\right)\right)^{-1}\right)^{-1 /(m+1)} \rightarrow 0, \\
& \mu \rightarrow+\infty .
\end{aligned}
$$

В последнем неравенстве использовано (106). Предложение доказано. 


\section{2. Сингулярные системы специального вида}

Некоторые утверждения из предыдущих разделов нашей статьи представляют собой теоремы существования сингулярных матриц, обладающих специальными свойствами. К таковым относятся, например, теоремы 6, 34, 12, 13, 41 и лемма 7.

В настоящем разделе мы приведем еще два утверждения о существовании сингулярных систем со специальными свойствами.

Первому утверждению следует предпослать два замечания. Во-первых, если рассмотреть случай $n=1$ и $m=2$, то (как отмечалось в разделе 2 и п. 5.3) бесконечно много раз будут встречаться тройки подряд идущих независимых наилучших приближений и, таким образом, для бесконечно многих значений $\nu$ будет выполнено

$$
\zeta_{\nu} \geqslant \frac{1}{6 M_{\nu+1} M_{\nu+2}} .
$$

Во-вторых, хотим отметить, что для сколь угодно быстро убывающей к нулю при $t \rightarrow+\infty$ функции $\psi(t)$ при $n=1$ и любом $m \geqslant 2$ автором в [35] было анонсировано (относительно подробное доказательство есть в [33]) существование векторов $\Theta \in \mathbb{R}^{m}$ таких, что $\operatorname{dim}_{\mathbb{Z}} \Theta=m+1$ и

$$
\zeta_{\nu} \leqslant \psi\left(M_{\nu+m-1}\right)
$$

Последнее утверждение допускает существенное усиление.

ТЕОРема 68. Пусть $m \geqslant 3$ и задана сколь угодно быстро убывающая $к$ нулю при $t \rightarrow+\infty$ функция $\psi(t)$. Пусть также задана сколь угодно бъстро растущая к бесконечности последовательность натуральных чисел $\tau(\nu)$, $\nu=1,2,3, \ldots$. Тогда существуют $\Theta \in \mathbb{R}^{m}$ такие, что $\operatorname{dim}_{\mathbb{Z}} \Theta=m+1 u$

$$
\zeta_{\nu} \leqslant \psi\left(M_{\nu+\tau(\nu)}\right)
$$

Мы поясним доказательство этой теоремы в случае $m=3$.

Для векторов $\mathbf{w}, \mathbf{e} \in \mathbb{R}^{3}$ (предполагаем, что е есть вектор единичной длины) и положительных $\eta, \delta$ определим шаровой сектор

$$
B_{\eta, \delta}(\mathbf{w}, \mathbf{e})=\left\{\Theta:|\Theta-\mathbf{w}| \leqslant \eta,\left|\frac{\Theta-\mathbf{w}}{|\Theta-\mathbf{w}|}-\mathbf{e}\right| \leqslant \delta\right\}
$$

$(|\cdot|$ в данном случае обозначает евклидову норму, хотя, вообще говоря, специфика нормы не имеет значения). Для вектора $\mathbf{w}=\left(w^{1}, w^{2}, w^{3}\right) \in \mathbb{R}^{3}$ будем обозначать $\overline{\mathbf{w}}=\left(w^{1}, w^{2}, w^{3}, 1\right) \in \mathbb{R}^{4}$. Будем отождествлять пространство $\mathbb{R}^{3}$ с аффинным подпространством $\{(\mathbf{x}, y): y=1\} \subset \mathbb{R}^{4}$. Для доказательства теоремы 68 используется следующая лемма.

Лемма 8. Пусть имеются трехмерное линейное вполне рациональное подпространство $\Pi \subset \mathbb{R}^{4}, \operatorname{dim} \Pi=3$, с нормалью $\overline{\mathbf{w}}$ и два линейных подпространства $\pi_{1}, \pi_{2} \subset \Pi, \operatorname{dim} \pi_{1}=\operatorname{dim} \pi_{2}=2$. Пусть также заданъ натуральные числа $a<b<$ с и векторь

$$
\mathbf{z}_{\nu}=\left(\mathbf{x}_{\nu}, y_{\nu}\right)=\left(x_{1, \nu}, x_{2, \nu}, x_{3, \nu}, y_{\nu}\right) \in \mathbb{Z}^{4}, \quad 1 \leqslant \nu \leqslant c
$$


такие, что:

(i) $\mathbf{z}_{a}, \mathbf{z}_{a+1}, \ldots, \mathbf{z}_{b-1}, \mathbf{z}_{b} \in \pi_{1}$;

(ii) $\mathbf{z}_{b}, \mathbf{z}_{b+1}, \ldots, \mathbf{z}_{c-1}, \mathbf{z}_{c} \in \pi_{2}$;

(iii) $\mathbf{z}_{b-1}, \mathbf{z}_{b}, \mathbf{z}_{b+1}$ линейно независимы.

Пусть имеются положительные числа $\eta, \delta$ и вектор $\mathbf{e} \in \mathbb{R}^{3}$ такие, что выполнено следующее: вектор е ортогонален подпространству $\pi_{2}$ и для любого $\Theta \in B_{\eta, \delta}(\mathbf{w}, \mathbf{e})$, с условием $\operatorname{dim}_{\mathbb{Z}} \Theta=4$, первые с векторов наилучших приближений суть именно векторы $\mathbf{z}_{\nu}, 1 \leqslant \nu \leqslant c$.

Пусть также задано натуральное $d>c$.

Тогда найдутся вполне рациональное линейное подпространство $\Pi_{*}$, $\operatorname{dim} \Pi_{*}=3$, с нормалью $\overline{\mathbf{w}}_{*}$, подпространство $\pi_{3} \subset \Pi_{*}, \operatorname{dim} \pi_{3}=2$, и последовательность иелочисленных векторов

$$
\mathbf{z}_{c+1}, \ldots, \mathbf{z}_{d-1}, \mathbf{z}_{d} \in \pi_{3}
$$

такие, что $\mathbf{z}_{c-1}, \mathbf{z}_{c}, \mathbf{z}_{c+1}$ линейно независимы, и для некоторых положительных $\eta_{*}, \delta_{*}$, а также некоторого вектора $\mathbf{e}_{*} \in \mathbb{R}^{3}$ выполнено следующее: вектор $\mathbf{e}_{*}$ ортогонален подпространству $\pi_{3}$ и для любого $\Theta \in B_{\eta_{*}, \delta_{*}}\left(\mathbf{w}_{*}, \mathbf{e}_{*}\right) \subset$ $B_{\eta, \delta}(\mathbf{w}, \mathbf{e})$ с условием $\operatorname{dim}_{\mathbb{Z}} \Theta=4$ первые d векторов наилучших приближений суть именно векторы $\mathbf{z}_{\nu}, 1 \leqslant \nu \leqslant d$.

Изложим схему доказательства леммы 8. Целочисленная решетка $\mathbb{Z}^{4}$ распадается на трехмерные слои $\Pi_{r}$, параллельные подпространству П:

$$
\mathbb{Z}^{4}=\bigcup_{r \in \mathbb{Z}} \Pi_{r} .
$$

Сначала выбираем целую точку $\mathbf{z}_{c+1}$ из слоя $\Pi_{1}$ или $\Pi_{-1}$, “соседнего" к слою $\Pi_{0}=\Pi$ (какой именно надо выбирать соседний слой, определяется направлением вектора е). Можем считать, что абсолютная величина

$$
M_{c+1}=\max _{i=1,2,3}\left|x_{i, c+1}\right|
$$

очень велика по сравнению со всеми параметрами, фигурирующими в индуктивной предпосылке. Далее обозначаем

$$
\Pi_{*}=\operatorname{span}\left(\mathbf{z}_{c-1}, \mathbf{z}_{c}, \mathbf{z}_{c+1}\right), \quad \pi_{3}=\operatorname{span}\left(\mathbf{z}_{c}, \mathbf{z}_{c+1}\right),
$$

и пусть $\overline{\mathbf{W}}_{*}-$ нормаль к $\Pi_{*}$.

Векторы $\mathbf{z}_{c}, \mathbf{z}_{c+1}$ лежат в двумерном вполне рациональном подпространстве $\pi_{3}$. Мы можем найти последовательность векторов $\mathbf{z}_{c+2}, \ldots, \mathbf{z}_{d} \in \pi_{3} \cap \mathbb{Z}^{4}$ таких, чтобы они составляли последовательность всех наилучших приближений (бо́льших по норме, чем $\left|\mathbf{z}_{c+1}\right|_{\bullet}$ ) для последнего вектора $\mathbf{z}_{d}$ относительно решетки $\pi_{3} \cap \mathbb{Z}^{4}$ с индуцированной нормой. Так как $\Pi_{*} \supset \pi_{3} \ni \mathbf{z}_{c}, \ldots, \mathbf{z}_{d}$, то за счет выбора малых $\eta_{*}$ и $\delta_{*}$ (в зависимости от величин $\rho\left(\Pi_{*}\right)>0$ и $M_{d}=$ $\left.\max _{i=1,2,3}\left|x_{i, d}\right|\right)$ мы добиваемся выполнения утверждения леммы 8. При этом направление вектора $\mathbf{e}_{*}$ выбирается так, чтобы именно векторы $\mathbf{z}_{d-1}$ и $\mathbf{z}_{d}$ (а не $\mathbf{z}_{d}-\mathbf{z}_{d-1}$ и $\mathbf{z}_{d}$ ) были последними наилучшими приближениями из первых $d$ приближений для любого $\Theta$ из строящейся окрестности. (Подобная конструкция имеется у О. Н. Германа в [111], [112].) 
Для доказательства теоремы 68 лемма 8 применяется следующим образом. Сначала занумеровываются все трехмерные вполне рациональные подпространства в $\mathbb{R}^{4}$. Затем запускается индуктивный процесс построения вложенных окрестностей вида (107). Определяется последовательность индексов $\nu_{k}$ так, чтобы выполнялись равенства $\nu_{k+1}=\nu_{k}+\tau\left(\nu_{k}\right)$. Каждый раз лемма 8 применяется для $a=\nu_{k-1}, b=\nu_{k}, c=\nu_{k+1}$. При этом дополнительно требуется выбирать величину $\eta_{*}$ настолько малой, чтобы было выполнено неравенство

$$
\eta_{*}<\frac{\psi\left(M_{d}\right)}{8 M_{b}} .
$$

Тогда при $b \leqslant \nu \leqslant c$ получаем $\nu+\tau(\nu) \leqslant c+\tau(c)=d$ и

$$
\zeta_{\nu} \leqslant \zeta_{b}<\psi\left(M_{d}\right) \leqslant \psi\left(M_{\nu+\tau(\nu)}\right) .
$$

Кроме того, на каждом шаге следует отделяться от вполне рационального трехмерного подпространства, номер которого совпадает с номером шага.

Пользуясь случаем, приведем формулировку одного утверждения, анонсированного в [34].

ТеОрема 69. Пусть $n=1$ u $m \geqslant 2$. Тогда для произвольной сколь угодно бъстро убъвающей $\kappa$ нулю при $t \rightarrow+\infty$ функиии $\psi(t)$ найдется $\psi$-сингулярнъй набор $\Theta \in \mathbb{R}^{m}$ такой, что $\operatorname{dim}_{\mathbb{Z}} \Theta=m+1$ и для любого $\nu$ векторъ $\mathbf{z}_{\nu}, \mathbf{z}_{\nu+1}, \ldots, \mathbf{z}_{\nu+m}$ будут линейно независимы.

\section{3. Добавление}

13.1. О лакунарных последовательностях. Последовательность $\left\{t_{j}\right\}$, $j=1,2,3, \ldots$, положительных вещественных чисел называется лакунарной, если для некоторого $M>0$ выполнены неравенства

$$
\frac{t_{j+1}}{t_{j}} \geqslant 1+\frac{1}{M} \quad \forall j \in \mathbb{N} .
$$

А. Я. Хинчин в 1926 г. доказал, что для любой последовательности, удовлетворяющей (108), найдутся вещественное число $\alpha$ и положительное $\gamma$ такие, что

$$
\left\|t_{n} \alpha\right\| \geqslant \gamma \quad \forall n \in \mathbb{N} .
$$

Этот результат опубликован в работе [1] (Hilfssatz III), которую мы цитировали неоднократно. Его можно также найти в недавнем переиздании трудов А. Я. Хинчина [16] (работа [1], переведенная на русский язык, вошла в книгу [16]). Лемма 2 из п. 6.3 (с. 85) настоящей работы, естественно, являлась обобщением этого одномерного результата А. Я. Хинчина.

Отметим, что конструкция А.Я. Хинчина позволяет установить существование вещественного $\alpha$ и положительной абсолютной постоянной $\gamma$ таких, что

$$
\left\|t_{n} \alpha\right\| \geqslant \frac{\gamma}{(M \log M)^{2}} \quad \forall n \in \mathbb{N} .
$$

Полвека спустя, в 1975 г. П. Эрдёш [113] высказал гипотезу, что для произвольной лакунарной последовательности найдется вещественное число $\alpha$ такое, что 
дробные доли $\left\{\alpha t_{j}\right\}, j \in \mathbb{N}$ не будут всюду плотны в $[0,1]$. Ответ на вопрос П. Эрдёша, естественно, следовал из процитированной выше леммы А. Я. Хинчина. Однако этот результат А.Я. Хинчина был забыт. Решение гипотезы П. Эрдёша было опубликовано А. Поллингтоном [114] и Б. де Матаном [115]. Позже количественные уточнения имелись у И. Кацнельсона [116], Р. К. Ахунжанова и Н. Г. Мощевитина [117], А. Дубицкаса [118]. Наилучший известный количественный результат принадлежит Ю. Пересу и В. Шлагу [19]. Они доказали, что с некоторой абсолютной положительной постоянной $\gamma>0$ для каждой рассматриваемой последовательности $\left\{t_{j}\right\}$ найдется вещественное число $\alpha$ такое, что

$$
\left\|\alpha t_{j}\right\| \geqslant \frac{\gamma}{M \log M} \quad \forall j \in \mathbb{N} .
$$

Ю. Перес и В. Шлаг использовали весьма оригинальную конструкцию, связанную с применением специального варианта локальной леммы Ловаса.

После работы Ю. Переса и В. Шлага появился ряд статей, в которых предложенный метод применялся в различных диофантовых задачах (см. [119]-[126]). Отметим, что в задаче о лакунарной последовательности наилучшее значение постоянной $\gamma$ из (109), по-видимому, получается из работы И. П. Рочева [125].

Предложенный Ю. Пересом и В. Шлагом в работе [19] подход к построению плохо приближаемых (по отношению к лакунарной последовательности) вещественных чисел оказался применим во многих задачах теории диофантовых приближений. Ниже мы перечислим некоторые из них. Но прежде сделаем ряд замечаний метрического характера.

13.2. О некоторых метрических результатах. Во-первых, рассмотрим одномерную задачу о приближениях. Процитируем результат Дж. В. С. Касселса из работы [127]. Напомним, что возрастающая последовательность $\left\{t_{n}\right\}_{n=1}^{\infty}$ целых чисел называется $\Sigma$-последовательностью, если имеет место соотношение

$$
\liminf _{N \rightarrow \infty} \frac{1}{N} \sum_{n \leqslant N} \frac{\mu_{n}}{t_{n}}>0
$$

где $\mu_{n}$ обозначает количество дробей вида $j / t_{n}, 0<j<t_{n}$, не представимых в виде $i / t_{q}$ с натуральным $i$ и $q<n$. Примерами $\Sigma$-последовательностей могут служить произвольная лакунарная последовательность натуральных чисел, последовательность чисел вида $n^{d}, n=1,2,3, \ldots$, при фиксированном натуральном $d$ или последовательность Фюрстенберга (о которой идет речь ниже).

Теорема Дж.В.С. Касселса [127] утверждает, что если $\left\{t_{n}\right\}_{n=1}^{\infty}$ является $\Sigma$-последовательностью и функция $\psi(n)$ монотонно убывает к нулю при $n$ стремящемся к бесконечности, то в случае расходимости ряда $\sum_{n=1}^{\infty} \psi(n)$ для почти
всех $\xi$ выполнено

$$
\liminf _{n \rightarrow+\infty}\left\|t_{n} \xi\right\|(\psi(n))^{-1}=0 .
$$

Во-вторых, сформулируем случай расходимости из теоремы А. Я. Хинчина [3] (в задаче о совместных приближениях и в том виде, в котором он присутствует в главе III книги [24]). Пусть набор положительнозначных функций 
$\psi_{1}(q), \ldots, \psi_{n}(q)$ натурального аргумента $q$ таков, что функция

$$
\psi(q)=\prod_{j=1}^{n} \psi_{j}(q)
$$

не возрастает, а ряд $\sum_{q=1}^{+\infty} \psi(q)$ расходится. Тогда для почти всех наборов $\Theta=$ $\left(\theta_{1}, \ldots, \theta_{n}\right) \in \mathbb{R}^{n}$ имеется бесконечно много натуральных чисел $q$ таких, что

$$
\left\|q \theta_{j}\right\|<\psi_{j}(q), \quad 1 \leqslant j \leqslant n .
$$

В-третьих, упомянем результат П. Галлагера из [128] (точнее, не общее утверждение, а его следствие), также касающийся случая расходимости. Этот результат гласит, что для почти всех пар вещественных чисел $\left(\xi_{1}, \xi_{2}\right)$ выполняется

$$
\liminf _{n \rightarrow \infty} n \log ^{2} n \cdot\left\|n \xi_{1}\right\|\left\|n \xi_{2}\right\|=0 .
$$

13.3. О применении метода Переса-Шлага. Здесь мы обрисуем круг задач, в которых применение метода Переса-Шлага позволяет получать нетривиальные результаты о существовании плохо приближаемых чисел.

\section{А. Приближения с субэкспоненциальными последовательностями.} Рассмотрим последовательность вещественных чисел $t_{n}, n=1,2,3, \ldots$, такую, что

$$
t_{n} \asymp \exp \left(\gamma t^{\beta}\right), \quad \gamma>0, \quad 0<\beta<1 .
$$

В [121] показано, что для любой последовательности вещественных чисел $\eta_{n}$ множество

$$
\left\{\xi \in \mathbb{R}: \inf _{n \in \mathbb{N}}\left\|t_{n} \xi-\eta_{n}\right\| \cdot t^{1-\beta} \log t>0\right\}
$$

имеет размерность Хаусдорфа равную 1 (точнее, в [121] рассмотрен только однородный случай $\eta_{n}=0$ для любого $n$; однако доказательство общего случая дословно повторяет доказательство частного случая).

Например, рассмотрим последовательность Фюрстенберга $s_{n}, n=1,2,3, \ldots$, состоящую из целых чисел вида $2^{k} \cdot 3^{m}, k, m=0,1,2,3, \ldots$, упорядоченных по возрастанию. Для нее условие (110) выполнено с $\beta=1 / 2$. Таким образом, получаем, что для всякой фиксированной последовательности неоднородностей $\eta_{n}$ множество

$$
\left\{\xi \in \mathbb{R}: \inf _{n \in \mathbb{N}}\left\|s_{n} \xi-\eta_{n}\right\| t_{n}^{1-\beta} \log t_{n}>0\right\}
$$

имеет хаусдорфову размерность 1 .

Г. Фюрстенберг [129] (простое доказательство дал М. Бошерницан [130]) доказал, что для иррационального $\xi$ множество дробных долей $\left\{2^{n} \cdot 3^{m} \alpha\right\}$ на отрезке $[0,1]$ всюду плотно и, стало быть,

$$
\liminf _{n \rightarrow \infty}\left\|s_{n} \xi\right\|=0
$$

Отметим, что о характере стремления величины $\left\|s_{n} \alpha\right\|$ к нулю замечательный результат получили недавно Ж. Бургейн, Е. Линденштраусс, П. Мишель 
и А. Венкатеш [131]. Этот результат связан с применением оценок снизу линейных форм от двух логарифмов алгебраических чисел.

В. Полиномиальные последовательности. Рассмотрим полиномиально растущую последовательность $t_{n}$, т. е. такую, что

$$
t_{n} \asymp n^{\beta}, \quad \beta>0 .
$$

В работе [122] доказано, что множество

$$
\left\{\xi \in \mathbb{R}: \inf _{n \in \mathbb{N}}\left\|t_{n} \xi-\eta_{n}\right\| t_{n} \log t_{n}>0\right\}
$$

имеет размерность Хаусдорфа $\geqslant \beta /(1+\beta)$.

Этим дан ответ на вопрос В. М. Шмидта из работы [132]. В этой же работе В. М. Шмидт задает более сложный вопрос, на который ответ до сих пор неизвестен. Мы приводим его формулировку: существуют ли такие вещественные $\xi$, для которых

$$
\inf _{n \in \mathbb{N}}\left\|n^{2} \xi\right\| n>0 ?
$$

Приведем здесь также формулировку результата А. Захареску [133] о том, что для любого иррационального $\xi$ и для любого положительного $\varepsilon$ выполнено

$$
\liminf _{n \rightarrow \infty}\left\|n^{2} \xi\right\| n^{2 / 3-\varepsilon}=0 .
$$

Результат А. Захареску является наилучшим известным на настоящее время.

Упомянутая в п. 13.2 теорема Касселса показывает, что построенные в рассматриваемых в пп. А и В задачах множества могут иметь лишь нулевую меру Лебега. Хочется также отметить, что в работе И. П. Рочева [125] имеется общая теорема о приближениях линейной формы, содержащая в себе изложенные выше результаты о существовании плохо приближаемых чисел.

Далее в текущем пункте мы остановимся на двух многомерных задачах.

С. Проблема Литтлвуда. Знаменитая гипотеза Литтлвуда предполагает, что для любых вещественных чисел $\xi_{1}, \xi_{2}$ выполняется

$$
\liminf _{n \rightarrow \infty} n\left\|n \xi_{1}\right\|\left\|n \xi_{2}\right\|=0
$$

Пользуясь случаем, автор хочет сказать, что формулировку гипотезы Литтлвуда он узнал, будучи студентом второго курса; ему рассказал о ней С. А. Довбыш.

С помощью метода Переса-Шлага можно доказать (см. [124]) существование таких $\xi_{1}, \xi_{2}$, что

$$
\liminf _{n \rightarrow \infty} n \log ^{2} n \cdot\left\|n \xi_{1}\right\|\left\|n \xi_{2}\right\|>0
$$

Из упомянутой в конце п. 13.2 теоремы П. Галлагера вытекает, что наборы $\xi_{1}, \xi_{2}$, обладающие таковым свойством, могут образовывать в $\mathbb{R}^{2}$ множество не более чем нулевой меры Лебега.

D. BAD-гипотеза. Для чисел $\alpha, \beta \in[0,1]$ при условии $\alpha+\beta=1$ и для $\delta \in(0,1 / 2)$ рассмотрим множество

$$
\operatorname{BAD}(\alpha, \beta ; \delta)=\left\{\xi=\left(\xi_{1}, \xi_{2}\right) \in[0,1]^{2}: \inf _{p \in \mathbb{N}} \max \left\{p^{\alpha}\left\|p \xi_{1}\right\|, p^{\beta}\left\|p \xi_{2}\right\|\right\} \geqslant \delta\right\} .
$$


В. М. Шмидт [63] высказал предположение о том, что для любых двух наборов $\left(\alpha_{1}, \beta_{1}\right),\left(\alpha_{2}, \beta_{2}\right) \in[0,1]^{2}, \alpha_{1}+\beta_{1}=\alpha_{2}+\beta_{2}=1$, пересечение

$$
\operatorname{BAD}\left(\alpha_{1}, \beta_{1}\right) \cap \operatorname{BAD}\left(\alpha_{2}, \beta_{2}\right)
$$

непусто. Это предположение, двойственное к гипотезе Литтлвуда, получило название BAD-гипотезы. Оно долгое время оставалось открытым. Недавно Д. Бодягин, А. Поллингтон и С. Велани получили элегантное решение этой сложной проблемы. Более того, в своей замечательной работе [134] они показали, что пересечение любого конечного (а при некоторых условиях и счетного) семейства множеств вида $\operatorname{BAD}\left(\alpha_{k}, \beta_{k}\right)$ имеет полную размерность Хаусдорфа.

Метод Переса-Шлага дает следующий результат. Для произвольной последовательности неоднородностей $\eta=\left\{\eta_{j}\right\}_{j=1}^{\infty}$ рассмотрим множества

$$
\begin{aligned}
\operatorname{BAD}_{\eta}^{*}(\alpha, \beta ; \delta)=\left\{\xi=\left(\xi_{1}, \xi_{2}\right) \in[0,1]^{2}:\right. & \\
& \left.\inf _{p \in \mathbb{N}} \max \left\{(p \log (p+1))^{\alpha}\left\|p \xi_{1}\right\|,(p \log (p+1))^{\beta}\left\|p \xi_{2}-\eta_{p}\right\|\right\} \geqslant \delta\right\} .
\end{aligned}
$$

Понятно, что

$$
\operatorname{BAD}(\alpha, \beta ; \delta) \subseteq \operatorname{BAD}_{0}^{*}(\alpha, \beta ; \delta)
$$

( $\eta=0$ здесь означает, что все $\eta_{j}$ равны нулю) и что, поскольку ряд

$$
\sum_{p=1}^{\infty} \frac{1}{p \log (p+1)}
$$

расходится, для каждой фиксированной последовательности $\eta$ объединение

$$
\operatorname{BAD}_{\eta}^{*}(\alpha, \beta)=\bigcup_{\delta>0} \operatorname{BAD}_{\eta}^{*}(\alpha, \beta ; \delta)
$$

есть множество нулевой меры Лебега.

В [123] доказано, что для произвольных $\alpha_{1}, \alpha_{2}, \beta_{1}, \beta_{2} \in[0,1], \alpha_{1}+\beta_{1}=$ $\alpha_{2}+\beta_{2}=1$, и для произвольного $0<\delta \leqslant 2^{-20}$ пересечение

$$
\operatorname{BAD}_{0}^{*}\left(\alpha_{1}, \beta_{1} ; \delta\right) \cap \operatorname{BAD}_{0}^{*}\left(\alpha_{2}, \beta_{2} ; \delta\right)
$$

непусто. Доказательство утверждения о непустоте пересечений вида

$$
\operatorname{BAD}_{\eta^{1}}^{*}\left(\alpha_{1}, \beta_{1} ; \delta\right) \cap \operatorname{BAD}_{\eta^{2}}^{*}\left(\alpha_{2}, \beta_{2} ; \delta\right)
$$

для любых двух фиксированных последовательностей $\eta^{1}$ и $\eta^{2}$ дословно повторяет доказательство соотношения (111), проведенное в [123].

Как показал Я. Бюжо в совместной работе [126], во всех перечисленных выше задачах можно доказать существование множества плохо приближаемых чисел полной размерности Хаусдорфа. Для этого следует применить принцип распределения масс (см. [135; гл. V] или [136]). Кроме этого, работа [126] содержит ряд других применений метода к задачам типа проблемы Литтлвуда. 
В настоящей статье мы не предполагаем делать сколь-нибудь подробный обзор работ, связанных с проблемами типа гипотезы Литтлвуда. Таковых работ очень много. Заметим лишь, что имеется большое количество работ, посвященных исследованиям обсуждавшихся выше вопросов с помощью теории динамических систем. Мы упомянем обзор [58].

Отметим, что в доказательствах всех сформулированных в настоящем пункте результатов о существовании плохо приближаемых чисел так или иначе присутствует следующий вариант локальной леммы Ловаса (см. [125]).

Лемма 9. Пусть $\left\{A_{n}\right\}_{n=1}^{N}$ - система событий в вероятностном пространстве $(\Omega, \mathscr{F}, \mathbf{P}),\left\{x_{n}\right\}_{n=1}^{N}-$ набор чисел из $[0,1]$. Обозначим $B_{0}=\Omega, B_{n}=$ $\bigcap_{m=1}^{n} A_{m}^{c}(1 \leqslant n \leqslant N)$, где $A_{m}^{c}=\Omega \backslash A_{m}$. Пусть для каждого $n \in\{1, \ldots, N\}$ найдется такое $m=m(n) \in\{0,1, \ldots, n-1\}$, что

$$
\mathbf{P}\left(A_{n} \cap B_{m}\right) \leqslant x_{n} \prod_{m<k<n}\left(1-x_{k}\right) \cdot \mathbf{P}\left(B_{m}\right)
$$

(если $m=n-1$, то считаем $\left.\prod_{m<k<n}\left(1-x_{k}\right)=1\right)$. Тогда при $1 \leqslant n \leqslant N$ справедливо неравенство

$$
\mathbf{P}\left(B_{n}\right) \geqslant\left(1-x_{n}\right) \mathbf{P}\left(B_{n-1}\right) .
$$

13.4. Об $(\alpha, \beta)$-играх. Напомним определение $(\alpha, \beta)$-игры Банаха-Мазура-Шмидта в пространстве $\mathbb{R}^{d}$. Итак, пусть $\alpha, \beta \in(0,1)$, и пусть $S \subseteq \mathbb{R}^{d}-$ некоторое множество. Играют белые и черные. Сначала черные выбирают замкнутый шар (в sup-норме) $B_{1}$ диаметра $l\left(B_{1}\right)=2 \rho$. Затем белые выбирают замкнутый шар $W_{1} \subset B_{1}$ диаметра $l\left(W_{1}\right)=\alpha l\left(B_{1}\right)$. Потом черные выбирают замкнутый шар $B_{2} \subset W_{1}$ диаметра $l\left(B_{2}\right)=\beta l\left(W_{1}\right)$, и т. д. В результате образуется последовательность вложенных замкнутых шаров $B_{1} \supset W_{1} \supset B_{2} \supset$ $W_{2} \supset \cdots$ с диаметрами $l\left(B_{i}\right)=2(\alpha \beta)^{i-1} \rho$ и $l\left(W_{i}\right)=2 \alpha(\alpha \beta)^{i-1} \rho(i=1,2, \ldots)$. Очевидно, что множество $\bigcap_{i=1}^{\infty} B_{i}=\bigcap_{i=1}^{\infty} W_{i}$ состоит из одной-единственной точки. Если $\bigcap_{i=1}^{\infty} B_{i} \in S$, то говорят, что белые выиграли игру. Множество $S$ называется $(\alpha, \beta)$-выигрышным, если белые могут выиграть игру независимо от того, как играют черные. Множество $S$ называется $\alpha$-выигрышным, если оно является $(\alpha, \beta)$-выигрышным при каждом $\beta \in(0,1)$. Выигрышной размерностью windim $\mathscr{A}$ множества $\mathscr{A} \in \mathbb{R}$ называется супремум тех $\alpha$, при которых множество $\mathscr{A}$ является $\alpha$-выигрышным. Если windim $\mathscr{A}>1 / 2$, то, как легко видеть, $\mathscr{A}=\mathbb{R}^{d}$. При $0<\alpha \leqslant 1 / 2$ существуют нетривиальные $\alpha$-выигрышные множества. В. М. Шмидт [20], [24] доказал ряд общих теорем о выигрышных множествах. Ниже мы приводим две из них.

ТеОрема 70. Если $\alpha>0$, то всякое $\alpha$-выигрышное множество в $\mathbb{R}^{d}$ имеет размерность Хаусдорфа равную $d$.

Теорема 71. Если $\alpha>0$, то пересечение любого счетного числа $\alpha$-выигрышных множеств снова будет $\alpha$-выигрышным. 
Далее мы приводим ряд примеров выигрышных множеств.

А. Следующий результат имеется у В. М. Шмидта [20].

Теорема 72. Пусть $d=1$. Забиксируем натуральное $q \geqslant 2$. Множество

$$
\mathscr{N}_{q}=\left\{x \in \mathbb{R}: \exists C\left(x>0 \forall n \in \mathbb{N}\left\|q^{n} x\right\| \geqslant C(x)\right\},\right.
$$

состоящее из вещественных чисел, не являющихся нормальными по натуральному основанию $q \geqslant 2$, имеет выигрышную размерность $1 / 2$.

В. При $d=1$ рассмотрим лакунарную последовательность положительных чисел $t_{j}$ (определение лакунарности см. в п. 13.1). Обобщая рассуждения В. М. Шмидта, легко видеть, что множество

$$
\mathscr{N}=\left\{x \in \mathbb{R}: \exists C(x)>0 \forall \in \mathbb{N}\left\|t_{n} x\right\| \geqslant C(x)\right\}
$$

имеет выигрышную размерность $1 / 2$.

С. Для последовательностей сублакунарного роста автор [137] доказал следующий результат.

ТеОрема 73. Пусть последовательность положительных чисел $t_{n}$ такова, чmo

$$
\forall \varepsilon>0 \exists N_{0} \forall n \geqslant N_{0}: \frac{t_{n+1}}{t_{n}} \geqslant 1+\frac{1}{n^{\varepsilon}} .
$$

Тогда при каждом положительном $\delta$ множество

$$
\mathscr{A}_{\delta}=\left\{x \in \mathbb{R}: \exists c(x)>0 \forall n \in \mathbb{N}\left\|t_{n} x\right\|>c(x) / n^{\delta}\right\}
$$

будет $\alpha$-выигрышным с любым заданным наперед $\alpha \in(0,1 / 2)$, и, таким образом, windim $\mathscr{A}_{\delta}=1 / 2$.

D. Особо следует поговорить о плохо приближаемых системах линейных форм. Рассматриваемый в настоящей статье набор вещественных чисел $\Theta=$ $\left\{\theta_{j}^{i}, 1 \leqslant i \leqslant m, 1 \leqslant j \leqslant n\right\}$ называется плохо приближаемым, если найдется положительная постоянная $\gamma$ такая, что для соответствующей системы линейных форм $\mathbf{L}_{\Theta}(\mathbf{x})=\left\{L_{j}(\mathbf{x}), 1 \leqslant j \leqslant n\right\}$ будет выполнено

$$
\max _{1 \leqslant j \leqslant n}\left\|L_{j}(\mathbf{x})\right\| \geqslant \gamma \cdot\left(\max _{1 \leqslant i \leqslant m}\left|x_{i}\right|\right)^{-m / n} \forall \mathbf{x} \in \mathbb{R}^{m} \backslash\{\mathbf{0}\} .
$$

Хорошо известно, что плохо приближаемые наборы $\Theta$ существуют и что при всех $n, m$ множество всех плохо приближаемых наборов $\Theta$ есть множество нулевой меры Лебега в $\mathbb{R}^{n m}$ (последнее вытекает, например, из метрической теоремы А. Я. Хинчина, упомянутой в п. 13.2).

С другой стороны, как легко видеть, набор $\Theta$ будет плохо приближаем в том и только том случае, когда для функции Ярника (20) выполняется

$$
\liminf _{t \rightarrow+\infty} t^{m / n} \psi_{\Theta}(t)>0 .
$$

Ясно, что плохо приближаемый набор $\Theta$ образует регулярную систему (в терминологии А. Я. Хинчина).

Сформулируем теорему В. М. Шмидта из [138]. 
ТеОРема 74. При каждом значении размерностей $n, m$ множество плохо приближаемых наборов $\Theta$ в $\mathbb{R}^{d}, d=n m$, имеет выигрышную размерность $1 / 2$.

Отметим, что плохо приближаемым системам чисел посвящено огромное количество литературы, обзор которой мы просто не в состоянии сделать.

Е. Отметим в заключение, что о выигрышных множествах имеется довольно много других работ (см., например, [51], [52], [56], [139]-[145]).

\section{Список литературы}

[1] A. Khintchine, "Über eine Klasse linearer diophantischer Approximationen", Rendiconti Circ. Math. Palermo, 50:2 (1926), 170-195.

[2] A. Khintchine, "Zwei Bemerkungen zu einer Arbeit des Herrn Perron", Math. Z., 22:1 (1925), 274-284.

[3] A. Khintchine, "Zur metrischen Theorie der diophantischen Approximationen", Math. Z., 24:1 (1926), 706-714.

[4] A. Khintchine, "Über die angenäherte Auflösung linearer Gleichungen in ganzen Zahlen", Acta Arith., 2 (1937), 161-172.

[5] А. Я. Хинчин, "О задаче Чебышёва", Изв. АН СССР. Сер. матем., 10:4 (1946), $281-294$.

[6] А.Я. Хинчин, "Теорема переноса для сингулярных систем линейных уравнений”, Докл. АН СССР, 59:2 (1948), 217-218.

[7] А.Я. Хинчин, "Регулярные системы линейных уравнений и общая задача Чебышёва", Изв. АН СССР. Сер. матем., 12:3 (1948), 249-258.

[8] А.Я. Хинчин, "О некоторых приложениях метода добавочной переменной", УМH, 3:6 (1948), 188-200.

[9] V. Jarník, "Zum Khintchineschen "Übertragungssatz", Acad. Sci. URSS, 3, Trav. Inst. math., Tbilissi, 1938, 193-216.

[10] V. Jarník, "Remarques à l'article précédent de M. Mahler", Časopis Mat. Fysik., 68 (1939), 103-111.

[11] V. Jarník, "On linear inhomogeneous Diophantine approximations", Rozpravy II. Třidy České Akad., 51:29 (1941), 21 pp.

[12] V. Jarník, "Sur les approximations diophantiques linéaires non homogènes", Acad. Tchéque Sci. Bull. Int. Cl. Sci. Math. Nat., 47:16 (1950), 145-160.

[13] В. Ярник, "К теории однородных линейных диофантовых приближений", Чехослов. матем. журн., 4(79) (1954), 330-353.

[14] V. Jarník, "Eine Bemerkung über diophantische Approximationen", Math. Z., 72:1 (1959), 187-191.

[15] V. Jarník, "Eine Bemerkung zum 'Übertragungssatz"', Bulgar. Akad. Nauk Izv. Mat. Inst., 3:2 (1959), 169-175.

[16] А.Я. Хинчин, Избранные труды по теории чисел, МЦНМО, М., 2006, ISBN: 5-94057-088-7, 260 c.

[17] В. В. Козлов, "Интегрируемость и неинтегрируемость в гамильтоновой механике", УМН, 38:1 (1983), 3-67; англ. пер.: V. V. Kozlov, "Integrability and nonintegrability in Hamiltonian mechanics", Russian Math. Surveys, 38:1 (1983), 1-76.

[18] В.В. Козлов, Н.Г. Мощевитин, "О диффузии в гамильтоновых системах", Вестн. Моск. ун-та. Сер. 1. Матем., мех., 1997, №5, 49-52; англ. пер.: V. V. Kozlov, N. G. Moshchevitin, "On diffusion in Hamiltonian systems", Mosc. Univ. Mech. Bull., 52:5 (1997), 18-22.

[19] Y. Peres, W. Schlag, "Two Erdös problems on lacunary sequences: chromatic numbers and Diophantine approximations", Bull. London Math. Soc., 42:2 (2010), 295-300; arXiv: 0706.0223v1. 
[20] W. M. Schmidt, "On badly approximable numbers and certain games", Trans. Amer. Math. Soc., 123:1 (1966), 178-199.

[21] M. Waldschmidt, Report on some recent advances in Diophantine approximation, arXiv: 0908.3973v1.

[22] J. F. Koksma, Diophantische Approximationen, Ergeb. Math. Grenzgeb., 4, Springer, Berlin, 1936, 157 pp.

[23] Дж.В.С. Касселс, Введение в теорию диофантовых приближений, ИЛ, М., 1961, 213 с.; пер. с англ.: J. W. S. Cassels, An introduction to Diophantine approximation, Cambridge Tracts Math. Math. Phys., 45, Cambridge Univ. Press, New York, 1957, 166 pp.

[24] В. Шмидт, Диофантовы приближения, Мир, М., 1983, 230 с.; пер. с англ.: W. M. Schmidt, Diophantine approximation, Lecture Notes in Math., 785, Springer, Berlin, 1980, ISBN: 3-540-09762-7, 299 pp.

[25] H. Davenport, W. M. Schmidt, "Dirichlet's theorem on Diophantine approximation. II", Acta Arith., 16 (1970), 413-424.

[26] C. Chabauty, E. Lutz, "Sur les approximations diophantiennes linéaires réelles. I. Problème homogène", C.R. Acad. Sci. Paris, 231 (1950), 887-888.

[27] J. Lesca, "Sur un résultat de Jarník", Acta Arith., 11 (1966), 359-364.

[28] J. Lesca, "Existence de systèmes $p$-adiques admettant une approximation donnée", Acta Arith., 11 (1966), 365-370.

[29] A. Apfelbeck, "A contribution to Khintchine's principle of transfer", Czech. Math. J., 1(76) (1952), 119-147.

[30] J.S. Lagarias, "Best simultaneous Diophantine approximation. II: Behavior of consecutive best approximations", Pacific J. Math., 102:1 (1982), 61-88.

[31] H. Davenport, W. M. Schmidt, "Approximation to real numbers by quadratic irrationals", Acta Arith., 13 (1967), 169-176.

[32] Н. Г. Мощевитин, "О наилучших совместных приближениях", УМH, 51:6 (1996), 213-214; англ. пер.: N. G. Moshchevitin, "On best simultaneous approximations", Russian Math. Surveys, 51:6 (1996), 1214-1215.

[33] N. G. Moshchevitin, "Best Diophantine approximations: the phenomenon of degenerate dimension", Surveys in geometry and number theory: reports on contemporary Russian mathematics, London Math. Soc. Lecture Note Ser., 338, Cambridge Univ. Press, Cambridge, 2007, 158-182.

[34] Н. Г. Мощевитин, О. Н. Герман, "Теорема о наилучших диофантовых приближениях для линейной формы", Механико-математический факулътет МГУ им. M. В. Ломоносова, Сборник статей, посвященный 70-летию академика В. А. Садовничего, Современные проблемы математики и механики, 4, № 3, МГУ, М., 2009, 130-135; arXiv: 0812.2455v1.

[35] Н. Г. Мощевитин, "О геометрии наилучших приближений", Докл. РАН, 359:5 (1998), 587-589; англ. пер.: N. G. Moshchevitin, "On the geometry of best approximations", Dokl. Math., 57:2 (1998), 261-263.

[36]А.Я. Хинчин, Цепные дроби, Физматгиз, М., 1961, 112 с.; англ. пер.: А. Ya. Khinchin, Continued fractions, Univ. Chicago Press, Chicago and London, 1964, xi, 95 pp.

[37] T.W. Cusick, M.E. Flahive, The Markoff and Lagrange spectra, Math. Surveys Monogr., 30, Amer. Math. Soc., Providence, RI, 1989, ISBN: 0-8218-1531-8, ix, $97 \mathrm{pp}$.

[38] А.В. Малышев, "Спектры Маркова и Лагранжа (обзор литературы)", Записки науч. сем. ЛОМИ, 67 (1977), 5-38; англ. пер.: A. V. Malyshev, "Markov and Lagrange spectra (survey of the literature)", J. Math. Sci., 16:1 (1981), 767-788.

[39] H. Davenport, W. M. Schmidt, "Dirichlet's theorem on Diophantine approximation", Simposia Mathematica, v. IV (INDAM, Rome, 1968/69), Academic Press, London, 1970, 113-132. 
[40] В. А. Иванов, "О начале луча в спектре Дирихле одной задачи теории диофантовых приближений”, Записки науч. сем. ЛОМИ, 93 (1980), 164-185; англ. пер.: V. A. Ivanov, "Origin of the ray in the Dirichlet spectrum of a problem in the theory of Diophantine approximations", J. Math. Sci., 19:2 (1982), 1169-1183.

[41] I. D. Kan, N. G. Moshchevitin, "Approximation to two real numbers", Uniform Distribution Theory, 5:2 (2010), 79-86; arXiv: 0910.2428.

[42] Н.Г. Мощевитин, "Наилучшие совместные приближения: нормы, сигнатуры и асимптотические направления", Матем. заметки, 67:5 (2000), 730-737; англ. пер.: N. G. Moshchevitin, "Best simultaneous approximations: Norms, signatures, and asymptotic directions", Math. Notes, 67:5 (2000), 618-624.

[43] M. Laurent, "Exponents of Diophantine approximation in dimension two", Canad. J. Math., 61:1 (2009), 165-189; arXiv: math/0611352v1.

[44] N. G. Moshchevitin, Contribution to Vojtech Jarnik, arXiv: 0912.2442v2.

[45] J. W. S. Cassels, "Über $\underline{\lim } x|x \theta+\alpha-y|$ ", Math. Ann., 127:1 (1954), 288-304.

[46] E. S. Barnes, "On linear inhomogeneous Diophantine approximations", J. London Math. Soc., 31:1 (1956), 73-79.

[47] T. W. Cusick, W. Moran, A. D. Pollington, "Hall's ray in inhomogeneous Diophantine appproximations", J. Austral. Math. Soc. Ser. A, 60 (1996), 42-50.

[48] H. J. Godwin, "On the theorem of Khintchine", Proc. London Math. Soc. (3), 3:1 (1953), 211-221.

[49] D. Kleinbock, "Badly approximable systems of affine forms", J. Number Theory, 79:1 (1999), 83-102.

[50] Y. Bugeaud, S. Harrap, S. Kristensen, S. Velani, On shrinking targets for $\mathbb{Z}$ actions on tori, arXiv: $0807.3863 v 1$.

[51] J. Tseng, "Badly approhimable affine forms and Schmidt games", J. Number Theory, 129:12 (2009), 3020-3025.

[52] M. Einsiedler, J. Tseng, Badly approximable systems of affine forms, fractals, and Schmidt games, arXiv: 0912.2445.

[53] N. G. Moshchevitin, "A note on badly approximable affine forms and winning sets", arXiv: 0812.39998.

[54] D. Y. Kleinbock, G. A. Margulis, "Flows on homogeneous spaces and Diophantine approximation on manifolds", Ann. of Math. (2), 148:1 (1998), 339-360; arXiv: math/9810036.

[55] D. Kleinbock, G. Margulis, J. Wang, Metric Diophantine approximations for systems of linear forms via dynamics, arXiv: 0904.2795v1.

[56] D. Kleinbock, B. Weiss, "Modified Schmidt games and Diophantine approximation with weights", Adv. Math., 223:4 (2010), 1276-1298; arXiv: 0805.2934v1.

[57] D. Kleinbock, B. Weiss, "Dirichlet's theorem on Diophantine approximations and homogeneous flows", J. Mod. Dyn., 2:1 (2008), 43-62.

[58] A. Gorodnik, "Open problem in dynamics and related fields", J. Mod. Dyn., 1:1 (2007), 1-35.

[59] W. M. Schmidt, "Diophantine approximations and certain properties of lattices", Acta Arith., 18 (1971), 195-178.

[60] K. Mahler, "Lattice points in two-dimensional star domains. I", Proc. London Math. Soc. (2), 49:1 (1946), 128-157; "Lattice points in two-dimensional star domains. II", 158-167; "Lattice points in two-dimensional star domains. III", 168-183.

[61] K. Mahler, "On lattice points in $n$-dimensional star bodies. I. Existence theorems", Proc. Roy. Soc. London. Ser. A., 187 (1946), 151-187; "II. Reducibility theorems", Proc. Nederl. Akad. Wetensch., 49 (1946), 331-343, 444-454, 526-532, 622-631.

[62] Дж. В.С. Касселс, Введение в геометрию чисел, Мир, М., 1965, 421 с.; пер. с англ.: J. W.S. Cassels, An introduction to the geometry of numbers, Grundlehren Math. Wiss., 99, Springer-Verlag, Berlin-Göttingen-Heidelberg, 1959, 344 pp. 
[63] W.M. Schmidt, "Open problems in Diophantine approximations", Diophantine approximations and transcendental numbers (Luminy, 1982), Progr. Math., 31, Birkhäuser, Boston, MA, 1983, 271-289.

[64] W. M. Schmidt, L. Summerer, "Parametric geometry of numbers and applications", Acta Arith., 140:1 (2009), 67-91.

[65] N. G. Moshchevitin, Proof of W. M. Schmidt conjecture concerning successive minima of a lattice, arXiv: 0804.0120v1.

[66] F. J. Dyson, "On simultaneous Diophantine approximations", Proc. London Math. Soc. (2), 49:1 (1947), 409-420.

[67] K. Mahler, "On compound convex bodies. I", Proc. London Math. Soc. (3), 5 (1955), 358-379; "On compound convex bodies. II", 380-384.

[68] M. Laurent, "On transfer inequalities in Diophantine approximation", Analytic number theory, Cambridge Univ. Press, Cambridge, 2009, 306-314; arXiv: math/ $0703146 v 1$.

[69] Y. Bugeaud, M. Laurent, "On transfer inequalities in Diophantine approximation. II", Math. Z., 265:2 (2010), 249-262; arXiv: 0811.2102v1.

[70] Н. М. Коробов, "О некоторых вопросах теории диофантовых приближений", УМH, 22:3 (1967), 83-118; англ. пер.: N. M. Korobov, "Some problems in the theory of Diophantine approximation", Russian Math. Surveys, 22:3 (1967), 80-118.

[71] Н. М. Коробов, "Об одной оценке А. О. Гельфонда", Вестн. Моск. ун-та. Сер. 1. Maтем., мех., 1983, №3, 3-7; англ. пер.: N. M. Korobov, "An estimate of A. O. Gelfond", Moscow Univ. Math. Bull., 38:3 (1983), 1-6.

[72] А. О. Гельфонд, "О теореме Минковского для линейных форм и теоремах переноса", приложение к кн.: Дж.В.С. Касселс, Введение в теорию диофантовых приближений, ИЛ, М., 1961, 202-209.

[73] Ю. В. Каширский, "Теоремы переноса”, Докл. АН СССР, 149 (1963), 1019-1022; англ. пер.: Yu. V. Kashirskii, "Transference theorems", Soviet Math. Dokl., 4 (1963), $505-508$.

[74] C. L. Siegel, "Neuer Beweis des Satzes von Minkowski über lineare Formen", Math. Ann., 87:1-2 (1922), 36-38.

[75] W. M. Schmidt, Y. Wang, "A note on a tranference theorem of linear forms", Sci. Sinica, 22:3 (1979), 276-280.

[76] W. M. Schmidt, "On heights of algebraic subspaces and Diophantine approximations", Ann. of Math. (2), 85:3 (1967), 430-472.

[77] Y. Bugeaud, M. Laurent, "On exponents of homogeneous and inhomogeneous Diophantine approximation", Moscow Math. J., 5:4 (2005), 747-766.

[78] Y. Cheung, Hausdorff dimension of the set of singular pairs, arXiv: 0709.4534v2.

[79] R. C. Baker, "Singular $n$-tuples and Hausdorff dimension. II", Math. Proc. Cambridge Philos. Soc., 111:3 (1992), 577-584.

[80] R. C. Baker, "Singular n-tuples and Hausdorff dimension", Math. Proc. Cambridge Philos. Soc., 81:3 (1977), 377-385.

[81] К. Ю. Явид, "Оценка размерности Хаусдорфа множеств сингулярных векторов”, Докл. АН БССР, 31:9 (1987), 777-780.

[82] B.P. Rynne, "A lower bound for the Hausdorff dimension of sets of singular n-tuples", Math. Proc. Cambridge Philos. Soc., 107:2 (1990), 387-394.

[83] B. P. Rynne, "The Hausdorff dimension of certain sets of singular $n$-tuples", Math. Proc. Cambridge Philos. Soc., 108:1 (1990), 105-110.

[84] M. M. Dodson, "Hausdorff dimension, lower order and Khintchine's theorem in metric Diophantine approximations", J. Reine Angew. Math., 432 (1992), 69-76.

[85] В. И. Берник, Ю. В. Мельничук, Диофантовы приближения и размерность Хаусдорфа, Наука и техника, Минск, 1988, ISBN: 5-343-00231-5, 144 с. 
[86] V.I. Bernik, M. M. Dodson, Metric Diophantine approximations on manifolds, Cambridge Tracts in Math., 137, Cambridge Univ. Press, Cambridge, 1999, ISBN: 0-521-43275-8, $172 \mathrm{pp}$.

[87] W. M. Schmidt, "Two questions in Diophantine approximation", Monatsh. Math., 82:3 (1976), 237-245.

[88] P. Thurnheer, "Zur diophantischen Approximation von zwei reellen Zahlen", Acta Arith., 44:3 (1984), 201-206.

[89] P. Thurnheer, "On Dirichlet's theorem concerning Diophantine approximation", Acta Arith., 54:3 (1990), 241-250.

[90] Y. Bugeaud, S. Kristensen, "Diophantine exponents for mildly restricted approximation", Ark. Mat., 47:2 (2009), 243-266.

[91] N. G. Moshchevitin, On Diophantine approximations with positive integers: a remark to W. M. Schmidt's theorem, arXiv: 0904.1906v1.

[92] H. Davenport, W. M. Schmidt, "A theorem on linear forms", Acta Arith., 14 (1968), 209-223.

[93] Г. Вейль, "О равномерном распределении чисел по модулю один”, Избраннъе труды, Наука, М., 1984, 58-93; пер. с нем.: H. Weyl, "Über die Gleichverteilung von Zahlen mod. Eins", Math. Ann., 77 (1916), 313-352.

[94] И.П. Корнфельд, Я.Г. Синай, С.В. Фомин, Эргодическая теория, Наука, M., 1980, 384 с.; англ. пер.: I. P. Cornfeld, S. V. Fomin, Ya. G. Sinai, Ergodic theory, Grundlehren Math. Wiss., 245, Springer-Verlag, New York, 1982, ISBN: 0-387-90580-4, x+486 pp.

[95] Yu. Peres, "A combinatorial application of the maximal ergodic theorem", Bull. London Math. Soc., 20:3 (1988), 248-252.

[96] П. Боль, "Об одном дифференциальном уравнении из теории возмущений": П. Боль, Избранные труды, Изд-во АН Латв. ССР, Рига, 1961, 127-154; пер. с англ.: P. Bohl, "On a differential equation from perturbation theory", J. Reine Angew. Math., 131 (1906), 268-321.

[97] В. В. Козлов, “Финальные свойства интегралов от квазипериодических функций”, Вестн. Моск. ун-та. Сер. 1. Матем., мех., 1978, № 1, 106-115; англ. пер.: V. V. Kozlov, "On integrals of quasiperiodic functions", Mosc. Univ. Mech. Bull., 33:1-2 (1978), 31-38.

[98] В. В. Козлов, Методи качественного анализа в динамике твердого тела, МГУ, M., 1980, 231 c.

[99] G. Halász, "Remarks on the remainder in Birkhoff's ergodic theorem", Acta Math. Acad. Sci. Hungar., 28:3-4 (1976), 389-395.

[100] Е. А. Сидоров, "Об условиях равномерной устойчивости по Пуассону цилиндрических систем", УМН, 34:6 (1979), 184-188; англ. пер.: Е. A. Sidorov, "Conditions for uniform Poisson stability of cylindrical systems", Russian Math. Surveys, 34:6 (1979), 220-224.

[101] С. В. Конягин, "О возвращаемости интеграла нечетной условнопериодической функции", Матем. заметки, 61:4 (1997), 570-577; англ. пер.: S. V. Konyagin, "Recurrence of the integral of an odd conditionally periodic function", Math. Notes, 61:4 (1997), 473-479.

[102] Н. Г. Мощевитин, “О возвращаемости интеграла гладкой условнопериодической функции", Матем. заметки, 63:5 (1998), 737-748; англ. пер.: N. G. Moshchevitin, "Recurrence of the integral of a smooth conditionally periodic function", Math. Notes, 63:5 (1998), 648-657.

[103] N. G. Moshchevitin, "Distribution of Kronecker sequence", Algebraic number theory and Diophantine analysis (Graz, 1998), de Gruyter, Berlin, 2000, 311-329.

[104] Н. Г. Мощевитин, Вопросы возвращаемости динамических систем и диофантовы приближения, Дис. ... докт. физ.-матем. наук, М., 2003. 
[105] Н.Г. Мощевитин, "О невозвращаемости интеграла условно-периодической функции", Матем. заметки, 49:6 (1991), 138-140; англ. пер.: N. G. Moshchevitin, "Nonrecursiveness of the integral of a conditionally periodic function", Math. Notes, 49:6 (1991), 650-652.

[106] H. Poincaré, "Sur les séries trigonométriques", Comptes Rendus, 101:2 (1886), 1131-1134.

[107] А. Пуанкаре, О кривых, определяемых дифференииальными уравнениями, Гостехиздат, М., Л., 1947.

[108] Н. Г. Мощевитин, “О возвращаемости интеграла гладкой трехчастотной условнопериодической функции”, Матем. заметки, 58:5 (1995), 723-735; англ. пер.: N. G. Moshchevitin, "Recurrence of the integral of a smooth three-frequency conditionally periodic function", Math. Notes, 58:5 (1995), 1187-1196.

[109] Н. Г. Мощевитин, "Распределение значений линейных функций и асимптотическое поведение траекторий некоторых динамических систем", Maтем. заметки, 58:3 (1995), 394-410; англ. пер.: N. G. Moshchevitin, "Distribution of values of linear functions and asymptotic behavior of trajectories of some dynamical systems", Math. Notes, 58:3 (1995), 948-959.

[110] Е.В. Коломейкина, Н.Г. Мощевитин, "О невозвращаемости в среднем сумм вдоль последовательности Кронекера", Матем. заметки, 73:1 (2003), 140-143; англ. пер.: E. V. Kolomeikina, N. G. Moshchevitin, "Nonrecurrence in mean of sums along the Kronecker sequence", Math. Notes, 73:1-2 (2003), 132-135.

[111] O. N. German, N. G. Moshchevitin, "Linear forms of a given Diophantine type", J. Number Theory Bordeaux (to appear); arXiv: 0812.4896v3.

[112] О.Н. Герман, “Асимптотические направления для наилучших приближений $n$-мерной линейной формы", Матем. заметки, 75:1 (2004), 55-70; англ. пер.: O.N. German, "Asymptotic directions for best approximations of $n$-dimensional linear forms", Math. Notes, 75:1-2 (2004), 51-65.

[113] P. Erdös, "Problems and results on Diophantine approximations. II", Répartition modulo 1 (Marseille-Luminy, 1974), Lecture Notes in Math., 475, Springer, Berlin, 1975, 89-99.

[114] A. D. Pollington, "On the density of sequence $\left\{n_{k} \theta\right\} "$, Illinois J. Math., 23:4 (1979), $511-515$.

[115] D. de Mathan, "Numbers contravening a condition in density modulo 1", Acta Math. Acad. Sci. Hungar., 36:3-4 (1980), 237-241.

[116] Y. Katznelson, "Chromatic numbers of Cayley graphs on $\mathbb{Z}$ and recurrence", Combinatorica, 21:2 (2001), 211-219.

[117] Р. К. Ахунжанов, Н. Г. Мощевитин, "О распределении по модулю 1 субэкспоненциальных последовательностей”, Матем. заметки, 77:6 (2005), 803-813; англ. пер.: R. K. Akhunzhanov, N. G. Moshchevitin, "Density modulo 1 of sublacunary sequences", Math. Notes, 77:5-6 (2005), 741-750.

[118] A. Dubickas, "On the fractional parts of lacunary sequences", Math. Scand., 99:1 (2006), 136-146.

[119] A. Dubickas, "An approximation by lacunary sequence of vectors", Combin. Probab. Comput., 17:3 (2008), 339-345.

[120] N. G. Moshchevitin, A version of the proof for Peres-Schlag's theorem on lacunary sequences, arXiv: $0708.2087 \mathrm{v} 2$.

[121] N. G. Moshchevitin, Density modulo 1 of sublacunary sequences: application of Peres-Schlag's arguments, arXiv: 0709.3419v2.

[122] N. G. Moshchevitin, "On small fractional parts of polynomials", J. Number Theory, 129:2 (2009), 349-357.

[123] N. G. Moshchevitin, "On simultaneously badly approximable numbers", Bull. London Math. Soc., 42:1 (2010), 149-154. 
[124] N. G. Moshchevitin, Badly approximable numbers related to the Littlewood conjecture, arXiv: 0810.0777.

[125] I. Rochev, On distribution of fractional parts of linear forms, arXiv: 0811.1547v1.

[126] Y. Bugeaud, N. Moshchevitin, Badly approximable numbers and Littlewood-type problems, arXiv: 0905.0830.

[127] J. W. S. Cassels, "Some metrical theorems in Diophantine approximation. I", Proc. Cambridge Philos. Soc., 46 (1950), 209-218.

[128] P. Gallagher, "Metric simultaneous Diophantine approximation", J. London Math. Soc., 37:1 (1962), 387-390.

[129] H. Furstenberg, "Disjointness in ergodic theory, minimal sets, and a problem in Diophantine approximation", Math. Systems Theory, 1:1 (1967), 1-49.

[130] M. D. Boshernitzan, "Elementary proof of Furstenberg's Diophantine result", Proc. Amer. Math. Soc., 122:1 (1994), 67-70.

[131] J. Bourgain, E. Lindenstrauss, P. Michel, A. Venkatesh, "Some effective results for $\times a \times b$ ", Ergodic Theory Dynam. Systems, 29:6 (2009), 1705-1722.

[132] W. M. Schmidt, Small fractional parts of polynomials, Regional Conf. Ser. in Math., 32, Amer. Math. Soc., Providence, RI, 1977, ISBN: 0-8218-1682-9, 41 pp.

[133] A. Zaharescu, "Small values of $n^{2} \alpha(\bmod 1)$ ", Invent. Math., 121:2 (1995), 379-388.

[134] D. Badziahin, A. Pollington, S. Velani, On a problem in simultaneous Diophantine approximation: Schmidt's conjecture, arXiv: 1001.2694v1.

[135] Y. Bugeaud, Approximation by algebraic numbers, Cambridge Tracts in Math., 160, Cambridge Univ. Press, Cambridge, 2004, ISBN: 0-521-82329-3.

[136] K. Falconer, Fractal geometry: Mathematical foundations and applications, Wiley, Chichester, 1990, ISBN: 0-471-92287-0, 288 pp.

[137] Н. Г. Мощевитин, "О сублакунарных последовательностях и выигрышных множествах", Матем. заметки, 78:4 (2005), 634-637; англ. пер.: N. G. Moshchevitin, "Sublacunary sequences and winning sets", Math. Notes, 78:3-4 (2005), 592-596.

[138] W. M. Schmidt, "Badly approximable systems of linear forms", J. Number Theory, 1:2 (1969), 139-154.

[139] Р.К. Ахунжанов, "Об анормальных числах", Матем. заметки, 72:1 (2002), 150-152; англ. пер.: R. K. Akhunzhanov, "On nonnormal numbers", Math. Notes, 72:1-2 (2002), 135-137.

[140] Р. К. Ахунжанов, "О распределении по модулю 1 экспоненциальных последовательностей”, Матем. заметки, 76:2 (2004), 163-171; англ. пер.: R. K. Akhunzhanov, "On the distribution modulo 1 of exponential sequences", Math. Notes, 76:1-2 (2004), 153-160.

[141] Y. Bugeaud, R. Broderick, L. Fishman, D. Kleinbock, B. Weiss, Schmidt games, fractals, and numbers normal to no base, arXiv: 0909.4251.

[142] В.А. Дремов, "Об областях ( $\alpha, \beta)$-выигрышности", Докл. PAH, 384:2 (2002), 304-307; англ. пер.: V. A. Dremov, "On domains of $(\alpha, \beta)$-winning", Dokl. Math., 65:3 (2002), 365-368.

[143] L. Fishman, "Schmidt's game, badly approximable matrices and fractals", J. Number Theory, 129:9 (2009), 2133-2153.

[144] C. Freiling, "An answer to a question of Schmidt on $(\alpha, \beta)$ games", J. Number Theory, 15:2 (1982), 226-228.

[145] C. Freiling, "Some new games and badly approximable numbers", J. Number Theory, 19:2 (1984), 195-202.

Н. Г. Мощевитин (N. G. Moshchevitin)

Поступила в редакцию Московский государственный университет 29.03.2010 им. М. В. Ломоносова

E-mail: moshchevitin@rambler.ru 